\title{
Forensic Lung Pathology
}

\author{
Michael A. Graham
}

Sudden and unexpected natural deaths and nonnatural deaths may result from various pulmonary conditions. Additionally, several nonpulmonary conditions of forensic significance may be complicated by the development of respiratory lesions. Certain situations with pulmonary pathology are particularly likely to be critically scrutinized and may form the basis of allegations of medical negligence, other personal injury liability, or wrongful death. ${ }^{1}$

The forensic evaluation of lethal or nonlethal conditions is essentially the same and conceptually differs little from a clinician's approach to a patient with a similar condition (Table 31.1). In some cases this diagnostic/investigative process is quite broad, whereas in other cases the issues are of very limited scope and the investigation may be narrowly focused. The pathologist may play a critical role in these evaluations, not only by addressing the abovenoted issues, but also by documenting pertinent findings that other experts may rely on in formulating their opinions; properly recognizing, collecting, preserving, and transmitting physical evidence; and presenting findings and opinions in appropriate legal forums.

The focus of many forensic evaluations is on establishing the cause and manner of death. The elucidation of the mechanism of death may also be of importance in some cases. In other cases, the cause of disease or injury, timing of lesions, establishing the presence or extent of lesions and any response to therapy, evaluating modifying or contributory factors to death or disability, or correlating findings with antemortem activity or condition may be at issue. As noted above, the process to arrive at these determinations has the same components as a clinician's approach to a patient: assessment of the history, physical examination, and ancillary studies.

In fatalities, determinations of the cause of death, manner of death, and mechanism of death are often important. Commonly, there is confusion about the meanings and use of these terms as they apply to medicolegal death investigation and death certification.
The cause of death is the disease or injury that begins the unbroken pathophysiologic sequence leading to death. The disease/injury that causes death at a particular time is often referred to as the immediate cause of death. The disease/injury that starts the unbroken chain of medical events culminating in death is referred to as the underlying cause of death. Proper recognition of the latter is very important for death certification, epidemiology, public policy, and the proper resolution of criminal justice and civil liability issues. ${ }^{2}$ The mechanism of death is the nonspecific pathophysiologic alteration through which the cause of death exerts its lethal influence. The manner of death is a term peculiar to death certification that describes how death came about - through natural causes, homicide, suicide, or accident. ${ }^{2}$ The determination of the manner of death is heavily dependent on the investigation of the circumstances surrounding the death or, if death is delayed, the illness or injury ultimately responsible for death. In some cases the information will not enable a clear determination to be made, and a classification of undetermined may be warranted.

The most important determination of the manner of death is to differentiate deaths due to natural conditions from those stemming from some nonnatural manner. Any case in which a nonnatural manner is contemplated must be referred to the appropriate medicolegal authority, medical or coroner, for review. It is important to remember that a "natural" disease may arise in a fashion that merits a nonnatural manner of death (e.g., Yersinia pestis pneumonia acquired as the result of an act of bioterrorism is homicide).

Pulmonary findings may also help resolve other medicolegal issues that may arise in a particular case. The significance of any particular finding must always be considered in the context of the issues being addressed. The significance of a particular finding can be thought of in medical/physiologic terms (i.e., what effect it has on the person) or in forensic (investigative) terms (i.e., what light it sheds on the nature, age, or cause of a disease/ 
TABLE 31.1. Death investigation process

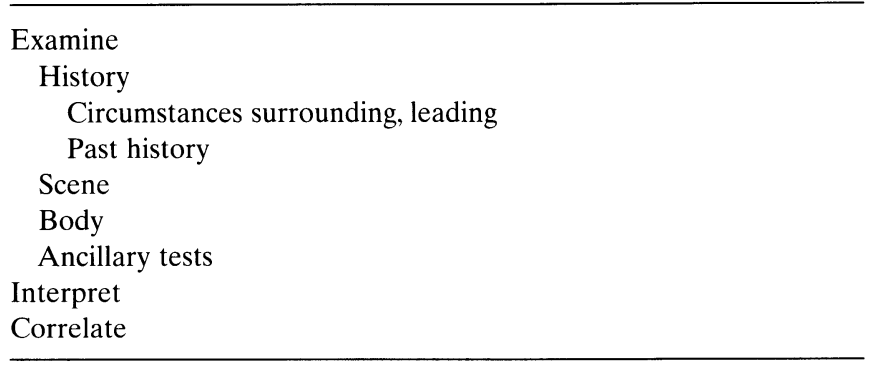

injury). The medical significance and the investigative significance can be mutually exclusive; medically important findings may be of minimal, if any, investigative help, while a medically trivial finding may be the critical datum in resolving an important investigative issue.

A variety of pulmonary lesions may be associated with or cause sudden, unexpected death. Although there are a variety of definitions of sudden death, the most commonly used refers to death occurring within 1 hour of the onset of symptoms or signs. In addition to being sudden, deaths coming under the jurisdiction of the medicolegal official, medical examiner/coroner, also are unexpected. These conditions may result from primary natural disease conditions or may arise during the course of or as sequelae to some other underlying disease or injury.

\section{Sudden Unexpected Death}

\section{Epiglottitis}

Acute epiglottitis is characterized by the abrupt onset of a clinical complex often including sore throat, dysphagia, high fever, "hot-potato" voice, drooling, and airway protective head and neck posturing. ${ }^{3}$ The disease may progress very rapidly and cause sudden obstruction of the airway. Death is typically caused by airway obstruction or sepsis. Overall, mortality is less than $5 \%$.

Haemophilus influenzae type $\mathrm{b}(\mathrm{Hib})$ is responsible for causing most cases in children and adults. In the prevaccine era (Hib vaccine was introduced circa 1985 to 1992), Hib was responsible for $75 \%$ to $90 \%$ of pediatric epiglottitis. ${ }^{3}$ Currently, $25 \%$ to $32 \%$ of cases are caused by Hib. A variety of other organisms, including Streptococcus pneumoniae, are currently responsible for a significant number of cases. ${ }^{3}$ The clinical features of epiglottitis have remained the same as before the vaccine was available. ${ }^{3}$ Introduction of the vaccine has also changed the epidemiology of epiglottitis. There has been a $95 \%$ decrease in the incidence of Hib diseases. ${ }^{4}$ In addition, the average age of patients with epiglottitis has increased. ${ }^{3}$

The gross appearance of the inflamed epiglottis differs somewhat between children and adults. In children the

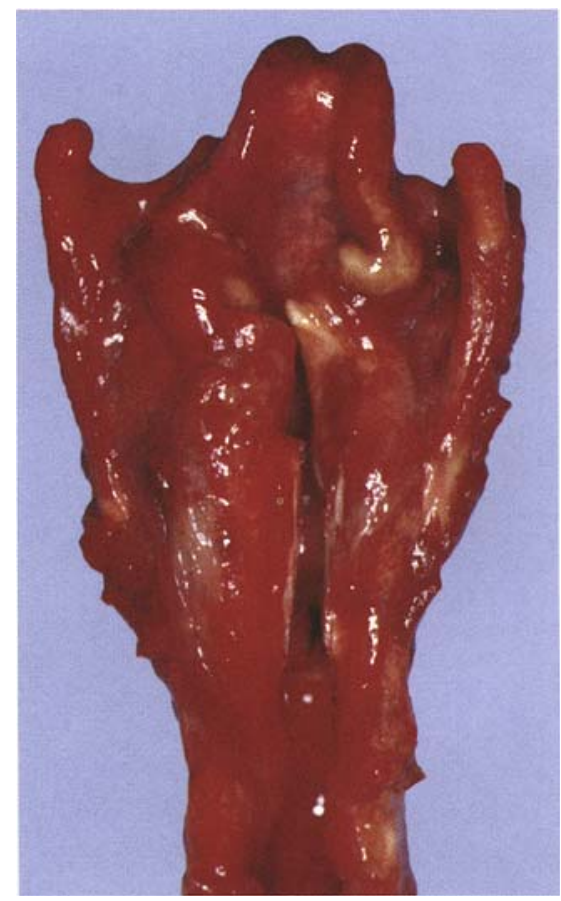

FIGURE 31.1. Acute epiglottitis. Swollen hyperemic epiglottis with focal mucosal necrosis.

epiglottis is often markedly swollen and hyperemic (Fig. 31.1), whereas in adults epiglottitis is characterized by more diffuse supraglottic swelling (Fig. 31.2). Microscopic examination demonstrates suppurative inflammation that, in some cases, may be restricted to the deep central portions of the epiglottis (Fig. 31.3).

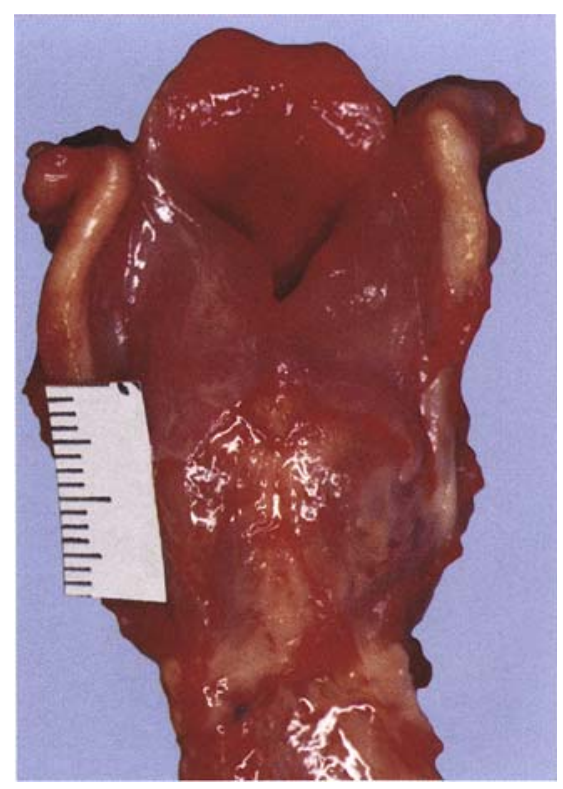

Figure 31.2. Acute epiglottitis. Diffuse supraglottic swelling. 

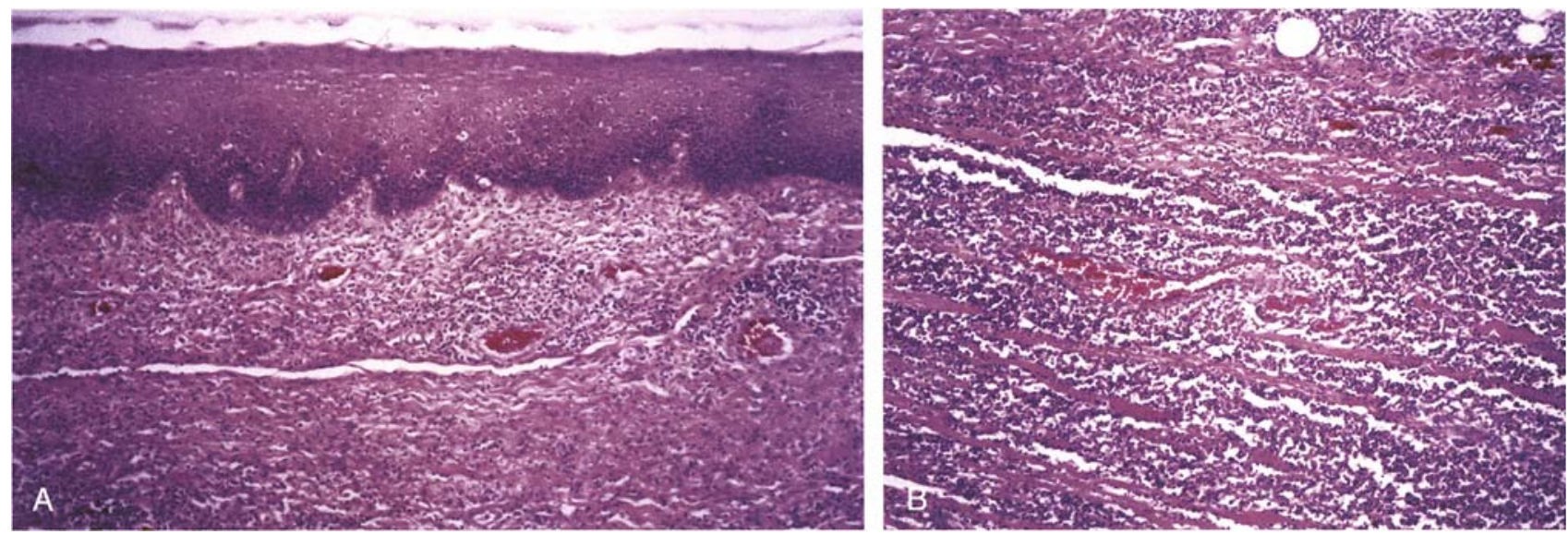

Figure 31.3. Acute epiglottitis. A. Note lack of exudate on surface. B. Acute necrotizing inflammation in the deep epiglottic tissue.

\section{Other Causes of Laryngeal Obstruction}

Tumors, cysts, and other proliferative lesions obstructing the larynx and resulting in sudden unexpected death are rare. Among these lesions are laryngeal carcinoma (Fig. 31.4), laryngeal papillomatosis, ${ }^{5}$ inflammatory polyps (Fig. 31.5), hypopharyngeal lipoma, ${ }^{6}$ laryngeal cyst ${ }^{7}$ laryngeal schwannoma, ${ }^{8}$ and prolapsed esophageal fibrolipoma. ${ }^{9}$

\section{Pneumonia}

Pneumonia is occasionally encountered as a cause of unexpected death in adults and in children. Among adults, most cases represent either fulminant pneumonia or, more commonly, pneumonia in a person who has not sought medical help until late in its course, if help was sought at all. Bacterial and viral pneumonias may present in this fashion.

Occasionally, true sudden, unexpected death may occur in an adult as a result of undiagnosed pneumonia. These individuals have minor, if any, respiratory symptoms and die suddenly while carrying out their usual daily activities. Autopsy typically demonstrates extensive pneumonia, often in a lobar distribution. In my experience, S. pneumoniae is the organism most commonly isolated from the lungs of these individuals, many of whom are chronic alcoholics. For example, a man was sitting on his usual bar

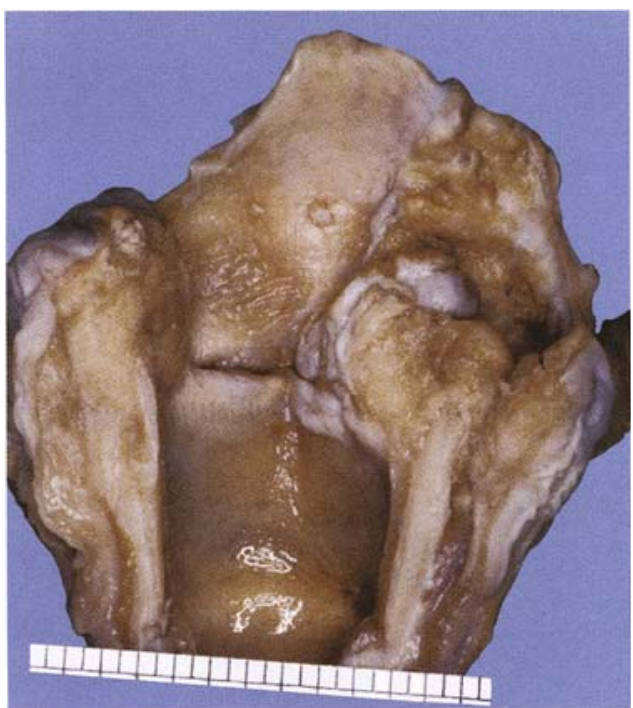

FIGURE 31.4. Laryngeal obstruction. Squamous cell carcinoma effaces the right hemilarynx.

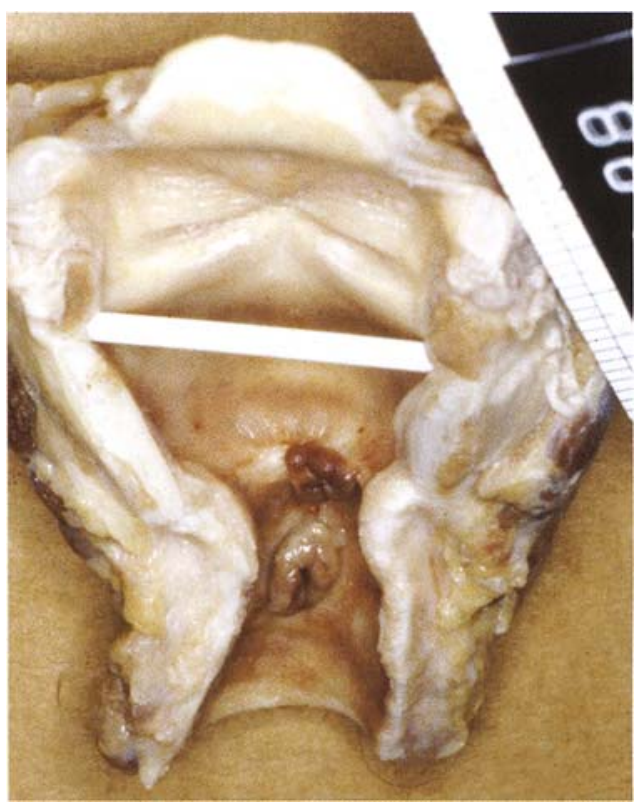

FIGURE 31.5. Laryngeal obstruction. Inflammatory polyps. 
stool in the neighborhood tavern talking and appearing normal when he suddenly collapsed to the floor, dead. The postmortem examination demonstrated acute pneumococcal pneumonia due to $S$. pneumoniae with complete consolidation of the left lung.

Similarly, it is not uncommon to find extensive pneumonia in an infant who is found dead with minimal, if any, prior signs or symptoms suggestive of significant antecedent infection. In cases in which the child displayed antemortem signs of infection, the infection was usually thought to be a "cold." ${ }^{10}$ Pneumonias resulting in sudden unexpected death in children have most commonly been the result of S. pneumoniae, Staphylococcus aureus, or Hib. ${ }^{11-13}$ The incidence of the latter has decreased markedly since the introduction of the Hib vaccine. ${ }^{14}$

\section{Bronchiolitis}

Respiratory syncytial virus (RSV) bronchiolitis rarely causes the unexpected death of an infant. In these cases the infant usually has displayed some signs of the infection. Apnea is a recognized complication of RSV infection, reported in $20 \%$ of hospitalized infants. ${ }^{15-17}$

The lungs of an infant dying of RSV infection are typically hyperinflated with areas of consolidation and hemorrhage. There is plugging of terminal and respiratory bronchioles by epithelial cell debris mixed with mucus and inflammatory cells (Fig. 31.6). There is airway epithelial hyperplasia, squamous metaplasia, and desquamation. Syncytial giant cells are in, and line, the alveolar spaces. Alveolar involvement may be extensive. There are cytoplasmic inclusions whose presence may be more readily evident in sections stained immunohistochemi-

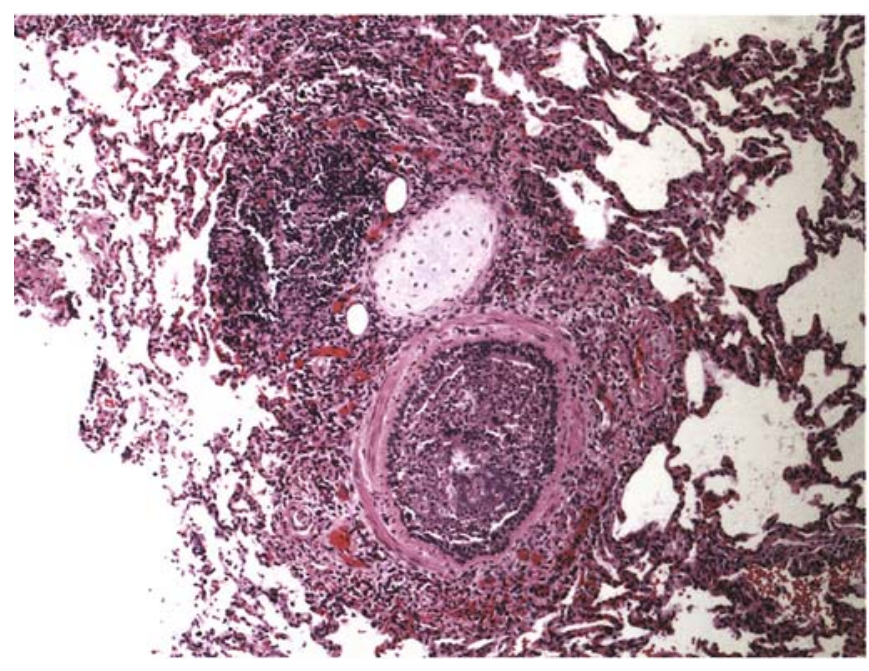

FIGURE 31.6. Respiratory syncytial virus (RSV) bronchiolitis. Bronchiolar lumen occluded by necrotic exudate. There is chronic peribronchiolitis with an adjacent lymphoid aggregate. cally with anti-RSV antibody. There may also be a peribronchial, peribronchiolar, and interstitial infiltrate of mononuclear cells and neutrophils (see Chapter 11). ${ }^{18-20}$

\section{Asthma}

Asthma is a common disease, affecting $20 \%$ of children and $5 \%$ of adults. ${ }^{21}$ Fortunately, the number of deaths attributable to asthma is relatively low. An increased risk of death has been associated with a variety of epidemiologic and clinical risk factors. ${ }^{21}$ Fatal episodes of asthma characteristically occur in patients with preexisting diagnoses of asthma (95\%), have their onset at home (56\%), and demonstrate a nocturnal predilection. ${ }^{22,23}$ Several studies have failed to demonstrate a constant seasonal characteristic in fatal asthma. ${ }^{22,24-26}$ However, other studies have reported age-specific seasonal variation and some correlation with high mold spore counts. ${ }^{27}$ Metered-dose inhalers (MDIs) are often present at the scene of an asthmatic fatality and may be found in the hand or adjacent to the body of the decedent. In some cases, evidence of the recent use of a beta-blocker may offer insight into what precipitated the terminal attack..$^{28-31}$

Overall, among patients dying of asthma there appears to be at least two subgroups that have different clinical and pathologic features. ${ }^{32,33}$ In one group, the fatal incident is of slow onset with progressive clinical deterioration that typically lasts in excess of $2 \frac{1 / 2}{2}$ hours. ${ }^{32}$ These patients, the slow-onset group, typically have a history of severe asthma, poor overall control of their disease and prolonged symptoms prior to coming for medical care during the terminal attack. Among a series of asthma deaths in Cardiff, only one third were associated with attacks lasting longer than 8 hours. ${ }^{34}$

The other group of patients succumbing to asthma, the sudden-onset group, constitutes approximately $25 \%$ of fatalities. These individuals characteristically have the sudden onset of the fatal attack and die within an hour of its onset. In the Cardiff series of asthmatic fatalities, one fourth of the deaths occurred within 30 minutes of the onset of the terminal episode. ${ }^{34}$ These persons often do not have a history of prior control problems and some have been demonstrated to have had normal airflow rates shortly prior to the onset of their lethal attack. They have a propensity to experience respiratory arrest. ${ }^{32}$ It is this group that is most often seen by medical examiners.

Readily apparent distinctive gross and microscopic autopsy findings are typically observed in persons who have died of asthma (see also Chapter 15). The lungs are usually pink and hyperinflated but have patchy areas of atelectasis. Tan viscid tenacious mucous plugs are seen in nearly $80 \%{ }^{22}$ of cases (Fig. 31.7) and may involve airways from the second generation of bronchi to the bronchioles. ${ }^{21}$ Other changes of chronic asthma are often present, including prominent bronchial gland ducts and thick 


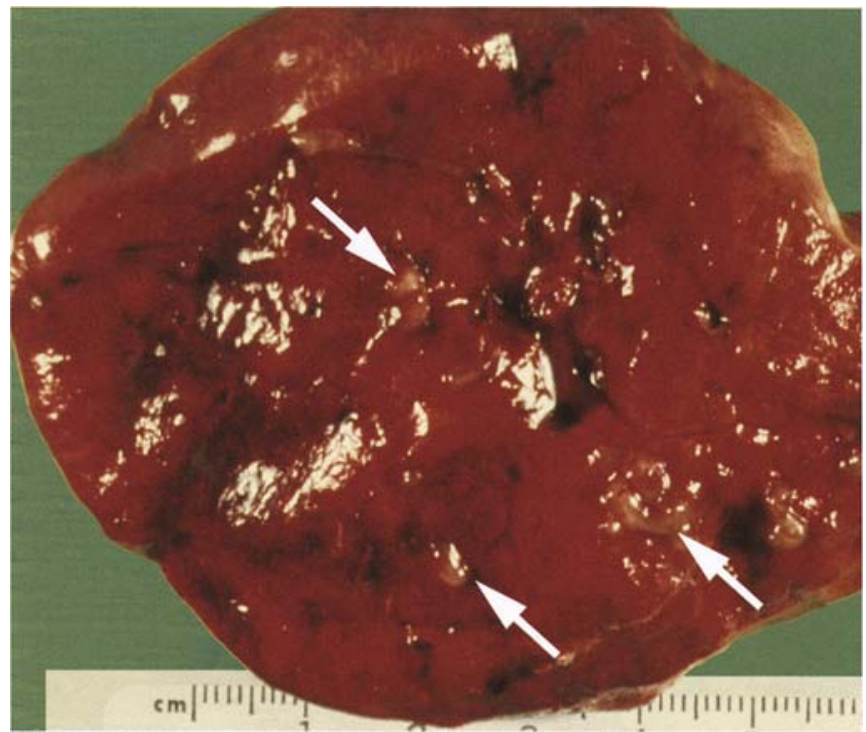

Figure 31.7. Asthma. Mucous plugs (arrows) and bronchial wall thickening are present.

bronchial walls (Fig. 31.7) ${ }^{21}$ Changes of chronic pulmonary hypertension and cor pulmonale are generally absent. ${ }^{21}$ Occasionally, significant mucous plugging is not seen. ${ }^{35-37}$ In these fatal asthmatic attacks, bronchospasm has likely played a major role in death. ${ }^{37}$

Microscopically, the lungs usually demonstrate a background of chronic asthma. Airway remodeling that may lead to irreversible airflow obstruction is more prominent in fatal cases than it is in nonfatal cases. ${ }^{38,39}$ There tends to be smooth muscle hyperplasia ${ }^{40}$ increased cartilage, increased numbers and dilation of submucosal blood vessels, ${ }^{41-43}$ and collagenous thickening of the bronchial

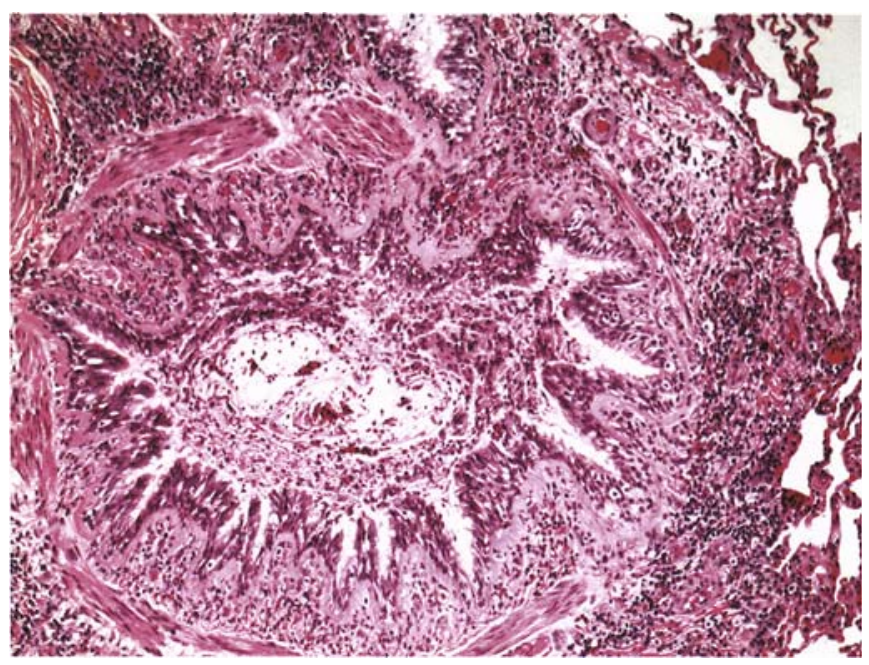

FIGURE 31.8. Asthma. Intense bronchial inflammation with occlusive luminal exudate. Note also smooth muscle hypertrophy and thickened basement membrane.

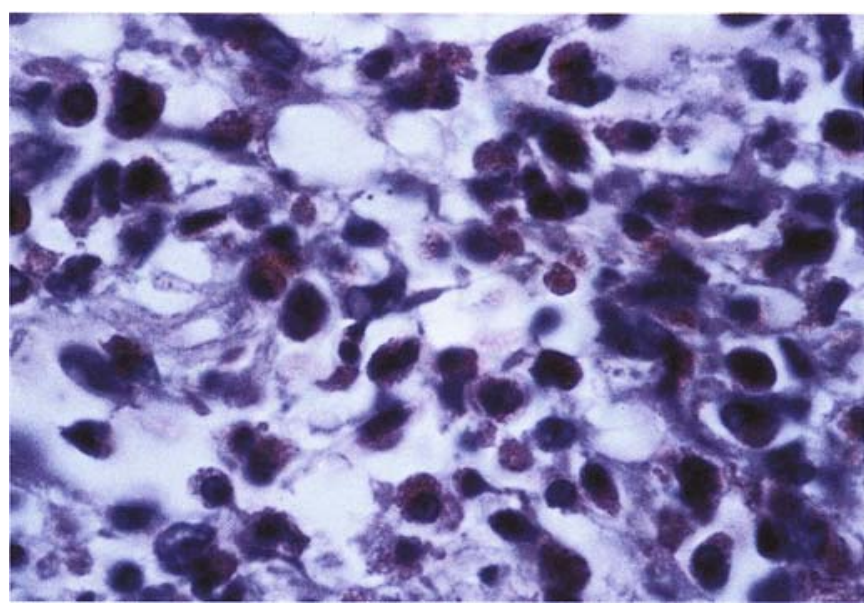

FIGURE 31.9. Asthma. Eosinophilic inflammatory infiltrate. (Giemsa stain.)

reticular basement membrane (Fig. 31.8). ${ }^{21,41,44,45}$ The latter finding has been correlated with bronchial hyperreactivity. ${ }^{46}$

Mucous plugging is usually prominent. ${ }^{21}$ The plugs consist of mucin, inflammatory cells, epithelial cells, and plasma proteins (Fig. 31.8). ${ }^{47,48}$ The increased production of mucus is likely multifactorial and includes increased secretion by goblet cells and bronchial glands along with a component of plasma proteins. Bronchial gland duct ectasia has been described in $68 \%$ of cases. ${ }^{49}$

Bronchial wall inflammation is nearly always present in asthma fatalities. Eosinophils are present in nearly $90 \%$ of cases (Fig. 31.9) ${ }^{22}$ and have expressed markers of activation. Lymphocytes and plasma cells are also usually evident and in some cases may predominate (Fig. 31.10). ${ }^{50}$

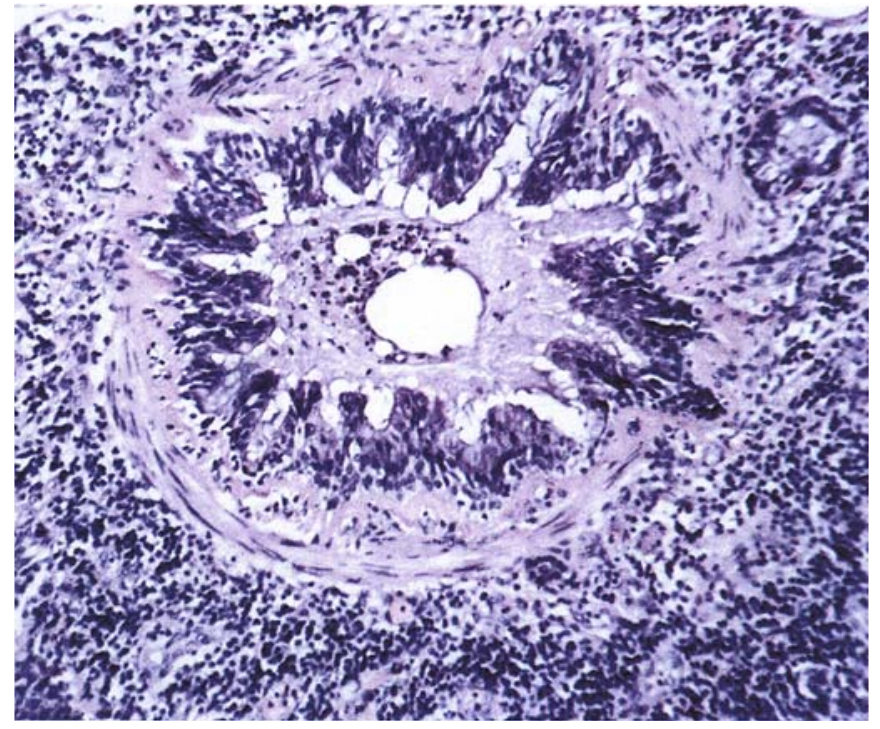

FIGURE 31.10. Asthma. Predominantly lymphoplasmacytic infiltrate. 
The lymphocytic infiltrates tend to be more prominent in the more proximal airways, and many of the lymphocytes are activated CD8 T cells. An increased number of basophils ${ }^{51}$ have also been reported in some asthma fatalities, as have neutrophils. ${ }^{52}$

The nature of the inflammatory infiltrate helps characterize the two subgroups. The number of eosinophils in the submucosa correlates with the duration of the terminal attack. Immunofluorescent studies of a series of asthma fatalities among a medical examiner office patient population demonstrated a smaller number of eosinophils and a larger number of neutrophils in the bronchial submucosa of sudden-onset episodes when compared to slow-onset cases. ${ }^{36}$ It has been postulated that the increased neutrophil population may be due to the release of neutrophil chemotactic factors after antigen or bacterial endotoxin inhalation. ${ }^{36}$

Desquamation of bronchial columnar epithelial cells is also commonly observed in the lungs of persons dying of asthma, and, as previously noted, epithelial cells are often a component of mucous plugs. ${ }^{21}$ Whether or not the epithelial desquamation seen in postmortem lung sections represents an intravitam phenomenon or a histologic artifact has been questioned. ${ }^{21}$ While some believe it is a histologic artifact, there are substantial findings that are supportive of it being an antemortem event, such as the incorporation of the epithelial cells into the mucin plug, their presence in bronchoalveolar lavage (BAL) specimens and sputum (Creola bodies) ${ }^{53}$ and the observation of regenerative changes in the residual intact epithelium. ${ }^{41}$ While it is clear that epithelial denudation is a relatively common histologic artifact, the finding of the epithelial cells in the proper context incorporated throughout the mucous plug, along with the other observations previously noted, helps in the proper interpretation of a particular case.

Asthmatic obstruction to expiratory airflow also predisposes to the development of barotrauma that can occur whether or not mechanical ventilation has been used. Pneumothorax and pneumomediastinum can occur, as can subcutaneous emphysema (see Pneumothorax and Hemothorax, below).

In assessing the role, if any, of asthma in the death of a particular person clinicopathologic correlation is essential. Individual patients can demonstrate a constellation of findings along the spectrum. While mucous plugging and inflammatory changes are more prominent in fatalities, there is no pathognomonic finding distinguishing a fatal episode of asthma from a nonfatal episode.

\section{Anaphylaxis}

Anaphylaxis is the immediate systemic hypersensitivity reaction caused by immunoglobulin $\mathrm{E}$ ( $\mathrm{IgE}$ )-triggered release of mediators from mast cells and basophils. ${ }^{54}$ It occurs from within minutes to several hours after exposure to an antigen to which there has been previous sensitization. ${ }^{54}$ A clinically identical syndrome, often called an anaphylactoid reaction, can occur from non-IgE-triggered release of these mediators. Anaphylactoid reactions can result from immune or cytotoxic complexes ${ }^{55}$ or direct mast cell degranulation.

The incidence of these reactions is not accurately known. A study in Olmstead County, Minnesota, indicated an annual incidence of $21 / 100,000$ persons and a $0.65 \%$ fatality rate. ${ }^{56}$ If this study is extrapolated to the U.S. population ( 270 million), there would be 81,000 clinical cases of anaphylaxis with 840 fatalities yearly in the United States. ${ }^{56,57}$ Fatal anaphylaxis accounts for $0.2 / 1000$ medicolegal autopsies in Sweden. ${ }^{58}$ There are some data suggesting the incidence of anaphylaxis is increasing. ${ }^{54}$

The most common triggers for fatal anaphylaxis are food (primarily peanuts and tree nuts), medications (antibiotics, primarily parenteral; allergy shots) ${ }^{56,59-61}$ and insect stings. Fatal anaphylaxis precipitated by allergen immunotherapy occurs at an estimated rate of 1 case per 2 million injections. No triggering agent is identifiable in approximately $25 \%$ to $33 \%$ of anaphylaxis cases. ${ }^{55}$

Triggers for IgE-independent reactions (anaphylactoid reactions) include, among others, radiocontrast media, angiotensin-converting enzyme (ACE) inhibitor administered during renal dialysis, opioids, immune aggregates, exercise, and temperature (hot, cold) ${ }^{62,63}$

Regardless of whether the reaction is $\operatorname{IgE}$ mediated or not, the clinical syndrome of "anaphylaxis" results from the effects of the release of a variety of mast cell/basophil mediators-primarily histamine. Serum histamine concentrations correlate with the severity of cardiopulmonary and gastrointestinal manifestations but not with the formation of urticaria. ${ }^{64,65}$ Other mediators that are released include serotonin, $\beta$-tryptase, chymase, heparin, cytokines, and leukotrienes. ${ }^{57}$

The clinical severity of any particular attack is not predictable. Although the severity of the reaction is usually dose dependent, exposures to small amounts of antigen can precipitate severe attacks in highly sensitized individuals.

Signs and symptoms of anaphylaxis may involve multiple organ systems. The respiratory, cardiovascular, musculocutaneous, and gastrointestinal systems are most commonly involved. The primary shock organs in humans are the lungs and heart, with death being the result of respiratory obstruction or cardiovascular collapse. $^{66}$

The clinically apparent onset of an anaphylactic attack commonly occurs within minutes of exposure to the inciting agent; however, the onset may be delayed for several hours. The attack is usually monophasic and 


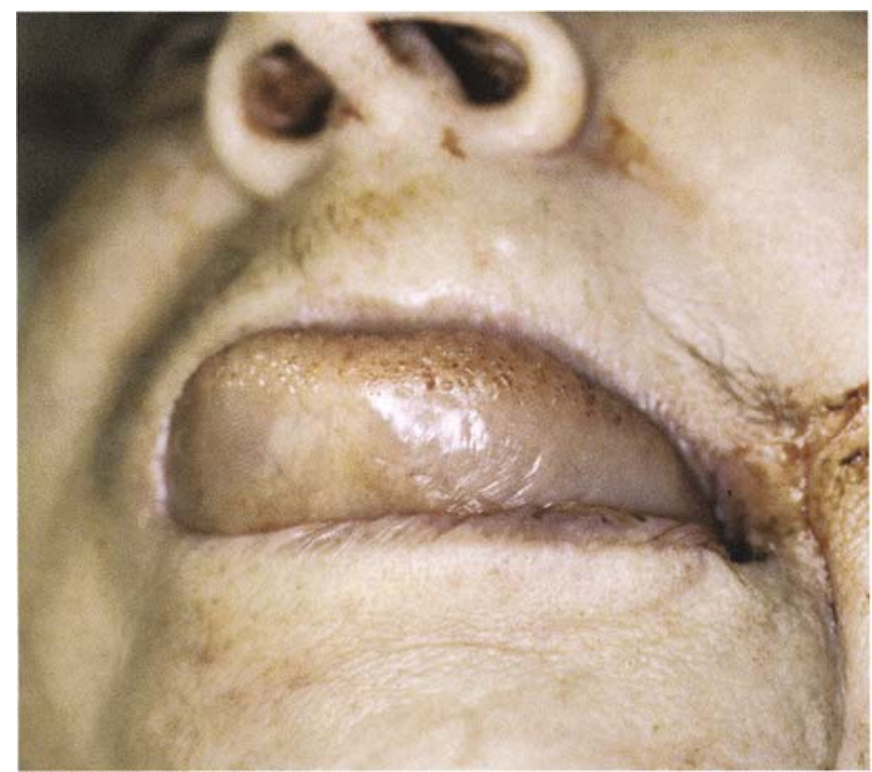

FIGURE 31.11. Anaphylaxis. Oral angioedema with protrusion of swollen tongue.

resolves within 2 hours after it is treated. In occasional cases the respiratory or cardiovascular manifestations may persist for more than 10 hours ("protracted" anaphylaxis) ${ }^{54}$ Occasionally, the attack may be biphasic (5\% to $20 \%$ of cases $)^{67-69}$ with a recrudescence of signs/symptoms, often occurring 8 to 12 hours later, in the absence of reexposure to the triggering agent.

Respiratory dysfunction is evident in $69 \%$ of adults and $93 \%$ of children. Both the upper and lower portions of the respiratory tract can be affected by anaphylaxis, resulting in nasal congestion, sneezing, hoarseness, stridor, cough, obstruction, tight throat, laryngeal edema, dyspnea, bronchospasm, tachypnea, wheezing, cyanosis, and respiratory arrest. ${ }^{54}$ Shortness of breath is almost always present in attacks triggered by food. Cutaneous involvement is evident in nearly all cases involving adults and
$93 \%$ of those in children. Dermatologic findings include urticaria, angioedema, pruritus, flushing, conjunctivitis, and chemosis. ${ }^{54}$

Cardiovascular abnormalities are clinically apparent in $41 \%$ and $26 \%$ of adults and children, respectively. ${ }^{54} \mathrm{Car}-$ diovascular manifestations of anaphylaxis include tachycardia, presyncope/syncope, hypotension, shock, chest pain, bradycardia, orthostasis, and cardiac arrest. ${ }^{54}$ Shock occurring in the absence of shortness of breath is most commonly associated with attacks triggered by venom and iatrogenic agents. Gastrointestinal signs and symptoms are seen in $24 \%$ of adults and $13 \%$ of children. Anaphylaxis may manifest in the gastrointestinal tract as intraoral angioedema (Fig. 31.11), emesis, nausea, cramps, dysphagia, and diarrhea. ${ }^{54}$

The diagnosis of an anaphylactic or anaphylactoid reaction rests on a constellation of findings. Factors suggesting the diagnosis include a past history of attacks, immediate prior challenge to a known triggering material, characteristic clinical signs/symptoms, demonstration of specific $\operatorname{IgE}$ antibodies (anaphylaxis) and elevated concentration of blood tryptase.

The diagnosis of death due to anaphylaxis, especially when the event is unwitnessed, may be problematic. ${ }^{70}$ There are no pathognomonic findings in anaphylaxis, and autopsy findings, when present, are often nonspecific. Findings may be affected by the type of allergin, route of administration, and duration of the anaphylactic episode. ${ }^{58}$ In a series of 56 anaphylaxis fatalities, postmortem findings included pharyngeal/laryngeal edema (in 23 patients) (Fig. 31.12), mucous plugging/emphysema (in 15), and petechiae (in 10).$^{71}$ Occasionally, a sting site can be identified or the ingested antigen (e.g., peanut) may be seen in the gastrointestinal tract. ${ }^{72}$ Although nonspecific and inconstant, the presence of mucosal edema (Figs. 31.11 and 31.12) and mast cell infiltration with degranulation support the diagnosis of anaphylaxis. ${ }^{72}$ A paucity of postmortem findings is especially likely in cases characterized by the rapid onset of shock. ${ }^{71}$
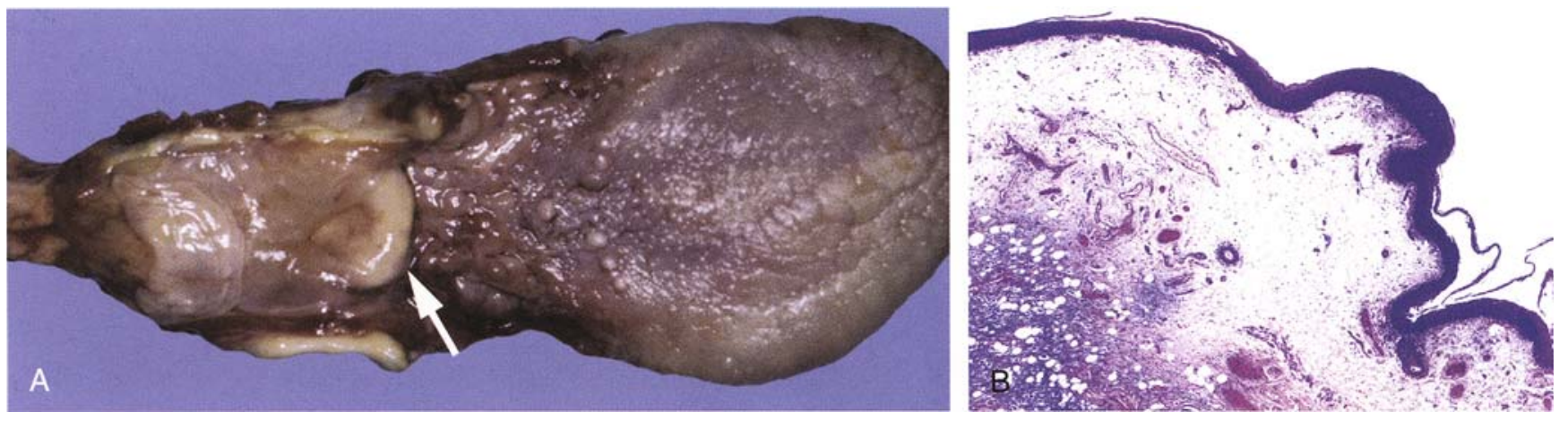

Figure 31.12. Anaphylaxis. A. Pharyngeal and laryngeal edema. Note markedly swollen epiglottis (arrow). B. Submucosal edema and lymphangiectasia of epiglottis. 
Laboratory studies may be very helpful in the proper assessment of anaphylaxis, including the use of postmortem chemical studies in evaluating suspected cases of fatal anaphylaxis. ${ }^{71}$ Measurement of histamine in postmortem blood has not been helpful in establishing the diagnosis of anaphylaxis due to the short half-life of histamine and its postmortem degradation. ${ }^{72,73}$ In addition, blood histamine concentration has been increased by nonimmunologic means including postmortem release. ${ }^{73}$ Tryptase is a neutral protease and is the only protein that is concentrated selectively in the secretory granules of human mast cells and is released when mast cells degranulate. ${ }^{70,72}$ Elevated serum tryptase may not be detected during the first 15 to 30 minutes after the onset of an anaphylactic event. Peak plasma concentration of tryptase occurs in 1 to 2 hours and declines with a half-life of 2 hours. ${ }^{73}$ Plasma concentration of tryptase, especially $\beta$-tryptase (the form stored in cytoplasmic granules), correlates with the clinical severity of anaphylaxis. The demonstration of an elevated plasma tryptase concentration in a blood sample obtained during life is highly specific for anaphylaxis. ${ }^{70}$

The interpretation of tryptase concentrations in postmortem blood samples is not so straightforward. ${ }^{72}$ Elevated concentrations of blood tryptase have been demonstrated in nearly $90 \%$ of anaphylaxis fatalities. However, elevated blood tryptase concentrations have also been found in postmortem samples obtained from persons whose deaths were not attributed to anaphylaxis, ${ }^{70}$ including cases with chest trauma and arteriosclerotic cardiovascular disease..$^{70,72}$. Randall et al. ${ }^{70}$ reported elevated (greater than $1 \mathrm{ng} / \mathrm{ml}$ ) blood tryptase concentrations in $63 \%$ of nonanaphylaxis deaths (31 of 49 ) with two of the cases (4\%) measuring 5.1 to $10 \mathrm{ng} / \mathrm{mL}$ and five cases $(10 \%)$ being $>10 \mathrm{ng} / \mathrm{mL}$ ). A study of 193 postmortem cases demonstrated a sensitivity of $86 \%$ and specificity of $88 \%$ when a $10-\mathrm{ng} / \mathrm{mL}$ concentration cutoff was used. ${ }^{74}$ Normal postmortem concentrations may be seen in anaphylaxis deaths when death is very rapid and when death ensues after a prolonged survival interval.

When faced with a potential anaphylaxis fatality, the pathologist should strive to obtain any antemortem blood samples that may be available. In addition, a postmortem sample of blood should also be obtained for tryptase analysis. The blood sample should be obtained as soon after death as possible and stored frozen at $-20^{\circ} \mathrm{C}^{75}$ Some authors have reported artifactual elevation of postmortem blood tryptase in samples obtained 14 or more hours after death. ${ }^{76}$ Other studies have found no correlation with the postmortem interval. ${ }^{74}$ Femoral vein blood also appears to be the postmortem specimen of choice since it has been found that heart blood tryptase was elevated more often than femoral vein blood tryptase. $^{58}$
In addition to tryptase analysis, blood (antemortem or postmortem) may be submitted for a radioallergosorbent test (RAST) to identify specific IgE antibodies. The presence of an elevated RAST for a particular substance confirms prior sensitization to that allergen. ${ }^{77}$ A RAST directed toward a panel of antigens may be helpful in recognizing the responsible antigen in cases where there may also be moderate elevation of specific IgE to related but distinct antigen classes. ${ }^{72}$

The use of total IgE concentration in the blood has also be postulated to have a role in the postmortem evaluation of anaphylaxis. ${ }^{77,78}$ However, its utility in confirming anaphylaxis is questionable. Total IgE concentrations among atopic individuals are variable. In addition, elevated $\operatorname{IgE}$ has been reported in the settings of trauma, sepsis, and other nonatopic conditions. ${ }^{72}$

As with many other entities causing sudden unexpected death, there is no "magic bullet" establishing the diagnosis of fatal anaphylaxis. Arriving at the correct diagnosis requires a high index of suspicion coupled with careful clinicopathologic correlation. ${ }^{71}$

\section{Primary Pulmonary Hypertension}

Primary (idiopathic) pulmonary arterial hypertension (PPH) is a condition characterized by sustained elevations of pulmonary arterial pressure (exceeding $25 \mathrm{~mm} \mathrm{Hg}$ at rest or exceeding $30 \mathrm{~mm} \mathrm{Hg}$ with exercise) without a demonstrable cause. ${ }^{79,80}$ The pathogenesis of increased pulmonary vascular resistance in patients with $\mathrm{PPH}$ involves vasoconstriction, vascular wall remodeling, and in situ thrombosis. A variety of conditions with similar clinical and pathologic features to $\mathrm{PPH}$ have been identified. ${ }^{81}$

Primary pulmonary hypertension is typically a disease of young women ${ }^{82,83}$ with an incidence of 1 to 2 per million persons in the general population. ${ }^{84,85}$ Familial cases accounted for $6 \%$ of cases in a National Institutes of Health (NIH) registry. ${ }^{79}$ Sudden death is a relatively common manifestation of this entity, occurring in $7 \%$ of cases, ${ }^{81}$ and, uncommonly, may be its initial presentation. ${ }^{82,86}$ Sudden death may be precipitated by stress that would ordinarily be considered inconsequential. Rarely, PPH may manifest itself as sudden death due to pulmonary artery dissection. ${ }^{87}$ For further discussion of PPH, see Chapter 28.

\section{Embolism}

A variety of solid materials may flow through the systemic venous system to eventually lodge in the pulmonary vasculature. The effects these materials have on the lungs depend on the type and amount of the material, chronicity of exposure, and comorbid conditions. The 
various embolic substances are typically associated with characteristic scenarios.

\section{Pulmonary Arterial Thromboembolism}

The incidence of pulmonary arterial thromboembolism (PTE) in the United States has been estimated at 600,000 per year. ${ }^{88}$ The true incidence of PTE is difficult to ascertain due to diagnostic difficulties in establish the diagnosis clinically and due to population bias in the studies. It is estimated that only $30 \%$ of PTE are diagnosed antemortem. ${ }^{89}$ Untreated, mortality has been reported as $20 \%$ to $30 \%$. With treatment, mortality decreases to $2.5 \% .{ }^{90-93}$ The incidence reported in autopsy studies also varies, reflecting different patient populations (notably, hospital versus medical examiner) and the extent of the examination used to detect thromboemboli. Pulmonary thromboembolism has been reported in $17 \%$ of hospital autopsy series and has been cited as the primary cause of death in $0.8 \%$ of medical examiner autopsies. ${ }^{94,95}$

The formation and propagation of venous thrombi are favored by abnormal flow or stasis, an abnormal blood vessel wall, and hypercoagulability-Virchow's triad.

A variety of risk factors have been recognized that predispose to the development of deep venous thrombosis (DVT) ${ }^{96,97}$ Among these factors are prolonged immobilization, recent surgery (3 months), pelvic/lower extremity trauma, venous obstruction by external compression, cancer (especially adenocarcinoma), previous DVT, and thrombophilia. ${ }^{98-102}$ Overall, $80 \%$ to $96 \%$ of patients with PTE have at least one major risk factor. ${ }^{103,104}$ In a series of medical examiner cases in which death was attributed to PTE, $86 \%$ of the cases were associated with a recognized cause or risk factor, most commonly obesity $(24 \%)$, immobilization $(23 \%)$, or trauma $(14 \%)$. No risk factor was recognized in $14 \%$ of these deaths. ${ }^{105}$

Occasional cases of PTE have been ascribed to some inherited form of thrombophilia. In a series of 34 PTE deaths selected for molecular screening $(80 \%$ selected due to age $<45$ years), three were found to be heterozygous for the prothrombin G2010A mutation and one for factor $\mathrm{V}$ Leiden mutation. An additional eight cases were heterozygous and one was homozygous for mutation of methylenetetrahydrofolate reductase (MTHFR) ${ }^{95}$

The root of most inherited thrombophilias is impaired thrombin neutralization or failure to control thrombin generation. ${ }^{106}$ The former is characteristic of antithrombin deficiency and the latter is associated with deficiencies of protein $\mathrm{S}$ or protein $\mathrm{C}$ or the presence of factor $\mathrm{V}$ Leiden or prothrombin G20210A mutations. ${ }^{107}$ More than half of inherited thrombophilias are due to factor $\mathrm{V}$ Leiden mutations and, to a lesser extent, the prothrombin G20210A mutation. The heterozygote state for factor $\mathrm{V}$ Leiden mutation has a sevenfold increased risk for the development of DVT, whereas the risk in the homozygote is increased 100 times. $^{108}$ The heterozygous state for the prothrombin G20210A mutation carries an increased risk for DVT/PTE of about 4. Mutation of MTHFR is very common (heterozygous in $40 \%$ to $50 \%$ of Caucasians) but is of uncertain clinical significance. It does not appear to confer independent risk for the development of DVT/PTE. Any increased risk associated with this mutation appears to be dependent on serum folate and $\mathrm{B}_{12}$ concentrations. ${ }^{95}$

Inherited thrombophilia should be considered in recurrent episodes of DVT/PTE, a family history of DVT/PTE, age less than 45 years, "soft" or inapparent risk factors, or a history of multiple second- or third-trimester stillbirths/ abortions. In these cases, an antemortem or postmortem molecular thrombin profile should be considered. ${ }^{107}$

Deep venous thrombosis predominately arises in the veins of the lower extremities, most often starting in the calf veins. ${ }^{109}$ In $10 \%$ to $20 \%$ of cases the thrombus extends proximally. Fatal PTE occurs in $1 \%$ to $5 \% .{ }^{97}$ A significant number of these deaths are sudden. Nearly $75 \%$ of PTE deaths occur within the first 24 hours after the embolism, with one third of them occurring in the initial hour. ${ }^{110}$ The incidence of fatal PTE can be decreased through the use of preventative therapy.

An embolus travels from its site of origin via the systemic venous circulation, through the heart, to the pulmonary arterial system, where it lodges. Increased pulmonary vascular resistance with, in some cases, right ventricular dysfunction, is due to mechanical vascular obstruction plus the effect of the vasoactive mediator release. The clinical effect of the embolism depends on the size of the thrombus and the degree of pulmonary reserve. ${ }^{111-114}$

Fatal PTE in otherwise healthy persons typically involves obstruction of the extrapulmonary portions of the pulmonary artery or proximal intrapulmonic segments (Fig. 31.13; also see Fig. 28.22 in Chapter 28). More limited pulmonary arterial obstruction may lead to death in seriously ill patients or in those with limited cardiopulmonary reserve.

A number of medicolegal issues may need to be addressed when evaluating a death that may have been due to PTE. Although many cases of sudden death occur in ambulatory patients without previous disease or injury, a substantial number will arise in or out of the hospital during convalescence from some other disease or injury. In these cases, it is essential that the underlying condition complicated by the development of DVT/PTE be recognized so that the underlying cause of death may be accurately identified and the death certified appropriately. This may have profound effects on the decedent's estate, insurance, and civil or criminal legal proceedings. For example, a person lying in a hospital bed for 2 weeks during recovery from a pelvic fracture sustained in a motor vehicle crash develops lethal DVT/PTE. In this 


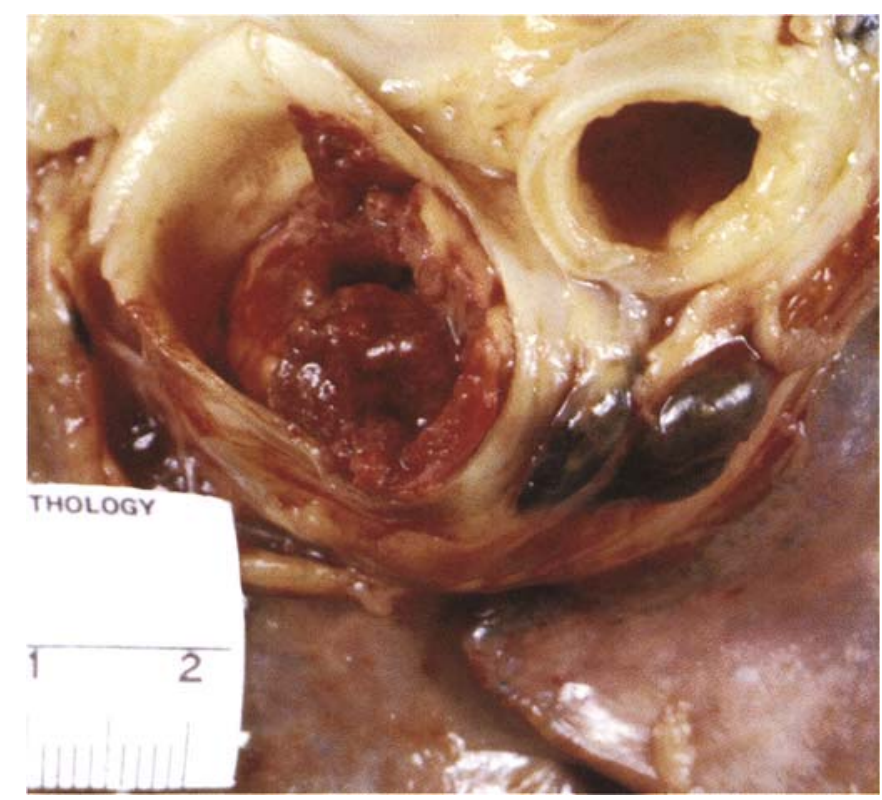

FIGURE 31.13. Acute pulmonary thromboembolus occluding hilar pulmonary artery. Note vague linear striations (lines of Zahn).

case, death should be certified as PTE due to DVT due to pelvic blunt trauma. The manner of death is accident.

When evaluating the pulmonary vasculature for the presence of thrombi, the pathologist must ascertain if a particular mass of blood is a clot, thrombus (in situ) or thromboembolus (see Table 28.6 in Chapter 28).

On gross examination, a large pulmonary artery thromboembolus may be twisted upon itself, and its configuration may help identify its site of origin, such as the deep veins of the lower extremities (see Fig. 28.22B in Chapter 28 ). The thrombus may show the configuration of the sinuses of the venous valves where it formed. There may be a corrugated or ridged appearance of parts of the thrombus where the lines of Zahn come to the surface. A thrombus is typically firm, fills the lumen, and may distend the vessel (Fig. 31.13). In contrast, most clots do not fill the vessel; they conform to the pattern of the vessel, often have distinct cellular and coagulated liquid components ("cranberry jelly" and "chicken fat"), and tend to be soft and easily compressed. ${ }^{1}$

Differentiation of thrombus from thromboembolus can be difficult. An in situ thrombus is not coiled on itself, whereas a thromboembolus may be. In addition, a large pulmonary artery thrombosis typically is associated with underlying destructive lung disease (see Fig. 28.32 in Chapter 28). In some cases it may be possible to trace the thrombus to an area of parenchymal destruction and frequently demonstrate the presence of endarteritis obliterans. ${ }^{115}$

Determining the age of a thromboembolus is a common medicolegal problem. In essence, there are two issues in this determination: the age of the thrombus and the age of the embolization (see Table 28.7 in Chapter 28). In general, the dating of a thrombus depends on an assessment of the degree of preservation of the original constituents of the thrombus and the degree of organization of the thrombus by ingrowth of mesenchymal elements from the tissue underlying the thrombus at its site of origin. Mononuclear blood cells in the thrombus may also play a role in organization. These cells multiply by mitosis and differentiate into macrophages, endothelial cells, fibroblasts, and smooth muscle cells. ${ }^{116}$ The consequences of this organization may vary depending on the site at which the thrombus forms.

Similar considerations apply to dating of thromboemboli but, of course, one cannot always be certain as to how long a thrombus was present in its site of origin prior to embolizing. It is reasonable to expect that a thrombus remaining at the site of origin will acquire ingrowth of mesenchymal cells, become mechanically attached to the site, and be less capable of embolizing as time passes. Experience with cases of rapidly fatal pulmonary thromboemboli suggests that most such thrombi (or at least a segment of a thrombus) had formed within at most a few days prior to their embolization. ${ }^{1}$

Thromboemboli resolve by fibrinolysis, retraction, and organization. The sequential process of organization by mesenchymal cells from the pulmonary artery at the site of lodgment lends itself to age assessment. Dating of an embolus is most reliably accomplished by demonstrating and assessing attachment of the embolus to the pulmonary arterial wall. To best accomplish this, it is important for the pathologist to identify and sample points of adherence between the embolus and the vessel wall in order to

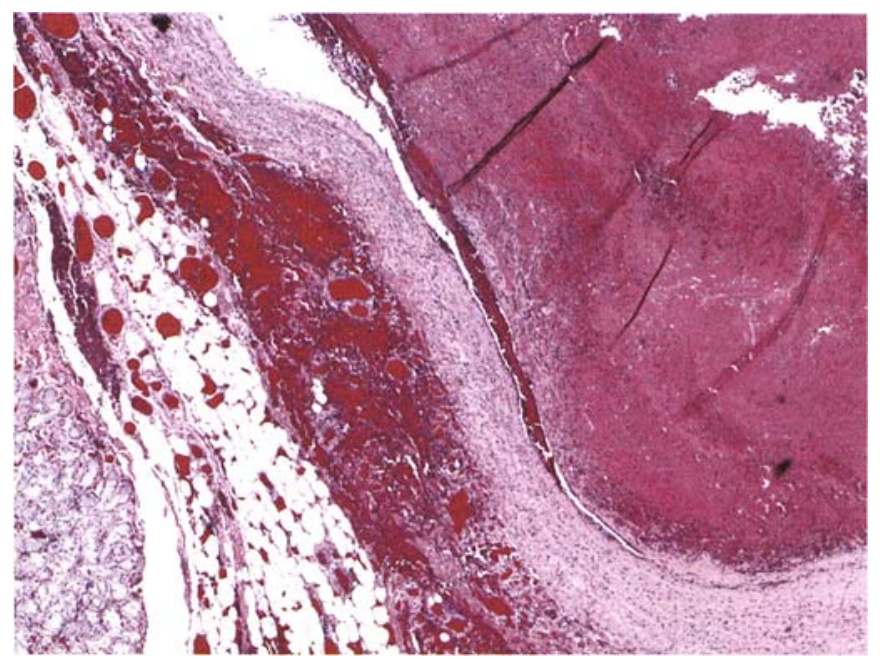

FIGURE 31.14. Organizing pulmonary thromboembolus with fibrous attachment to intimal surface. Note also adventitial congestion and hemorrhage. 

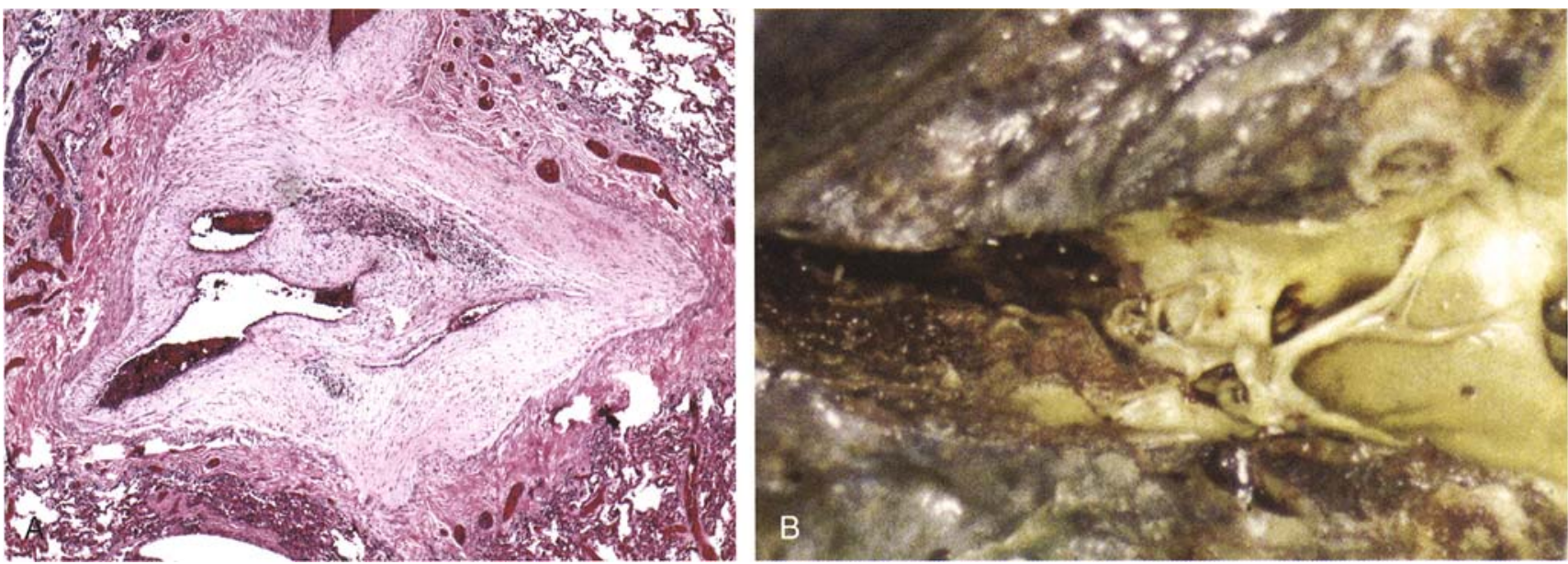

Figure 31.15. A. Remote recanalized pulmonary thromboembolus. B. Organizing thromboembolus (left) proximal to an intravascular fibrous cord.

ensure that only areas of more recent intrapulmonary propagation of the thrombus are not sampled thus resulting in underestimation of the true age of the entire thromboembolus (see Table 28.7 in Chapter 28).

In general, the vasa vasorum at the point of thromboembolus lodgment is dilated, and fibrin is deposited on the surface of the thromboembolus within a few hours of impaction. By 24 hours, the adjacent pulmonary artery media is edematous and infiltrated by neutrophils, and there are endothelial cells on the surface of the embolus (see Figs. 28.23 and 28.24 in Chapter 28). Fibrous tissue at the point of attachment and endothelial-lined channels are seen by 7 days (Fig. 31.14). The thromboembolus, especially one in a smaller conducting artery, shows extensive fibrosis in 2 to 3 weeks. Final resolution may result in a fibrous plug within the lumen that may or may not recanalize (Fig. 31.15A), incorporation into the artery wall resulting in a fibrous intimal plaque, or formation of a fibrous band or web (Fig. 31.15B; see also Fig. 28.11 in Chapter 28). Complete resolution can occur in 2 to 3 weeks, with $60 \%$ completely resolved within 4 months after a major embolic event. ${ }^{110}$ Pulmonary artery thromboemboli are less commonly seen in muscular arteries, but the sequence of organization appears similar to that in elastic arteries. ${ }^{117}$ The shear force of flowing blood maintains the lining cells of the cardiovascular system in a flattened state. When this shear force is reduced or eliminated, the cells round up and proliferate. With thrombosis, the shear effect of the flowing blood is lost, and the lining cells are free to proliferate into the overlying thrombus. This organization begins as a loose network of proliferating mesenchymal cells, becomes vascular granulation tissue, and later becomes dense scar that may be recanalized (see Fig. 28.24 in Chapter 28). If the surface of the thrombus becomes endothelialized, it will mature into dense connective tissue. If the surface does not endo- thelialize, the thrombus will continue to accumulate new thrombotic material on its surface. Thus, in cardiac or aortic aneurysms, it is common to see thrombus that is months to years old in the deepest parts adjacent to the wall, and only hours to days of age on the surface exposed to the flowing blood. A similar phenomenon may be seen in elastic pulmonary arteries, where thromboemboli may be associated with damage to the arterial wall and aneurysm formation. The mechanical impact of the thromboembolus may acutely stretch and damage the vessel wall, which then dilates to form an aneurysm. The morphologic appearance is that of advanced organization adjacent to the vessel wall and superficial fresh thrombus on the luminal surface. ${ }^{1}$

Most pulmonary artery thromboemboli, even those that are occlusive, do not produce infarction of the pulmonary parenchyma. When a thrombus occludes a pulmonary artery branch, the bronchial circulation is usually able to supply adequate blood flow to the involved parenchyma through collateral connections. If a person having left-sided congestive heart failure sustains a pulmonary artery thromboembolus, flow through the bronchial collaterals may be inhibited and the lung tissue may infarct (see Chapter 28).

The pathologist should also attempt to ascertain if there is any evidence indicating the presence of previous pulmonary emboli. Most cases of fatal PTE demonstrate evidence of previous thromboemboli, recent or remote. In a series of deaths caused by massive PTE, 13 of the 14 cases had evidence indicative of prior embolic episodes. ${ }^{118}$

\section{Fat Embolism}

The initial observation of pulmonary fat embolism (PFE) is credited to Zenker ${ }^{119}$ in 1862 . Recognition of the clinical fat embolism syndrome (FES) soon followed. ${ }^{120}$ 
PFE is most commonly associated with blunt force injury (BFI). ${ }^{121}$ In a recent histologic study, PFE was seen in $68 \%$ of BFI fatalities and in $15 \%$ of non-BFI deaths. The extent of fat embolism (FE) in BFI deaths exceeded that in non-BFI cases. ${ }^{122}$ The non-BFI deaths had undergone cardiopulmonary resuscitation (CPR) or had marked liver steatosis. ${ }^{122}$ Although pulmonary fat embolism is commonly seen in cases of significant blunt trauma, the FES is relatively uncommon. ${ }^{123,124}$ The FES is most likely to occur after fractures of the pelvis and lower extremity long bones. ${ }^{124}$ It has been also been associated with a wide variety of other conditions, many of them nontraumatic. $^{125}$

Signs and symptoms of the FES typically appear after a 6 - to 12 -hour latency period, with $60 \%$ occurring within 24 hours and $85 \%$ having their onset within 48 hours. ${ }^{126-130}$ Although there are a variety of clinical features, the most important diagnostic signs and symptoms of the FES involve the lungs, brain, and skin. Dyspnea and tachypnea develop in $75 \%$ of FES patients with $10 \%$ of them developing respiratory failure. ${ }^{131}$ An early, almost invariable, finding is a decreased arterial $\mathrm{pO}_{2}{ }^{132,133} \mathrm{~A}$ "snowstorm" radiographic pattern is seen in $30 \%$ to $60 \%$ of cases. ${ }^{134}$ Anoxia due to vascular obstruction leads to cerebral dysfunction in $86 \%$ of FES patients. ${ }^{134}$ The skin is the third major tissue characteristically affected in FES. A patchy, fleeting petechial rash is seen, often in nondependent areas, in $20 \%$ to $50 \%$ of cases. ${ }^{135,136}$ The rash typically develops after 48 to 72 hours. ${ }^{125}$ The FES usually resolves in 3 to 7 days unless complications arise. The prognosis is good if the initial respiratory insult is survived. ${ }^{125}$ Mortality during the 1960 s and 1970 s was estimated at $10 \%$ to $20 \%$ but has subsequently decreased primarily due to improvements in therapy.

The source of the FE and the pathogenesis of the FES are unclear. Two theories are commonly cited: mechanic and biochemical. However, these theories are not necessarily mutually exclusive. ${ }^{125}$ In the mechanical theory, ${ }^{124}$ large fat particles occlude the small pulmonary vessels while small fat globules pass through the lung capillaries and enter the systemic circulation. Some authors have correlated the amount of fat with respiratory dysfunction, while others dispute any correlation. The source of the fat has been postulated as the bone marrow and the soft tissues adjacent to injured bones. Some studies report other bone marrow elements along with the fat in the pulmonary vasculature, ${ }^{124,137-139}$ whereas others report minimal or no other bone marrow elements, ${ }^{122,140}$ an observation proposed as support for soft tissue being the source of the fat. ${ }^{122}$ On the other hand, the composition of the fat in the lung has been reported as being the same as that in bone marrow. ${ }^{141,142}$ Also, the observation that the FES is about 100 times more common in adults than it is in children has been attributed to differences in the fat content and composition of the bone marrow between adults and children. ${ }^{143-145}$

The other major theory proposed to explain the development of FES is the biochemical theory. ${ }^{124}$ According to this theory, destabilization of a plasma chylomicron emulsion leads to coalescence of fat stores. ${ }^{124,146}$ The progression from a case of fat embolism to one of FES may be explained by a biochemical process having a role in the development of the FES. Fatty acids are toxic to endothelial cells and pneumocytes. ${ }^{124}$ In addition, interleukin- 6 (IL-6) may be responsible for the fever that is commonly seen in FES. ${ }^{124}$ The IL- 6 may be due to increased production of tumor necrosis factor- $\alpha$ (TNF- $\alpha$ ) and IL- $1 \beta$, possibly related to injury to soft tissue. ${ }^{124}$

Pulmonary fat embolism can be seen in hematoxylin and eosin (H\&E)-stained sections of lung tissue (Fig. 31.16). Fat can also be visualized using special stains. A number of special staining techniques, each with advantages and disadvantages, have been described. Perhaps the classic special staining technique for fat embolism is the use of oil red $\mathrm{O}$ on frozen sections of lung tissue (Fig. 31.17). This technique is plagued with poor tissue detail and displacement of the fat due to lack of lipid immobilization during processing. ${ }^{147}$ Fixation of lung tissue with osmic acid has also been proposed as a method for demonstrating fat in paraffin-embedded tissue. This method is expensive, has poor tissue penetration, uses toxic reagents, and fails to reveal saturated fats. ${ }^{147}$ More recently, an inexpensive, although slow (due to long waiting steps), staining method using chromic/linoleic acid has been described. ${ }^{147}$

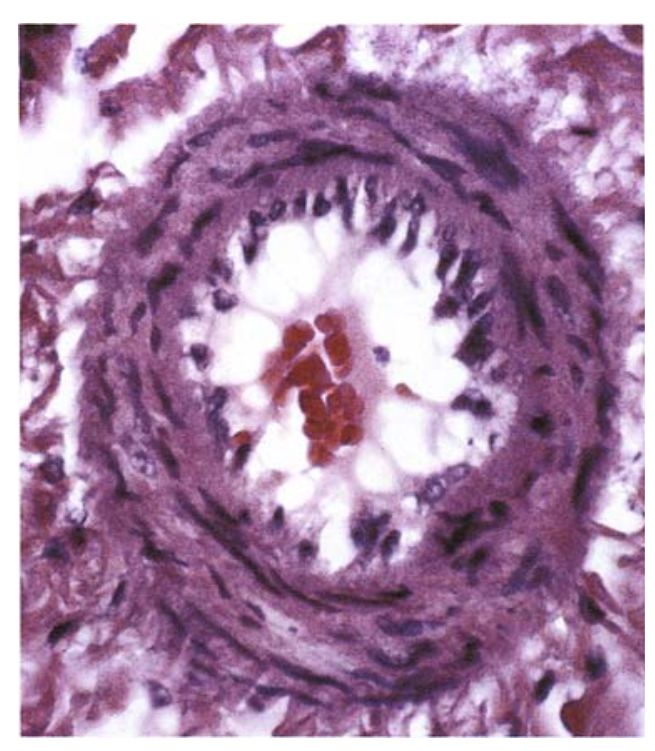

FigURE 31.16. Fat embolism. Clear, circumferential endoluminal vacuoles in muscular pulmonary artery. (Hematoxylin and eosin $[H \& E]$.) 


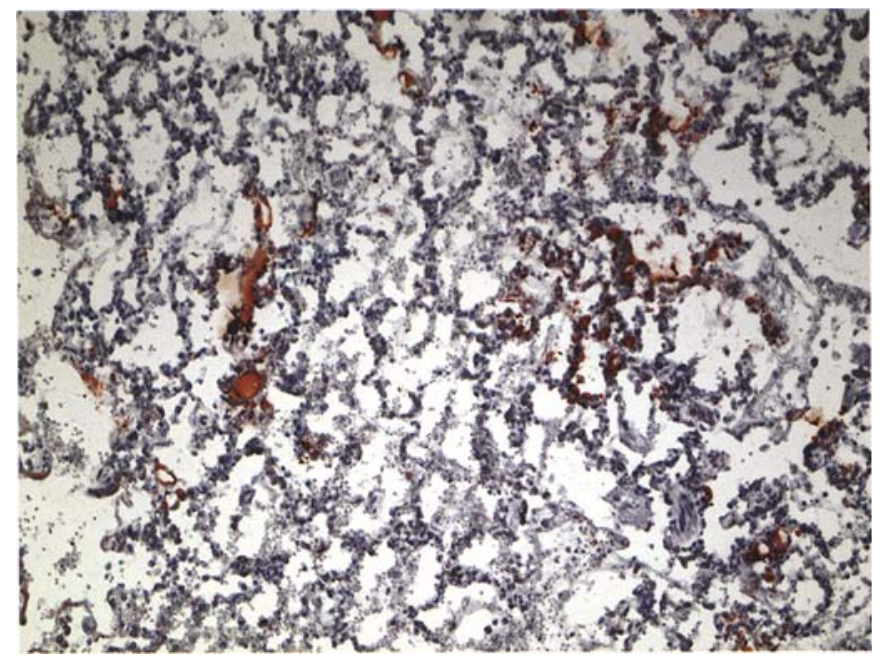

Figure 31.17. Fat embolism (oil red O).

Whereas the presence of PFE can be confirmed by demonstrating fat globules within the pulmonary vasculature, the diagnosis of FES requires clinical information. Most FES diagnostic criteria require involvement of at least two of the three major organ/tissue systems, as previously described. ${ }^{124,125}$ The diagnostic utility of seeing fat in bronchoalveolar cells obtained by BAL is unclear. ${ }^{125,148}$ Similarly, the diagnostic significance of seeing fat in pulmonary microvascular (PMV) cytology samples is not resolved. ${ }^{125,149-151}$

\section{Air Embolism}

Air embolism (AE), even though infrequently clinically significant, occurs in a variety of well-recognized scenarios. It is important to distinguish between venous air embolism and arterial air embolism because these two forms of AE have different routes of access to the circulatory system, pathologic findings, clinical manifestations, and therapeutic implications. ${ }^{125}$

Venous air embolism arises when air is introduced into the systemic venous circulation and is allowed to travel through the circulation to the right heart and then to the pulmonary arterial system. Rarely, it arises when air is introduced directly into the right ventricle or pulmonary artery. Venous air embolism has also been associated with rapid decompression. ${ }^{152}$ Clinically significant venous air embolism requires a relatively large amount of air. The effects of venous air embolism are primarily seen in the lungs. ${ }^{125}$

Arterial air embolism arises from air being introduced into the pulmonary venous circulation, then traveling to the left heart and into the systemic arterial circulation. On occasion, air in the systemic venous or pulmonary arterial systems may enter the systemic arterial system through a right to left shunt (e.g., patent foramen ovale or pulmonary arteriovenous malformation [AVM]) or by overwhelming the filtering capacity of the lung. ${ }^{125} \mathrm{~A}$ minuscule amount of air in the systemic arterial circulation may potentially have catastrophic effects. The effects of arterial air embolism are primarily observed in extrapulmonary organs, typically the heart and brain. ${ }^{125}$ The remainder of this section concentrates on venous air embolism.

The occurrence of venous air embolism requires the entry of air at some communication between a gas (usually atmospheric air) and an open venous system where the pressure gradient favors the ingress of gas into the systemic venous circulation. ${ }^{153}$ A pressure gradient favoring the ingress of gas can involve some combination of gas under positive pressure or subatmospheric venous pressure. A number of factors that increase negative intrathoracic pressure increase the likelihood of air embolism.

Venous air embolism is commonly iatrogenic and is recognized in a variety of clinical situations including neurosurgery, orthopedic surgery, head and neck surgery, obstetrics and gynecology, positive pressure ventilation, central venous catheter use and maintenance, and some radiographic procedures. ${ }^{125,153,154}$ Although venous air embolism is relatively common in some of these procedures (up to $40 \%$ of posterior cranial fossa neurosurgical procedures carried out on sitting patients), ${ }^{155,156}$ morbidity and mortality appear to be uncommon in modern times. Venous air embolism is also associated with trauma and orogenital sexual activity, ${ }^{157}$ the latter especially involving pregnant women when the vagina is orally inflated by exhaled air.

The significance of air in the venous system depends on the amount of air, the rate of infusion, the position of the patient, and the patient's cardiac status. Significant clinical dysfunction or death requires a large venous air embolism unless arterial air embolism supervenes. The minimal amount of air that needs to be infused to be lethal is not clearly established. However, assessment of multiple observed cases suggests that 200 to $300 \mathrm{~mL}$ of air rapidly infused is sufficient to cause the deaths of at least some persons. ${ }^{158-161}$ Others have opined that death can occur with the rapid infusion of as little as $100 \mathrm{~mL}$ of air. $^{162}$

The pathogenesis of dysfunction induced by venous air embolism is complex and involves a number of factors including mechanical obstruction to blood flow, pre- and postcapillary vasoconstriction, air/blood interactioninduced particulates, and pulmonary leukocytes. ${ }^{125}$

The clinical onset is typically sudden. It may be immediate or, less frequently, delayed. The symptoms and signs are for the most part nonspecific. Dyspnea is common. ${ }^{163}$ Other manifestations may include evidence of central nervous system dysfunction, fainting, light-headedness, chest pain, and anxiety. There may be evidence of hemo- 
dynamic compromise. The specific mill wheel murmur may be heard but, even in cases in which it is present, it tends to be transient. Instead, heart sounds are usually normal or include a harsh systolic murmur. ${ }^{125}$

Venous air embolism is recognized at autopsy by the presence of a large quantity of air in the undissected venous system, right heart (Fig. 31.18), or pulmonary artery. In clinically significant cases of AE, the blood in the right ventricle and pulmonary artery is typically frothy. Air bubbles may be seen along the route the air traveled from the ingress point to the right heart. Postmortem radiography may be useful in demonstrating the air (Fig. 31.19) ${ }^{164}$ Special dissection techniques may also facilitate the autopsy demonstration of AE. ${ }^{164}$

Baker ${ }^{165}$ has described a relatively simple dissection technique in which the heart is isolated and the coronary arteries, cardiac chambers, pulmonary artery, pulmonary veins, and vena cavae are sequentially opened in situ while submerged in water. ${ }^{164}$ Alternatively, entrapped air in the right ventricle may be demonstrated by the appearance of air bubbles entering a large airtight syringe half filled with water when the syringe needle is introduced into the right ventricle. ${ }^{166}$ Other techniques for collecting and measuring gas in the right ventricle have been described by Van Ieperen ${ }^{167}$ and reviewed by Bajanowski et al. ${ }^{168}$

Air embolism, venous or arterial, can be difficult or impossible to demonstrate after extensive resuscitative efforts, especially if open chest massage is employed. Similarly, air embolism cannot be anatomically demon-

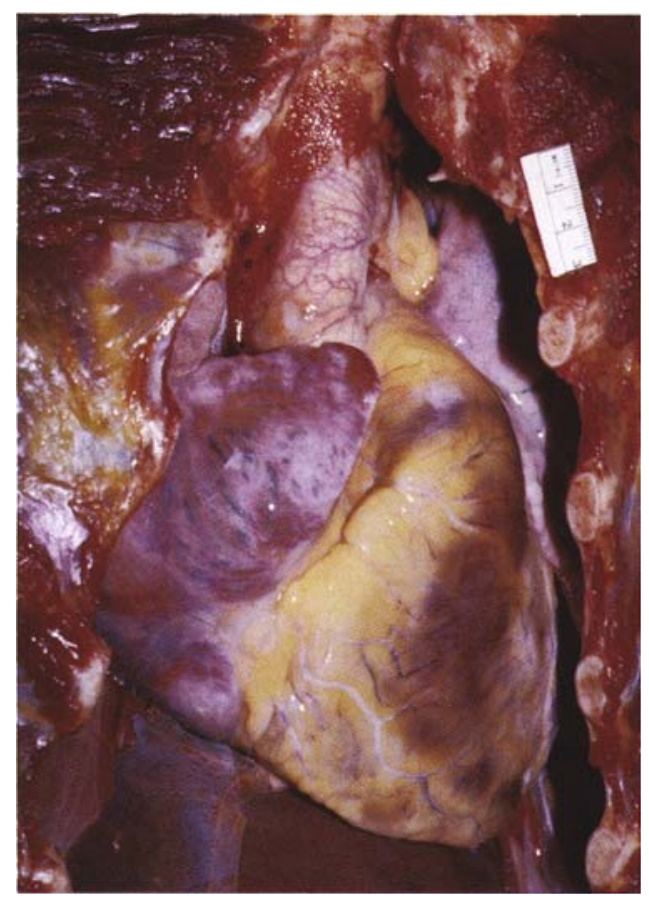

Figure 31.18. Air embolism. Right atrium distended by air.

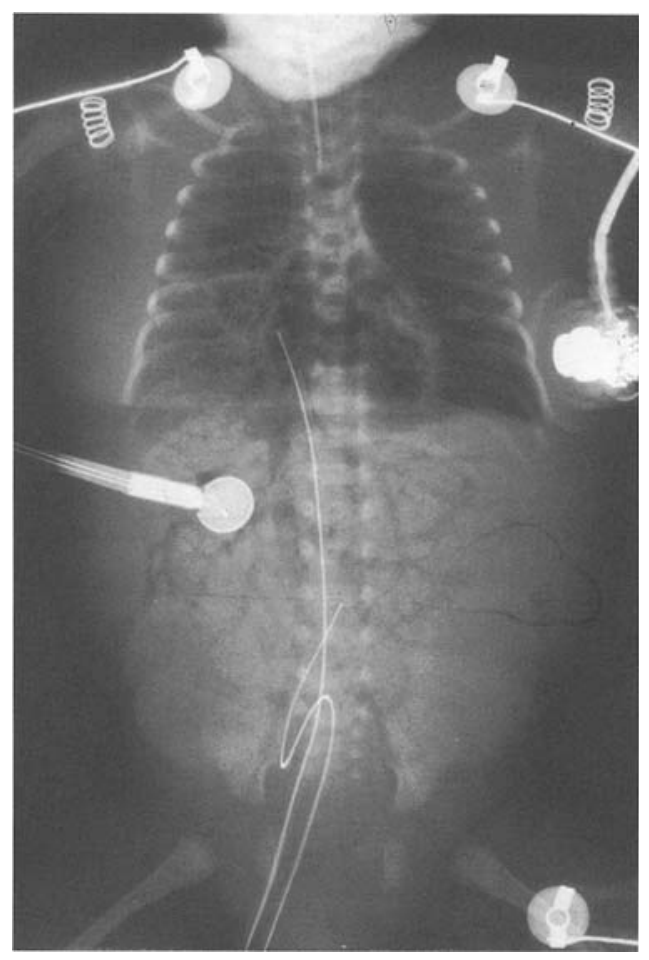

Figure 31.19. Air embolism. Chest radiograph showing hyperlucency due to air in the inferior vena caval system and right cardiac chambers.

strated in cases with prolonged postembolism survival or in cases with postmortem gas production due to decomposition.

\section{Amniotic Fluid}

Amniotic fluid embolism is an entity unique to pregnancy. The discussion of this entity follows (see Pregnancy).

\section{Tissue}

A variety of extrapulmonary tissues have been reported to embolize to the lungs (see also Chapter 28).

The most common of these is bone marrow (Fig. 31.20). Bone marrow emboli (BME) have been seen in $25 \%$ of road traffic fatalities. ${ }^{169}$ Their occurrence is also correlated with CPR-related chest compressions. ${ }^{169} \mathrm{Rib}$ and sternal fractures are major factors in determining the incidence of BME. ${ }^{169}$ Bone marrow emboli resolve by fragmentation and organization. The resolution of these fragments involve endothelialization, subendothelial fibrosis, and fibrosis. Hemopoietic elements are lost during the first week. Overall, BME resolve more slowly than do comparable thromboemboli. ${ }^{170}$ Bone marrow emboli are incidental findings and rarely, if ever, have clinical significance. 


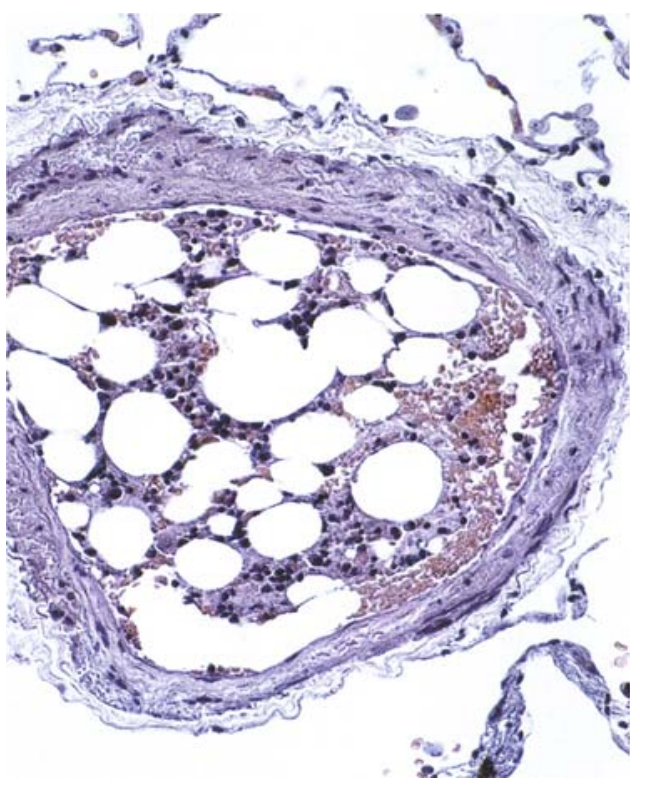

Figure 31.20. Bone marrow embolus.

Less commonly, tissue fragments from other sites may gain access to the systemic venous circulation and embolize to the lungs. Tissue embolization is most commonly associated with trauma, blunt or penetrating, during which time vascular channels and adjacent tissues are disrupted and fragmented. Brain (Fig. 31.21) and liver are the tissues most commonly observed in the pulmonary vasculature. ${ }^{171-175}$ Other tissues, including myocardium, have also been observed. These are usually incidental findings of no clinical significance.

Not all tissue emboli are the result of trauma. Brain emboli have been reported in cases of pediatric cerebral malformations. Liver tissue has been seen in instances of massive necrosis and in cases of neoplasia. Although most of these are also incidental findings, massive tumor emboli from hepatocellular carcinoma have resulted in sudden death (see Chapter 44). ${ }^{176}$

\section{Foreign Material}

Any insoluble particulate material that gains access to the systemic venous circulation may become lodged in the pulmonary microvasculature (see Chapter 28). By far, the most common situation leading to the deposition of significant amounts of material in this fashion is intravenous abuse of oral pharmaceutical preparations (see below, section on Substance Abuse and Chapter 26).

\section{Pneumothorax and Hemothorax}

Conditions associated with disruption of the pleura may result in hemothorax or pneumothorax. Most hemothora- ces related to blunt force injuries are the result of bleeding from lacerated lung, intercostal vessels, or internal mammary artery. Lung lacerations usually are associated with mild-moderate hemorrhage and often seal themselves spontaneously due to low pulmonary pressures and a high level of tissue thromboplastin. Occasionally, massive hemothorax may result from laceration of large pulmonary vessels. Massive hemothorax (at least $1500 \mathrm{~mL}$ ) may result in hemorrhagic shock, compression of the ipsilateral lung, and shift of the mediastinum. Massive hemothorax most often is the result of aortic laceration and is more common on the left side. ${ }^{177}$ The extent of bleeding from penetrating trauma is determined, in large part, by the size and site of vascular damage.

Pleuropulmonary or chest wall disruption may lead to the ingress of air into normally air-free areas-pleural space, mediastinum, soft tissue, pericardium, or the peritoneum. The most common clinically significant of these is the pleural space. Entry of air into the pleural space, pneumothorax, can result from several conditions, traumatic or nontraumatic.

Pneumothorax may arise spontaneously in the absence of underlying lung disease (primary) or as a complication of preexisting pleuropulmonary disease (secondary). ${ }^{178}$ Primary spontaneous pneumothorax typically occurs in men between the ages of 20 and 40 years. These men are often thin and tall. A history of smoking or substance abuse is often elicited. ${ }^{179-181}$ The pneumothorax is thought to be the result of rupture of a subpleural bulla or a pleural bleb, often precipitated by a Valsalva maneuver. The recurrence rate is variable but has been cited as $27 \%$ at 10 years. ${ }^{182}$

Secondary spontaneous pneumothorax usually arises in the setting of emphysema and occurs past the age of 35 years. Persons with Marfan's syndrome are also at increased risk for pneumothorax due to an increased incidence of apical blebs. ${ }^{179,181}$

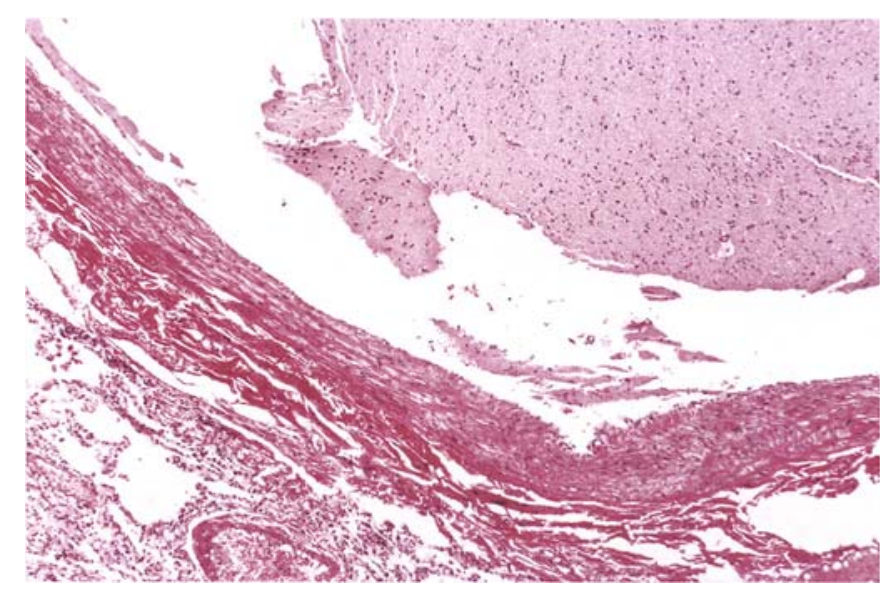

Figure 31.21. Brain tissue embolus. 


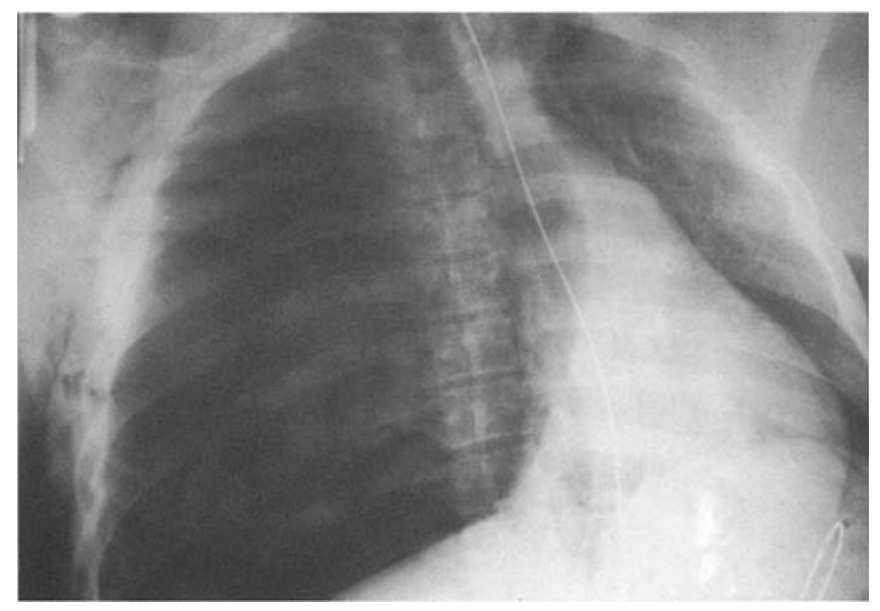

Figure 31.22. Tension pneumothorax with mediastinal shift towards left side (postmortem chest radiograph).

Pneumothorax may arise as the result of either blunt or penetrating trauma. In cases of blunt force injuries with rib fractures, the source of the air is generally a lung that has been punctured by a fractured segment of rib. ${ }^{179}$ Deceleration or barotrauma may be responsible for the development of pneumothorax in the absence of rib fracture. ${ }^{183}$ Some pneumothoraces are iatrogenic. Procedures most likely to result in production of a pneumothorax are thoracentesis, central vascular catheter insertion, and subacromial injection. ${ }^{179}$ The use of positive pressure ventilation may also lead to pneumothorax.

The clinical manifestations of pneumothorax depend on its size and type, and the clinical status of the patient. An uncomplicated simple pneumothorax is usually accompanied by shortness of breath and pleuritic chest pain $(80 \%$ to $90 \%)$ but may be asymptomatic $(10 \%){ }^{184}$ Respiratory distress generally becomes apparent in an otherwise healthy patient when at least $40 \%$ of the lung collapses. ${ }^{185}$

In some cases, a unidirectional air leak follows pulmonary disruption or chest wall perforation. Air enters the pleural space but cannot exit, resulting in progressively increasing intrathoracic pressure. The increased pressure may compress the lung, heart, great vessels, and trachea, and shift the mediastinum, resulting in impaired ventilation and decreased cardiac output. Chest wall defects leading to the development of a tension pneumothorax are often described as "sucking" chest wounds and the resultant pneumothorax as an "open" pneumothorax. An exception for the requirement of a one-way leak may arise during scuba diving. An otherwise simple pneumothorax may be converted to a tension pneumothorax as the gas expands during ascent. Clinical signs of a tension pneumothorax include asymmetric chest wall movement, distended neck veins, and tracheal deviation. Clinically, a pneumothorax may be identified on the basis of the history and physical findings but is most commonly assessed using standard chest radiography.

There are multiple ways to ascertain the presence of pneumothorax during autopsy. Postmortem radiography is useful in establishing the presence of pneumothorax, its extent, and the status of the mediastinum (Fig. 31.22). ${ }^{186}$ Pneumothorax can also be identified during the autopsy chest dissection. In most cases, a rush of air can be heard exiting the pleural space when the parietal pleura is broached. The detection of pneumothorax can be facilitated by relatively simple special dissection techniques. ${ }^{164}$ Pneumothorax can be identified by holding the dissected skin and chest wall soft tissue to form a pocket adjacent to the rib cage. The parietal pleura is incised after the pocket is filled with water. Escape of air bubbles through the pleural hole establishes the presence of pneumothorax. ${ }^{187}$ Apparatuses of various complexities, identical to those used in the detection and capture of air embolism, can also be used to detect, quantify, and analyze the content of pneumothoraces (see Air Embolism, above). ${ }^{164}$

\section{Pregnancy}

The incidence of maternal mortality in the United States is approximately $1 / 8000$ pregnancies or $1 / 10,000$ live births. It accounted for $0.099 \%$ of autopsies performed at the Dallas County Medical Examiner's Office during 1977 to $1999 .{ }^{188}$ Sudden death during pregnancy or in the postpartum period may involve a number of pulmonary causes of death that can be divided into a few broad categories (Table 31.2). One group of conditions can occur in the general population. These conditions may be unaffected by pregnancy, may have a propensity to occur during pregnancy (e.g., pulmonary thromboembolism due to deep venous thrombosis, air embolism), or may be accelerated or exacerbated by pregnancy (e.g., pulmonary hypertension, asthma). A second group consists of conditions unique to pregnancy and the puerperium (e.g., amniotic fluid embolism). Lastly, there is a group of conditions related to the care of the mother and fetus

TABLE 31.2. Pulmonary causes of death during pregnancy

1. Conditions unrelated to pregnancy

2. Conditions having propensity to occur during pregnancy

a. Deep venous thrombosis/pulmonary thromboembolism

b. Air embolism

3. Conditions exacerbated by pregnancy

a. Pulmonary hypertension

b. Asthma

4. Conditions unique to pregnancy and puerperium a. Amniotic fluid embolism

5. Conditions related to care of the mother or fetus

a. Anaphylaxis

b. Drug reactions and intoxications 
(e.g., anaphylaxis, intoxications, drug reactions), ${ }^{1,188}$ Among the various pregnancy-associated conditions that can cause death, some of the most common affect the pulmonary vasculature. ${ }^{189}$ Perhaps the most familiar of these to pathologists is thromboembolism complicating deep venous thrombosis.

\section{Deep Venous Thrombosis/Pulmonary Thromboembolism}

Deep venous thrombosis has been estimated to occur during the course of 1 to 3 of 1000 pregnancies. ${ }^{190}$ Due to difficulties in establishing the diagnosis among living patients, the true incidence may be significantly higher; $16 \%$ to $33 \%$ of DVT episodes occur during the first trimester. ${ }^{191}$ The risk of DVT is increased during pregnancy due to increased amounts of clotting factors and fibrinogen, placental production of fibrinolytic inhibitors, and, importantly, venous stasis. ${ }^{191-193}$ There is increased platelet activation, and protein $\mathrm{S}$ decline is proportional to the length of the gestation. ${ }^{191,194}$

Pulmonary thromboembolism remains one of the most common causes of maternal death, occurring in 4 to 13 of 10,000 pregnancies ${ }^{195}$ and accounting for approximately $9 \%$ to $12 \%$ of pregnancy-related deaths. ${ }^{196}$ Pulmonary thromboembolism occurs in $15 \%$ to $25 \%$ of pregnant patients with undiagnosed untreated DVT and is associated with $12 \%$ to $15 \%$ mortality. ${ }^{191,197}$ Death occurs within a half hour in two thirds of patients dying of PTE. ${ }^{192,198}$ The highest risk for PTE is during the immediate postpartum period. ${ }^{192}$

Increased risk factors for PTE among pregnant women include age greater than 35 years, two or more previous pregnancies, weight greater than 165 pounds, and a family history of DVT/PTE. Signs and symptoms of PTE during pregnancy include shortness of breath, tachypnea, cough, and pleuritic chest pain. Only $25 \%$ of women display the classic triad of dyspnea, pleuritic chest pain, and hemoptysis. ${ }^{199}$ Nearly all pregnant women with PTE will demonstrate decreased arterial $\mathrm{pO}_{2}$. However, one in six of these women will have a normal arterial $\mathrm{pO}_{2}{ }^{200}$

Pulmonary artery thromboembolism during pregnancy or in the postpartum period is morphologically identical to non-pregnancy-related thromboembolism and presents to the pathologist diagnostic and medicolegal issues similar to those already discussed (see above, and Chapter 28). Other entities causing pregnancy-related sudden death that typically affect the pulmonary circulation include pulmonary hypertension, amniotic fluid embolism, and air embolism.

\section{Primary Pulmonary Hypertension}

Primary pulmonary hypertension may be exacerbated by pregnancy. Whether pregnancy can cause PPH is unset- tled. ${ }^{201}$ Clearly, pulmonary hypertension and pregnancy are a lethal combination, with mortality being approximately $40 \%$ to $50 \% .{ }^{201-206}$ Most of the deaths occur during the third trimester or early postpartum period. Cardiovascular collapse occurring immediately postpartum after a supine position delivery may be related to the sudden increase in venous return to the right heart as a consequence of infusion of uterine blood into the circulation and decompression of the inferior vena cava in the face of fixed elevated pulmonary resistance. ${ }^{1}$ The morphology of pulmonary hypertension in the pregnant woman has the same features as pulmonary hypertension seen in the general population (see Chapter 28).

\section{Amniotic Fluid Embolism}

Amniotic fluid embolism (AFE) was initially described by Meyer $^{207}$ in 1926 and was established as a distinct entity by Steiner and Lushbaugh ${ }^{208}$ in 1941. It is a relatively rare condition that affects 1 in 8000 to 1 in 80,000 pregnancies. ${ }^{209-211}$ Although uncommon, it is still a leading cause of maternal mortality and morbidity in the developed world, ${ }^{212}$ accounting for $7.5 \%$ to $10 \%$ of maternal deaths in the United States, Australia, and the United Kingdom, ${ }^{196,213}$ a percentage of deaths that has not significantly changed in nearly 25 years. ${ }^{214,215}$ Mortality has been reported to be high in recognized cases; however, the incidence of subclinical or milder cases of AFE is not well defined. As recently as 1979 , mortality was reported as $86 \% .{ }^{210}$ A 1995 report from the U.S. suggested $61 \%$ mortality, with $85 \%$ of survivors having neurologic impairment. ${ }^{216}$ Later data suggest a less than $30 \%$ mortality. ${ }^{212,216-218}$ The apparent drop in mortality probably represents improvement in therapy and the recognition of milder cases.

Amniotic fluid embolism typically presents during labor $(70 \% \text { of cases })^{219}$ or shortly thereafter. Rare cases have been reported to occur in the late postpartum period. Amniotic fluid embolism has also been associated with cesarean section ( $19 \%$ of cases) ${ }^{219}$ abortion, abdominal trauma, uterine rupture, and intrapartum amnioinfusion. ${ }^{220}$ Risk factors for AFE include advanced maternal age, multiparity, and tumultuous labor. ${ }^{188}$ The clinical syndrome is typically heralded by the acute onset of shortness of breath and hypotension followed by cardiopulmonary collapse. ${ }^{219-221}$ Other frequent signs and symptoms include disseminated intravascular coagulation (DIC), pulmonary edema, seizures, and fetal distress. ${ }^{125}$ In fatal cases, death usually occurs within hours of collapse and, in many cases, within 1 hour of the onset of symptoms. ${ }^{125,212,222}$ The development of AFE requires ruptured gestational membranes, a pressure gradient favoring movement from the uterus to the maternal circulation, and an entry site for the amniotic fluid to reach the cir- 
culation. ${ }^{125,220,223}$ The latter is usually through small tears in the lower uterine segment or endocervix. ${ }^{223-225}$

Complex pathophysiologic alterations induced by the constituents of amniotic fluid are responsible for the perturbations of cardiovascular function, oxygenation, and coagulation that characterize AFE. ${ }^{20,226}$ These pathophysiologic mechanisms have not been fully clarified, but it appears that the primary process is not simply mechanical obstruction of the pulmonary vasculature by the solid material in the amniotic fluid. ${ }^{125}$ The amount of particulate matter in the pulmonary vasculature in cases of AFE does not always correlate with the clinical course, a feature of importance to the pathologist, as will be discussed subsequently. It has been postulated that AFE involves a complex immunohumoral reaction resulting from exposure to fetal products that incites an immunologic response in the mother leading to the observed clinical syndrome. ${ }^{219,221} \mathrm{~A}$ role for anaphylaxis in AFE has been suggested by a number of cases in which elevated blood tryptase concentration has been measured. However, a number of other cases with decreased complement and normal tryptase concentration suggests a role for complement activation rather than mast cell degranulation in the development of the clinical syndrome of AFE. ${ }^{227-230}$

Experimental and human observations support a biphasic hemodynamic phenomenon. ${ }^{221,226,231}$ The initial response is suggested to be acute pulmonary vasoconstriction that may lead to right heart failure. This phase is transient and is postulated to be replaced by the effects of the release of inflammatory materials in the lung that induce a capillary leak syndrome and depression of the myocardium. The initial vasoconstrictive phase has not been observed in humans but has been documented in experimental animals. The lack of documentation of the initial phase in humans may reflect the lack of hemodynamic monitoring during the early phase in human cases. ${ }^{221}$

Classically, the definitive diagnosis of AFE has been made at autopsy and requires demonstrating the presence of amniotic fluid in maternal pulmonary blood vessels. $^{222}$ The presence of identifiable amniotic fluid within maternal lung tissue sections is almost always, if not always, abnormal. An AFE is not a common physiologic occurrence ${ }^{220}$; contraction of the uterus appears to be effective in collapsing uterine veins.

Amniotic fluid is generally identified by recognizing fetal debris in the maternal pulmonary arterioles and capillaries (Fig. 31.23). Special stains and techniques are often, although not always, necessary to document the presence of these materials, such as lanugo hair, fetal squames, lipid, and mucin. ${ }^{125}$ Polarized light may help identify the presence of fetal lanugo within the maternal pulmonary circulation. Combination stains, such as the Attwood (Fig. 31.23B) and Movat stains, will differentially stain a variety of amniotic fluid components. ${ }^{232}$ Immunohistochemical staining may also be helpful in establishing the presence of amniotic fluid especially early in pregnancy, when the volume of amniotic fluid is small, as is the amount of fetal debris within the fluid. TKH-2 (monoclonal antibody directed to sialyl Tn, NeuAc $\alpha$ 2-6 GalNAc) immunostaining has been reported to be a sensitive method to detect mucin derived from meconium and amniotic fluid. ${ }^{233}$ Squamous cells may be identified by staining for high molecular weight cytokeratin (HMW-CK), which does not stain pulmonary capillary endothelium (Fig. 31.24).

There is no routine diagnostic scheme to confirm the presence of AFE in the living patient. Rather, it is a
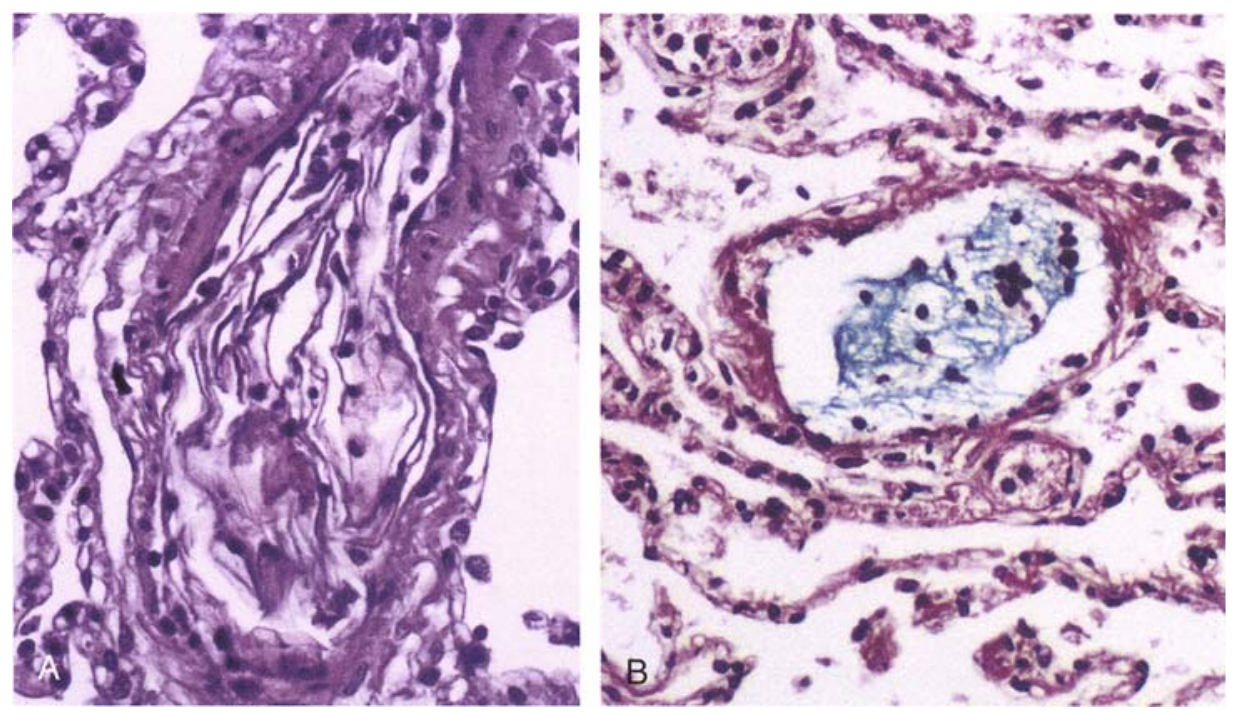

Figure 31.23. Amniotic fluid embolism. A. Amniotic squames in a muscular pulmonary artery. (H\&E.) B. Attwood stain showing intravascular mucinous material. 


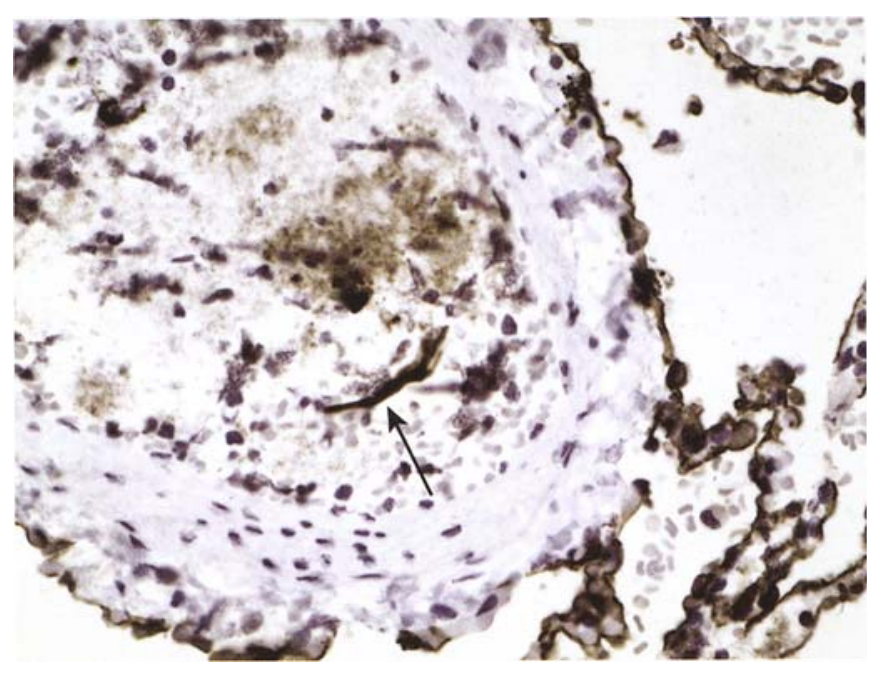

FIgURE 31.24. Amniotic fluid embolism. Immunostain for cytokeratin demonstrating intravascular squames (arrow). Alveolar epithelium is also cytokeratin positive (right lower corner).

diagnosis of exclusion. Rarely, open lung biopsy has been used to establish the diagnosis. ${ }^{234}$ Fetal squames have reportedly been seen in a sputum cytology prepared from a woman with AFE. ${ }^{235}$ The observation of squamous cells in PMV cytology samples has also been put forward as a diagnostic modality useful in diagnosing AFE. ${ }^{236}$ However, some authors have considered the observation of fetal squames in PMV samples neither specific nor sensitive for the diagnosis of AFE. ${ }^{222}$

Squamous cells and other fetal debris have been reportedly found in PMV cytology samples in some patients without clinical evidence of AFE who were undergoing hemodynamic monitoring during delivery. ${ }^{125}$ Isolated fetal squamous cells have been occasionally recognized in maternal blood in cases with no clinical evidence of AFE; however, in these cases the squames were not accompanied by mucin, lanugo, or vernix. ${ }^{220}$ It is difficult to reliably differentiate adult from fetal squames, and the squamous cells seen in the maternal blood during PMV studies are thought to represent specimen contamination or cells derived from the entry site of the pulmonary artery catheter.

Davies $^{220}$, in a 2001 review, supports the use of the technique developed by Masson for obtaining a PMV sample through the distal lumen of a wedged pediatric anesthesia catheter ${ }^{223}$ Masson $^{223}$ has concluded that the observation of large numbers of squamous cells in such a sample strongly supports the diagnosis of AFE, particularly when the squamous cells are coated with neutrophils or if they are accompanied by other fetal debris. It has also been suggested that confirmation of the diagnosis might center on the identification of other amniotic fluid elements rather than the squamous cells. $^{237}$
The identification of trophoblastic cells in the maternal circulation is of no diagnostic utility in AFE because these cells are normally free in the intervillous space and thus have direct access to the maternal circulation. Attwood and Park, in a histopathologic study of the lungs of women dying in the peripartum period, were able to identify trophoblastic emboli in $43 \%$ of the cases while demonstrating AFE in less than 1\% (see also Chapter 28 and Fig. 28.39). ${ }^{238}$

Currently, noninvasive tests shown to be reliable in the diagnosis of AFE are lacking. The demonstration of elevated blood serum concentrations of sialyl Tn antigen and plasma concentrations of zinc coproporphyrin have been posited as being promising tests for diagnosing AFE. Clinical experience with both of these tests is limited and further studies are needed in order to assess their utility and reliability. ${ }^{220}$

\section{Air Embolism}

Air embolism accounts for approximately $1 \%$ of maternal deaths. It may complicate normal labor, delivery with placenta previa, criminal abortion, gynecologic procedures involving vaginal insufflation and sexual activity, ${ }^{239}$ most commonly orogenital. ${ }^{240-242}$ The air typically enters the venous circulation through the subplacental sinuses or other uterogenital veins. ${ }^{188}$

\section{Asthma}

The prevalence of asthma among pregnant women has been reported to be $1 \%$ to $8.4 \% .{ }^{243,244}$ The clinical status of asthma during pregnancy is variable. ${ }^{245}$ Overall, $22 \%$ to $27 \%$ of women report improvement in their asthma, $26 \%$ to $40 \%$ indicate no change in their condition, and $20 \%$ to $42 \%$ of women report some worsening of their asthma. ${ }^{246-248}$ Among 366 pregnancies followed by objective criteria, asthma improved in $28 \%$, was unchanged in $33 \%$, and worsened in $35 \% .{ }^{249}$ The medical aspects of the pregnant asthmatic and vice versa have been extensively reviewed by Guy et al. ${ }^{250}$

The pathologic appearance of asthma during pregnancy is the same as in the nonpregnant population and has been discussed previously in this chapter and in Chapter 15.

\section{Trophoblastic Tissue}

Embolization of trophoblastic tissue including chorionic villi, syncytiotrophoblasts, and cytotrophoblasts is a rare cause of sudden unexpected death that is typically associated with surgery or other instrumentation (see Fig. 28.39 in Chapter 28). ${ }^{251}$ Placenta accreta may be associated with a higher incidence of this disorder. ${ }^{251}$ Extensive embolization of chorionic villi has also been attributed to abdominopelvic blunt trauma. ${ }^{252}$ 

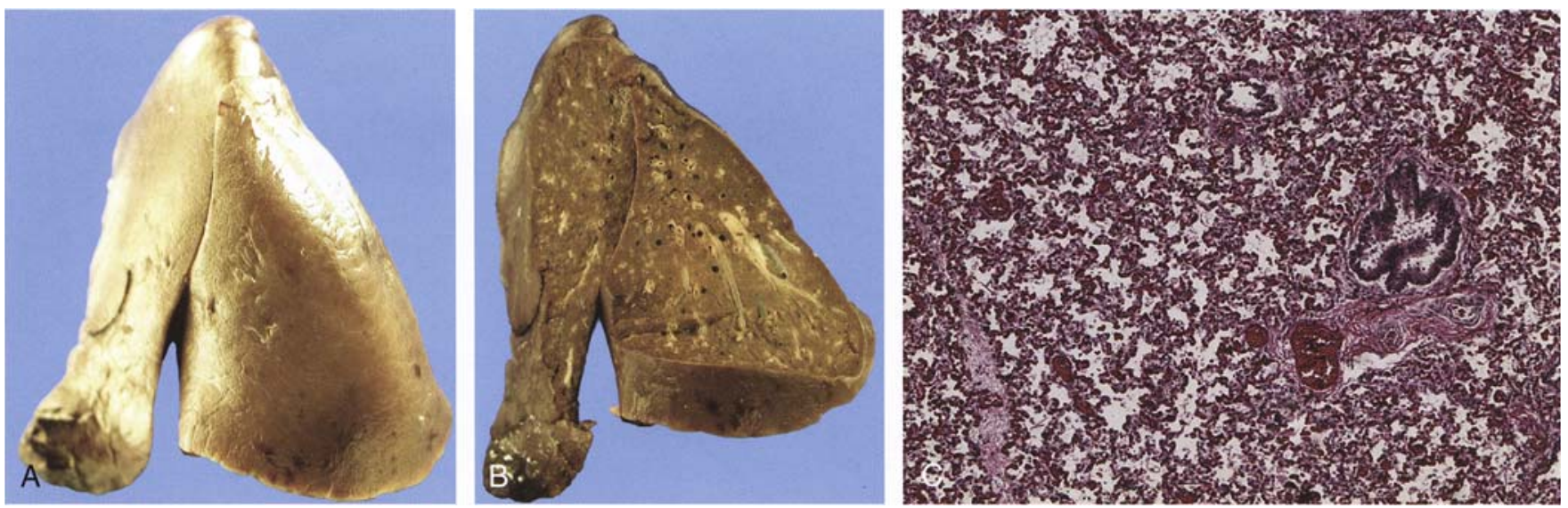

Figure 31.25. Stillborn lung. A,B. Lung has a solid, dark, liver-like appearance. C. Alveolar septa are congested and incompletely expanded.

\section{Pediatric Issues}

\section{Live Birth Versus Stillbirth}

A major issue facing the pathologist during the evaluation of a dead neonate is whether or not the infant was liveborn. If livebirth is not demonstrable, it is generally presumed that the child was stillborn. A number of factors may be considered during this assessment. In some cases changes indicative of intrauterine demise will be present. Witness accounts of crying or other postnatal activities may be useful in establishing live birth. Factors considered in whether live birth occurred also may include such things as evidence of breathing, food in the stomach, inflammatory response in the umbilical stump, and air in the middle ears.

Inflammation in the umbilical stump and food in the stomach are the most definitive objective evidence of livebirth. Aeration and expansion of the lungs are frequently used in attempting to assess whether or not the infant breathed air. The extent of aeration will be dependent on the length of time the infant breathed and the depth of the breaths. The ability to reliably demonstrate that breathing occurred may be problematic in some cases.

Careful gross examination of the lungs is probably as good a method as any to assess pulmonary aeration. The lungs of a stillborn infant are typically dark red, small, rubbery, and, on cut section, have a uniform almost liverlike appearance (Fig. 31.25). In contrast, the lungs of a child who has breathed normally, even for a short period of time, are characteristically well expanded, salmonpink, soft, and spongy (Fig. 31.26).

Over the years various tests have been proposed to help assess aeration. Historically, the method used most often to assess whether breathing has occurred is the
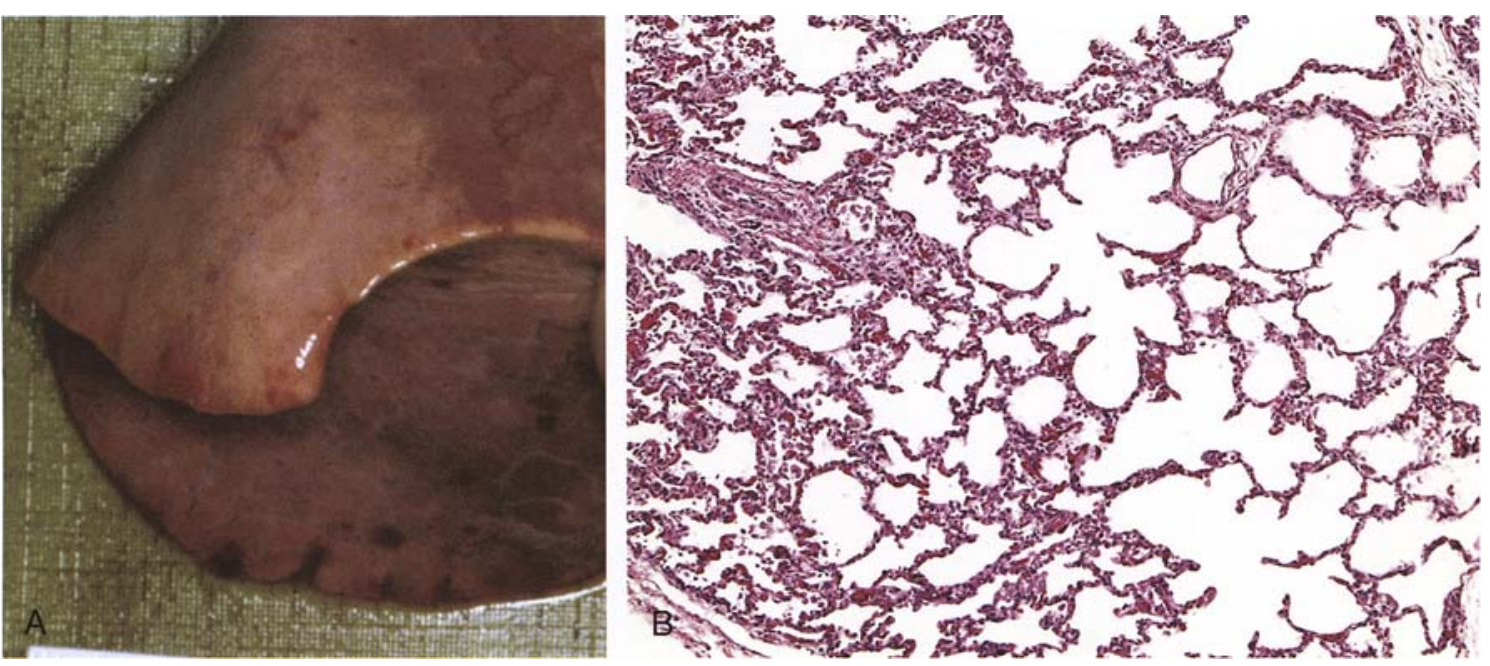

Figure 31.26. Liveborn lung. A. Pleural surface is pink and smooth. B. Focally, alveoli appear fully expanded. 
hydrostatic test. This test is performed by simply placing the lungs in a container of water and observing whether or not they float. Various modifications of the test specify using one intact lung, both lungs, or both lungs with the adjacent mediastinal structures attached. Flotation implies aeration and thus breathing, whereas lungs that sink are considered not to have been aerated and are thus indicative of stillbirth. Unfortunately, this test is fraught with false-positive and false-negative results and is thus of limited use. ${ }^{253}$

Gas generated during decomposition or air forced into the lungs of a stillborn during resuscitation attempts can result in lung flotation and a false-positive flotation test. Infants born alive who carry out feeble breathing efforts may not aerate their lungs to the extent that the lungs float and will provide a false-negative hydrostatic test. A number of attempts have been made to establish histologic criteria for establishing the presence of breathing. ${ }^{253}$ Overall, these are not particularly helpful and probably do not add anything to a well-performed gross inspection. Overall, fully expanded lungs that float in water in the absence of putrefaction and resuscitation strongly support live birth. Similarly, unaerated lungs as described above that sink in water strongly support stillbirth. In cases with intermediate findings the determination of livebirth or stillbirth should rest primarily on other factors. As so aptly stated by Adelson ${ }^{254}$ more than 25 years ago, unless pathologists have incontrovertible evidence of postnatal survival, they are bound not to diagnose livebirth.

\section{Sudden Infant Death Syndrome}

Sudden infant death syndrome (SIDS) is currently defined as "the sudden death of any infant under the age of 1 year, which remains unexplained after a thorough case investigation, including performance of a complete autopsy, examination of the death scene, and review of the clinical history." 255 Subsequent to the formulation of this definition, there have been a number of proposed modifications. ${ }^{256}$ These offer some refinements; however, the major features and requirements for the diagnosis remain basically the same. Protocols for autopsy and investigation of sudden, unexpected infant deaths are available. ${ }^{255}$

There is marked variation in the incidence of SIDS among different populations, both around the world and within individual societies. SIDS is the leading "cause" of unexpected death of infants between 1 month and 1 year of age. SIDS deaths most commonly occur between 2 and 4 months of age, are rare after 8 months and are characteristically associated with sleep. ${ }^{257,258}$ Currently, approximately $20 \%$ of cases occur while the child is in the custody of a child-care provider. ${ }^{259}$ Prior to 1992, the reported incidence of SIDS in the United States was approximately 1.5/1000 live births.
Although the term SIDS imparts an impression of a recognized disease entity with distinct pathogenesis, the reality is that SIDS is a diagnosis of exclusion and likely represents the end point of a number of conditions characterized by having no currently recognizable morphologic or laboratory definitive diagnostic markers. It has been described as a likely "complex amalgam of predisposing factors, external stressors, and underlying vulnerabilities." ${ }^{260}$ Nevertheless, the relative uniformity in the epidemiologic and pathologic features of these deaths supports that there are features common to many of them.

Studies have primarily centered on the respiratory and central nervous systems as being the critical locations of "defects" resulting in SIDS. Over the years, of the dozens of theories that have been proposed to explain SIDS, most have fallen by the wayside. A number of studies have reported changes thought to be related to chronic hypoxia. ${ }^{261,262}$ Among these proposed hypoxia markers was reported hyperplasia and hypertrophy of the media of pulmonary vessels. Other studies have failed to confirm most of these observations. ${ }^{263-266}$ Most recently, normal developmental modifications of arousal and autonomic function and how they may interact with environmental stressors are being carefully explored and appear to offer promise in explaining a part of the mechanism leading to the deaths of many of these infants. ${ }^{1267,268}$

One of the strong risk factors for SIDS that has been extensively studied is prone sleeping (especially when it is not the accustomed position) and, by extension, the overall sleep environment. Cote et al. ${ }^{269}$ have reported that 139 of 157 SIDS cases were found prone, although only 93 of the infants usually slept prone. In a series of SIDS deaths in Chicago, the odds ratio (OR) for prone sleeping was 2.4. In addition, the OR was 2.5 for infants sleeping on soft bedding or whose heads/faces were covered by bedding. However, the OR was 21 for those infants dying prone on soft bedding. ${ }^{270} \mathrm{~A}$ mechanism of death in these infants has been proposed as rebreathing of exhaled air. ${ }^{271}$ Coupled with being in this potentially compromising position is the possibility of increased vulnerability of the child, notably developmental changes in the brainstem nuclei involved in reflex responses to cardiorespiratory stimuli,including arousal. ${ }^{267,268,272}$ Depressed arousal from sleep has been linked to a variety of recognized SIDS risk factors including recent illness, prone sleeping, maternal smoking, and prematurity. Increased arousability has been linked to factors associated with a decreased risk of SIDS such as the use of pacifiers and breast-feeding. ${ }^{273,274}$

Successful modification of these risk factors, particularly in avoiding prone sleeping and soft bedding, avoiding passive smoking, and promoting breast-feeding (Back to Sleep campaign $)^{275}$ has been associated with a reported $44 \%$ decrease in the incidence of SIDS in the United States. ${ }^{276}$ 


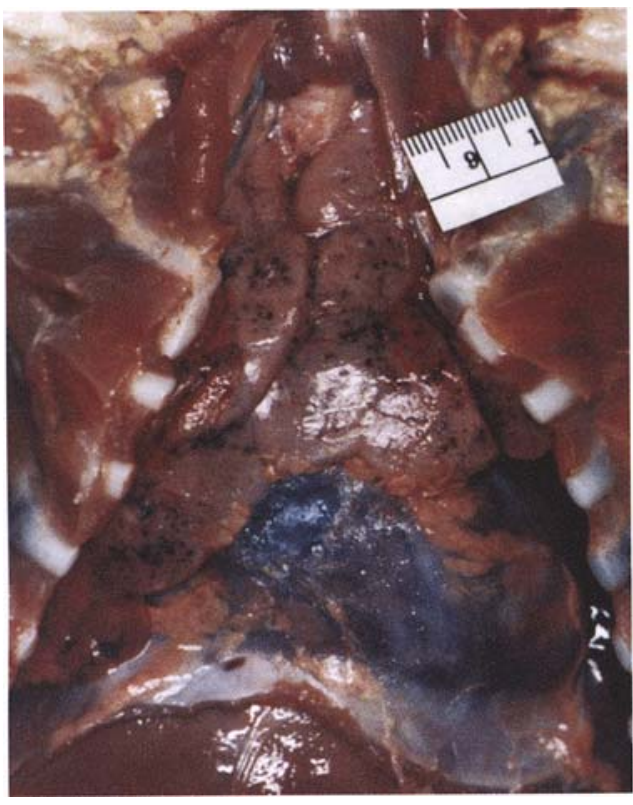

FIGURE 31.27. Sudden infant death syndrome. Thymic petechiae.

Although a dramatic decrease in SIDS has been reported from multiple populations that have had campaigns to change sleep position and other SIDS risk factors, it is not clear how much of the apparent drop in SIDS is due to diagnostic shift from SIDS to suffocation or "undetermined," and how much is a true decrease in the number of infant deaths. A recent study based on review of death certificate data suggests that up to $90 \%$ of the apparent decrease in SIDS may be due to a shift in death certification practices. ${ }^{277}$ More definitive answers should become available as detailed studies of specific populations are reported.

As noted previously, autopsy findings alone are not diagnostic of SIDS. The autopsy (including appropriate laboratory studies such as toxicology and metabolic disorder screening) primarily excludes the presence of recognizable diseases or injuries sufficient to cause death.

Typical postmortem findings in SIDS infants include thymic petechiae (50\% to $85 \%$ of cases) (Fig. 31.27). ${ }^{278-280}$ Pleural and epicardial petechiae are also common. The lungs are typically congested and edematous. The congestion may be associated with focal extravasation. There is often some formula or curdled milk in the stomach reflecting the onset of the lethal incident shortly after being put down to sleep following feeding.

Microscopically, focal mild lymphocytic interstitial infiltrates are commonly seen in the lungs of infants whose deaths were attributed to SIDS. Occasionally, the infiltrates are more extensive. Krous et al. ${ }^{281}$ reported that the incidence and extent of these infiltrates did not differ between SIDS and control groups. They opine that minor respiratory inflammatory infiltrates are neither a cause of sudden infant death or a specific marker for lethal respiratory infection in infants dying suddenly and unexpectedly. Contrary to the history obtained in SIDS cases, interstitial pneumonitis or bronchiolitis sufficiently severe to cause death is preceded by clinical illness with signs of lethargy, tachypnea, respiratory distress, feeding difficulty, or apnea. ${ }^{281}$

In many SIDS infants, there may be pulmonary edema fluid/froth or unclotted blood coming from the nose or mouth. These reflect agonal pulmonary edema or leakage from engorged nasopharyngeal small blood vessels, and do not have the serious implication of suffocation that inexplicable blood from the nose of a living infant carries. $^{282}$

\section{Blunt Force and Penetrating Injuries}

Injuries affecting pulmonary function may involve the lungs, extrapulmonary airway, or other extrapulmonary structures. The injuries may result from either blunt or penetrating trauma, the former being more common. Chest injuries are responsible for $20 \%$ to $25 \%$ of traumatic deaths and may contribute to a similar number of deaths in the United States. ${ }^{185}$ Significant blunt chest injuries are most frequently the result of motor vehicle accidents. $^{283}$ Overall, there is a $7 \%$ chance of sustaining significant chest trauma in any random motor vehicle crash. ${ }^{284,285}$ Other causes of significant blunt force thoracic injury includes falls from height, work or recreational crush injuries, and assaults. ${ }^{286}$

Penetrating injuries, accounting for about one third of hospital trauma cases ${ }^{287}$ are commonly due to stab wounds and firearm injuries (Fig. 31.28). Stab wounds

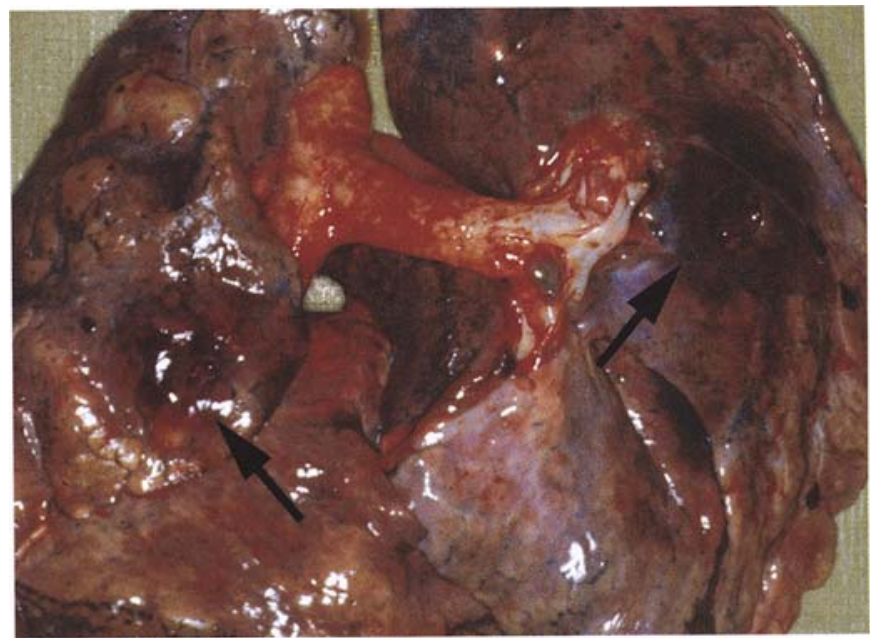

FIGURE 31.28. Gunshots (two) of the lungs. Each central defect is surrounded by a zone of hemorrhage (arrows). 
tend to predominate in hospital series, whereas firearm injuries are more common in medical examiner office series due to the higher mortality associated with the latter. In patients arriving at the hospital alive, stab wound mortality is less than $5 \%$ while gunshot mortality is $10 \%$ to $20 \% .{ }^{288}$ Most deaths caused by chest injury involve damage to the heart or great vessels. Isolated respiratory tract injury occasionally causes death but is more likely to lead to morbidity.

Thoracic blunt force damage results from compressive, shear, or blast forces (see Explosions, below, for a discussion of blast injury). ${ }^{283}$ The chest wall, lungs, and extrapulmonary airway may be damaged. One of the most common thoracic injuries sustained as a result of blunt force is rib fracture. ${ }^{289} \mathrm{Rib}$ fracture may result from either direct impact or thoracic compression. It is more common in adults, especially the elderly, due to decreased rib cage flexibility. Osteoporosis increases the susceptibility to fracture.

Single nondisplaced rib fractures are usually of little physiologic significance in otherwise healthy persons; however, in the elderly or in persons with significant comorbid conditions they may contribute to morbidity and mortality. Displaced rib fractures may be associated with disruption of adjacent structures such as intercostal vessels and pleura. Fragments of rib can penetrate the lung, heart, and great vessels. Multiple rib fractures result in increased pain and the possibility of ventilatory impairment, and increase the likelihood of coexisting serious injury. The most serious blunt chest wall injury is flail chest, a clinical condition characterized by paradoxical chest movement with breathing. ${ }^{283}$ Flail chest is present in $5 \%$ to $13 \%$ of chest injury patients and is associated with increased mortality. ${ }^{287}$ Flail chest results from isolation of a segment of the chest wall by rib fractures characteristically involving the anterior or lateral aspects of at least three to four consecutive ribs in at least two places ${ }^{283}$ costochondral separation, sternal fracture, or a combination of these fractures.

Clinically significant respiratory impairment may be the result of flail chest. Age and comorbid conditions are important factors in determining the clinical effect of the flail segment. The onset of impairment may be delayed. The paradoxical movement of the flail segment may be masked for several hours by intercostal muscle splinting. ${ }^{283}$ Posterior rib fractures often do not lead to flail chest because of effective splinting by the thick musculature of the back. The major physiologic consequence in flail chest has been attributed to contusion of the adjacent lung and decreased vital capacity. ${ }^{290}$

Lung contusion (bruise) is the most common lung injury caused by blunt force and is also the most common potentially lethal chest injury. Like bruises elsewhere on the body, pulmonary contusion results primarily from the tearing of parenchymal small blood vessels. These tears

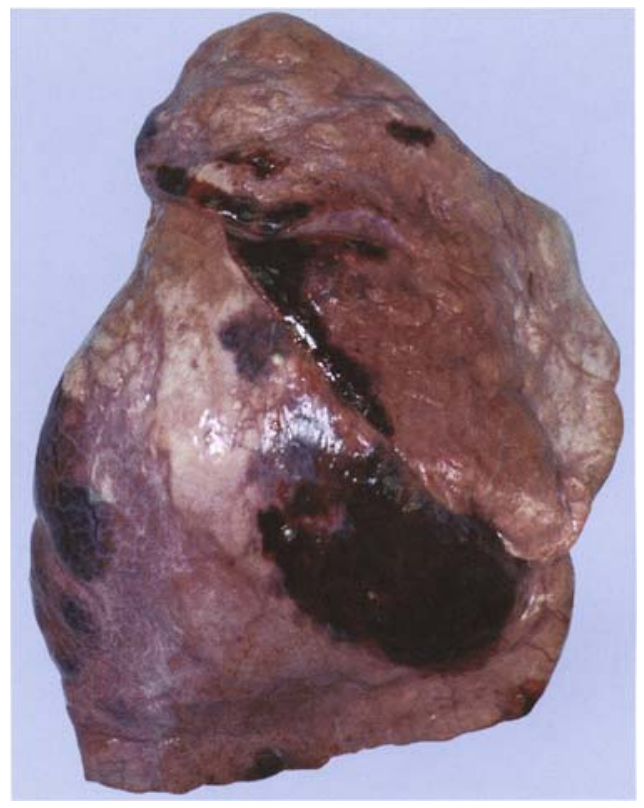

Figure 31.29. Pulmonary contusion.

are not grossly apparent. The resulting bleeding leads to a discrete area of parenchymal hemorrhage with most of the extravasated blood being found within air spaces (Fig. 31.29). In adults, the presence of pulmonary contusion is more likely if ribs are fractured. However, significant lung contusion or other serious intrathoracic injuries can occur in the absence of rib fractures, notably when the ribs are flexible such as in children and young adults.

Lung contusion also serves as a marker for other serious injuries, some of which may be extrathoracic. Some other intrathoracic injury is present in $87 \%$ of patients with motor vehicle crash-related pulmonary contusion. ${ }^{291}$ One study reported extrathoracic injuries in $67 \%$ of patients with either pulmonary contusion or flail chest but in $92 \%$ of patients who have sustained flail chest and pulmonary contusion. ${ }^{292}$

The extent and rapidity of development of lung contusion is dependent on the extent of vascular disruption and the extent and rate of bleeding. Grossly, contusion is visible almost immediately. However, contusion may not be evident on a chest radiograph for several hours. Contusions resolve quickly. Clearing is seen radiographically within 1 to 2 days, with resolution in 3 days. ${ }^{293,294}$

Pulmonary contusion manifests itself by the development of respiratory failure over time. The degree of respiratory impairment caused by pulmonary contusion is proportional to the extent of the contusion. Acute respiratory distress syndrome (ARDS) develops in $82 \%$ of patients having more than $20 \%$ of their lung tissue contused, whereas only $22 \%$ develop ARDS if less than $20 \%$ of the lung is bruised. ${ }^{283}$ The extent of preexisting lung disease is also a determinant factor in the clinical signifi- 


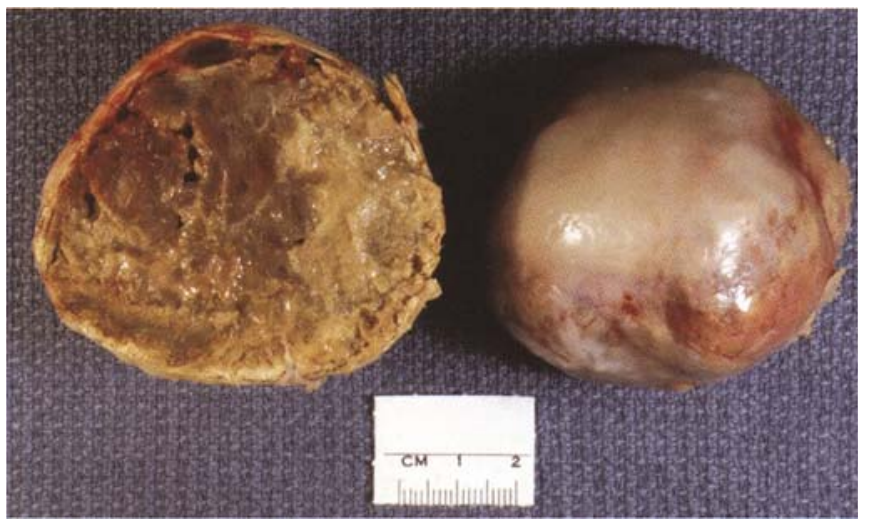

FIGURE 31.30. Chronic pulmonary hematoma/contusion presenting as a solitary lung mass. Resected encapsulated lesion includes necrotic lung and degenerated blood clot.

cance of pulmonary contusion. The extent of pulmonary contusion also correlates with the subsequent development of pneumonia. ${ }^{283}$

Most patients recover from pulmonary contusion, although the recovery may be prolonged. Less than $5 \%$ of surviving patients are pulmonary disabled on the basis of pulmonary contusion. ${ }^{283}$ The mortality associated with either pulmonary contusion or flail chest is $16 \%$. However, mortality is $42 \%$ when both of these conditions are manifest. ${ }^{292}$

More severe blunt force, usually due to sudden compression or shearing, may result in more extensive disruption of the lung tissue than is evident grossly and is best described as laceration. Leakage of air and bleeding into the damaged area leads to the development of a hematoma that may also contain air. Pulmonary hematomas are more common in children and young adults due to the increased flexibility of the chest wall.

Most lung hematomas undergo progressive resolution. Although shrinkage of the hematoma is usually evident by 6 weeks, the hematoma may persist radiographically for up to 4 months. ${ }^{294}$ Exceptionally, the walled-off hematoma may persist longer (Fig. 31.30). ${ }^{295}$

Severe blunt force, especially to the lower chest in children, may result in torsion of the lung. The lung is displaced 180 degrees resulting in the lung apex abutting the diaphragm (see Chapter 5). Severe blunt force to the lower anterior chest is also associated with rupture of the diaphragm, a condition found in $0.8 \%$ to $1.6 \%$ of blunt force injury patients. ${ }^{185}$ Although most often caused by blunt force $(75 \%)$, penetrating trauma can also disrupt the diaphragm (25\%). ${ }^{296}$ Compressive forces or upward displacement of abdominal contents result in diaphragmatic tears most commonly found posteriorly or centrally. The left leaf of the diaphragm is most frequently torn. ${ }^{185}$ One study reports rupture of the left leaf in $57 \%$ of cases, the right leaf in $36 \%$, and both leaves in $7 \% .{ }^{297}$
Diaphragmatic rupture is often associated with rib fractures and other thoracoabdominal injuries. These other injuries often dominate the early clinical picture. In fact, $90 \%$ of diaphragmatic tears are overlooked at the time of injury. ${ }^{298}$ The use of modern imaging techniques is expected to increase the early recognition of this injury. Rupture of the diaphragm is associated with $10 \%$ to $40 \%$ mortality. ${ }^{299,300}$ Death is often due to associated injuries. The development of complications such as abdominal abscess and pneumonia occur in approximately two thirds of cases. ${ }^{301}$

The ruptured diaphragm itself may lead to serious problems that often have a delayed onset. The size and site of the tear are the major factors in determining the development of signs and symptoms. Intermittent herniation of abdominal organs and tissues, especially on the left side, may occur.

The most serious complication of transdiaphragmatic herniation of abdominal organs is the development of tension viscerothorax, a potentially lethal lesion. The stomach may herniate into the left pleural space and develop a volvulus leading to massive gastric distention that results in compression of the lung and a shift of the mediastinum to the right side (Fig. 31.31).

Extrapulmonary airways may also be disrupted. Blunt or penetrating trauma may disrupt the trachea or bronchi. Overall, the incidence of tracheobronchial injury is $3 \%$. It carries a mortality of $30 \%$. Death is often caused by associated injuries with half of the lethally injured persons dying within 1 hour. ${ }^{291}$ Tracheobronchial disruption due to penetrating trauma usually involves the cervical trachea $(75 \%){ }^{185}$ It is often manifest by signs of upper airway obstruction. Blood with air bubbles typically flows from the wound. Tracheobronchial ruptures due to severe blunt force are relatively rare and are most commonly motor vehicle related. Several mechanisms have been proposed to explain their development: compression of the bronchus against the spine; direct compression by the sternum against a closed glottis; sudden increase in

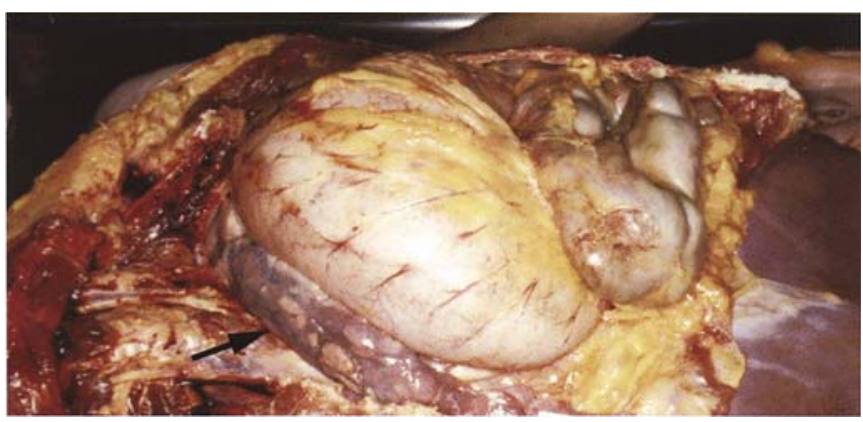

FIgure 31.31. Tension diaphragmatic hernia. The left hemithorax is filled by distended stomach and intestine. Lung (arrow) is compressed toward the midline. 
intraluminal pressure against a closed glottis; transhilar shearing forces; and deceleration of the pendulous lungs against the relatively less mobile trachea and proximal bronchi.

Ruptures predominantly involve the bronchi $(80 \%)^{294}$ and appear as tears parallel to the cartilage rings. Bronchial tears more commonly damage the right bronchus and are characteristically found within 1 inch of the carina. ${ }^{302}$ Tracheal tears are horizontal and are also found within an inch of the carina. ${ }^{294}$ Blunt force tracheobronchial ruptures are commonly accompanied by fractures of the first to third ribs.

Tracheobronchial rupture is a very serious injury often leading to pneumothorax or pneumomediastinum. Some series report $80 \%$ on-scene mortality, ${ }^{303}$ with the subsequent deaths of $30 \%$ of patients who were alive upon arrival at the hospital. ${ }^{185}$ Morbidity and mortality may also be related to obstruction of the intact airway. The airway may be obstructed by the tongue, avulsed teeth, blood, secretions, dentures, other foreign material, laryngeal or cricoid fractures, or an adjacent expanding hematoma. The onset may be acute, insidious, progressive, or recurrent.

\section{Explosions}

Several types of explosions occurring in a variety of scenarios may lead to pulmonary injury. It is an unfortunate fact of modern society that bomb explosions consequent to terrorism commonly cause death and injury. Proper interpretation of injuries and recognition, collection, and transmission of evidence not only are important in establishing the cause of death/injury but also may be critical in determining the position of the victim relative to the device and reconstructing the device and the incident. Pulmonary injury may be due to blast effect, penetration by projectiles, blunt force injuries, and thermal injuries, including inhalation of smoke/fumes (Table 31.3).

An explosion results in the formation of a high-pressure wave of compressed air (blast wave) that propagates radially from the seat of the explosion. The characteristics of the blast wave are determined by the type of explosive

TABLE 31.3. Explosion-related pulmonary injuries

Blast
Projectiles
Blunt force
Thermal
$\quad$ Primary
Secondary
Asphyxia
Smoke and fume inhalation
Compression

(high explosive-supersonic blast wave; low explosivesubsonic blast wave) and the medium through which the wave passes (air, water, solid structure). Most explosions occur on land, above ground, and thus the blast wave propagates through the air. Environmental structures may also affect the characteristics of the wave by focusing, deflecting, or reflecting it.

The incidence of fatal blast injury is variable. While uncommon in some bombing incidents, ${ }^{304}$ in other scenarios it has been reported to contribute to as high as $86 \%$ of explosion fatalities and $47 \%$ to $57 \%$ of injuries sustained by survivors. ${ }^{305}$ In general, blast injury is dictated by the size and duration of the blast wave (proportional to the size of the explosion), the proximity of the victim to the blast (wave deteriorates exponentially from the center of the explosion), orientation of the victim to the blast, environmental factors, and features of the tissues/organs. ${ }^{304,306}$

Blast wave damage is a form of barotrauma. Internal organs/tissues having air/fluid interfaces and air-filled organs are most susceptible to a blast wave traveling through the air. Damage to the organs/tissues is caused by the effects of high-frequency stress waves and lowfrequency shear waves. ${ }^{307}$ Pressure differentials at these interfaces are reinforced by reflection of the stress waves off the mediastinum and thoracic cavity. ${ }^{304,308}$

The tissue most sensitive to the effects of an air blast wave is the eardrum. ${ }^{306}$ Relatively small increases in pressure (as little as 5 psi in excess of atmospheric pressure) ${ }^{309}$ can damage the tympanic membrane. Larger pressure increases may disrupt middle ear structures. Other organs require higher pressure gradients (56 to $76 \mathrm{psi}$ ) to sustain injury. The lack of tympanic membrane rupture makes barotrauma to other organs unlikely but does not preclude it. ${ }^{306}$

Injury to the lung, the second most susceptible tissue to an air blast wave, is responsible for most deaths caused by an air blast wave. ${ }^{306}$ The extent of blast injury to the lung is a critical factor in determining mortality. The blast wave results in air-space enlargement and disruption of alveolar walls. ${ }^{310}$ Damage at the alveolar/capillary interface can lead to hemorrhage (Fig. 31.32), air embolism, pneumothorax, hemothorax, pneumomediastinum, and subcutaneous emphysema. ${ }^{311}$

Air embolism is considered a major factor in immediate deaths due to blast injury. Experimental animals dying within 30 minutes of blast injury commonly succumb as a consequence of air embolism. ${ }^{304}$ Histologic evidence of air embolism was reported by Tsokos et al. ${ }^{311}$ in four of eight blast wave human fatalities. Blast-induced hemorrhage can also play a role in morbidity and mortality. Massive hemorrhage can cause or contribute to rapid death. Delayed lethality is often due to progressive pulmonary insufficiency caused by intraalveolar hemorrhage, edema due to damage of the capillary/alveolar interface, 


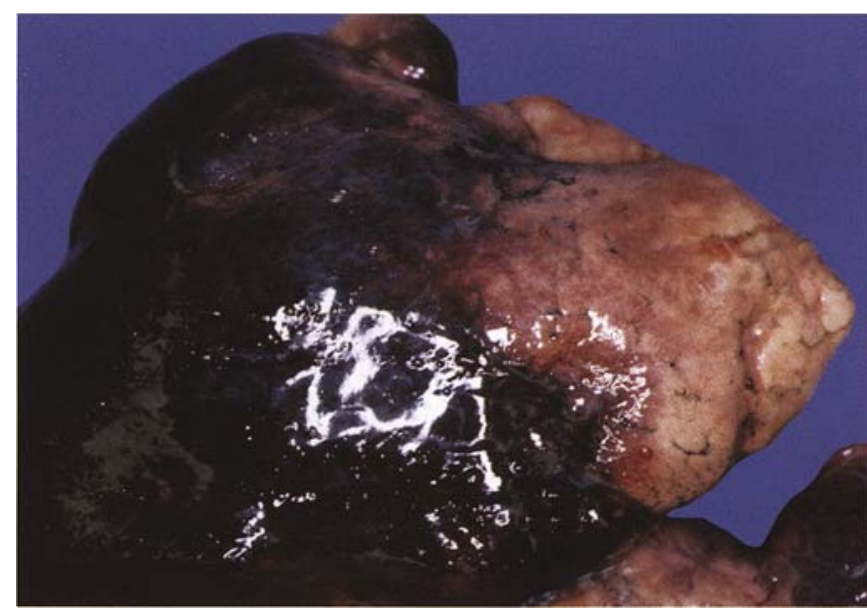

FIGURE 31.32. Lung blast injury that has caused massive pulmonary hemorrhage.

or diffuse alveolar damage. ${ }^{306,311}$ Fat embolism is also common in victims of lethal blast injury, being seen in five of the eight blast fatalities reported by Tsokos et al. and may contribute to delayed pulmonary dysfunction. Depletion of pulmonary antioxidant reserves leading to free radical oxidative stress has been postulated to further contribute to lung injury. Hemoglobin released by ruptured red blood cells has been implicated in amplification of the oxidative stress. ${ }^{312-315}$

Pulmonary death/injury can also result from penetration/perforation by projectiles (Fig. 31.33). In fact, projectiles are responsible for most deaths in bombing incidents except in incidents with massive building collapses. ${ }^{306}$ These projectiles may be components of the bomb necessary for its function (e.g., wire, timer, blasting cap), its transport or concealment (e.g., case), enhancement of antipersonnel injuries (shrapnel), or fragmented materials from disrupted articles around the device. Recovery of these materials may help ascertain the design, components, or placement of the device (Fig. 31.34) and thus help in the recognition of the sources of materials used to construct the device and the identity of the bomb

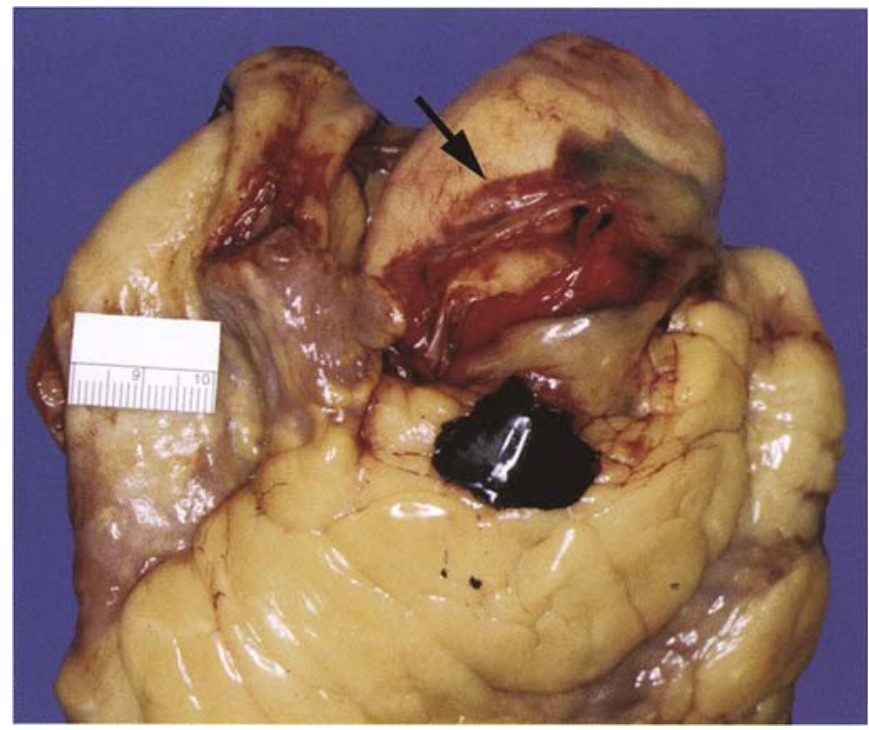

FIGURE 31.33. Explosion-related penetrating injury of the ascending aorta (arrow). A fragment of a gunpowder container is seen.

maker. Since even a single small fragment of material may contain critical information, all foreign material needs to be recovered and submitted to the legal authorities.

Explosion-related thermal effects may also lead to death/injury. These are most commonly due to fires related to structural damage with disruption of gas and electric lines. Less commonly, the heat released by the explosion may cause burns (primary burns) or ignite adjacent materials. An exception is an explosive device designed to cause injury/death via burning-incendiary device. For the most part, primary thermal burns are features of large explosions with the victim in close proximity to the seat of the explosion.

Other respiratory mechanisms of explosion-related deaths include smoke inhalation (usually structural fire secondary to the explosion), inhalation of poisonous fumes (usually in underground mine explosions),
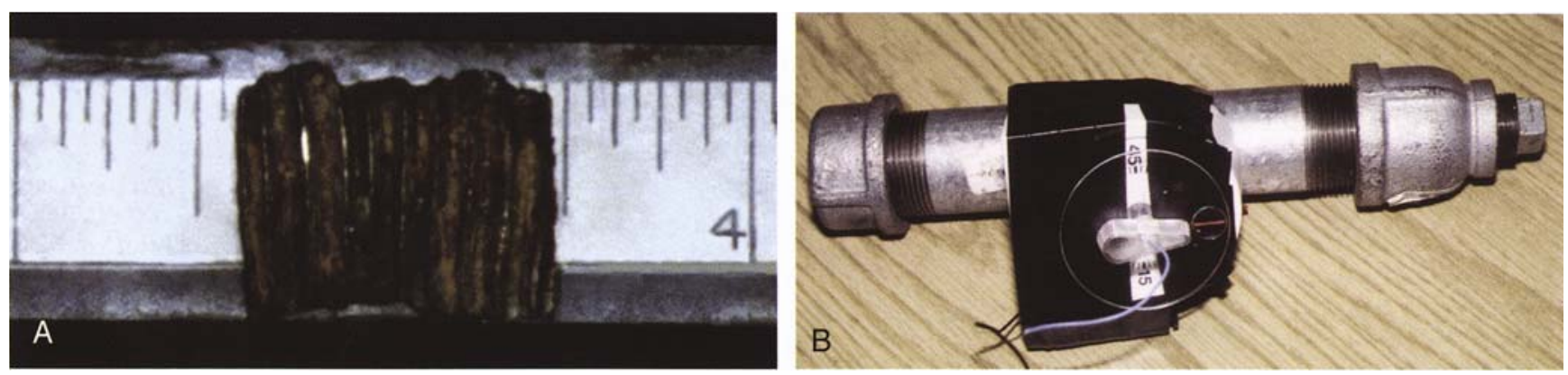

Figure 31.34. A. Threaded metal fragment recovered from lung following explosion. B. Pipe bomb (note threaded segment of pipe). 
compression asphyxia (structural collapse), and firerelated oxygen atmospheric oxygen depletion. Blunt force injuries may result from structural collapse, falls, or the victim being accelerated by the blast wave and subsequently striking a nonyielding object/structure. . $^{304,306}$

Occasionally, victims at explosion incidents may have been injured, some lethally, from causes unrelated to the explosion itself, including accomplices, security personnel, or potential witnesses eliminated by gunshots.

\section{Asphyxia and Drowning}

Asphyxia is a broad term encompassing a multi-etiologic set of conditions, mechanical and chemical, that have traditionally been thought to have as their basis some defect in the supply, uptake, or utilization of oxygen (Table 31.4). Although some of these entities have been shown to involve other pathophysiologic mechanisms, they have continued to be considered as asphyxias. These entities typically involve alteration in cerebral blood flow (e.g., neck compression by a heavy weight) or increased thoracic and cerebral vascular pressure (e.g., sudden chest compression).

There is no single finding diagnostic of asphyxia. Anatomic findings depend on several factors, most importantly the type of condition that is the cause of the asphyxia. A detailed comprehensive discussion of asphyxia is beyond the scope of this chapter. Discussion in this chapter is restricted to lower airway obstruction, aspiration, and drowning.

Obstruction to the passage of air may occur when there is sufficient swelling of tissues around or lining the airway, extrinsic compression of the airway or some intrinsic lesion obstructs the airway. Obstruction due to airway swelling usually involves the larynx, soft tissues immediately above the larynx, or the parenchymal small airways. Swelling may be induced by infections, anaphylaxis, aspiration of an irritating substance, or direct trauma to the larynx.

TABLE 31.4. Mechanical asphyxia

Strangulation
Manual
Ligature
Yoking
Hanging
Suffocation
Vitiated atmosphere
Smothering
Choking
Compression
Airway swelling
Positional asphyxia

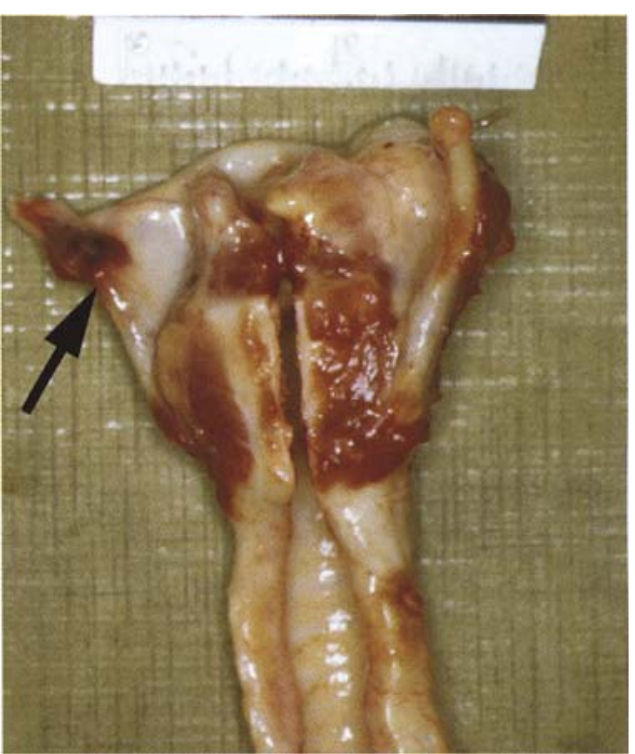

FIGURE 31.35. Larynx with fractured left superior horn (arrow).

Airway obstruction due to trauma is often the result of injury to the larynx caused by a direct blow or neck compression. Most commonly, the mucosa immediately proximal to the larynx swells as a reaction to the blow or to a fracture of the superior horns of the thyroid cartilage (Fig. 31.35). In these cases the structural integrity of the airway is maintained, and death results from progressive luminal narrowing due to the swelling. The onset of signs and symptoms of respiratory difficulty, as well as death, may be delayed for hours depending on the rate and extent of the swelling. Uncommonly, trauma may result in extensive airway fractures, typically of the lamina of the thyroid cartilage (Fig. 31.36), resulting in instability of the larynx with airflow obstruction due to collapse of the larynx during inhalation. In these cases, respiratory distress is typically evident immediately. Cervical hematomas may expand and compress the airway, usually the trachea. Mediastinal tumors, usually Hodgkin's disease, have been reported to cause sudden airway compression leading to death, often in the setting of anesthesia induction. ${ }^{316}$

Choking involves blockage of the internal airway by a foreign material. If the obstructing material is a discrete object in adults, it is generally not inhaled into the deep airway but is usually impacted deep in the posterior pharynx where it straddles the openings to the larynx and esophagus (Fig. 31.37). In contrast, choking in children often involves inhalation (aspiration) of a small object deep into the airway.

Adults who choke on an object or bolus of material are usually, although not always, impaired by such conditions as intoxication (ethanol or other depressant), neurologic 


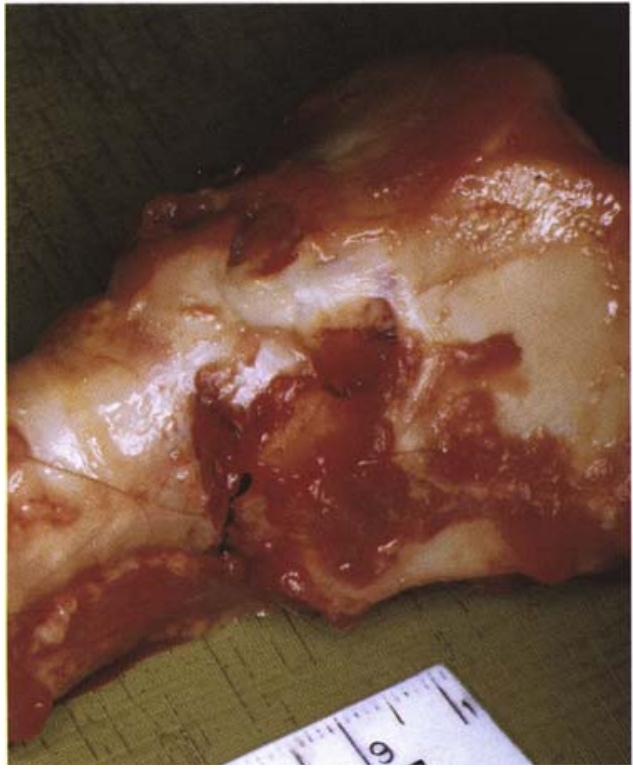

FIGURE 31.36. Larynx with fractured lamina.

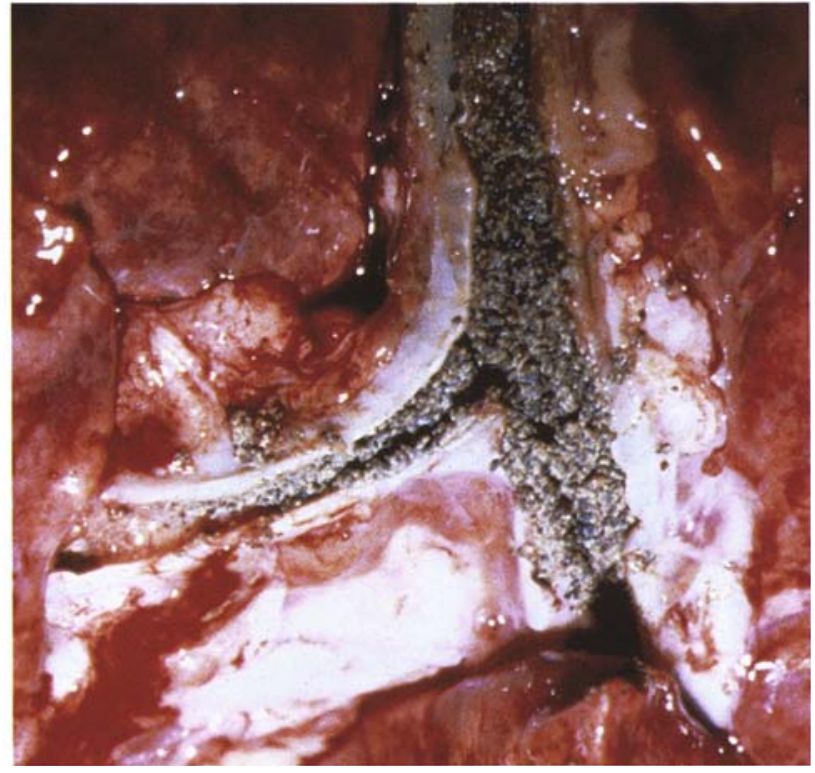

Figure 31.38. Aspiration of black pepper. Pepper is impacted in the upper airway.

The aspiration of some materials may cause obstruction by more than one mechanism and may suggest the intent behind the incident. Such a situation exists with fatal aspiration of black pepper. Aspiration of black pepper causes mechanical obstruction by its volume and mucosal edema by its irritant effect (Fig. 31.38) ${ }^{317-319}$ Piperine is the primary irritant in black pepper. ${ }^{317,319}$ The recognition of the correct mechanism or relative combination of mechanisms is important since different amounts of pepper are required for each mechanism and the time
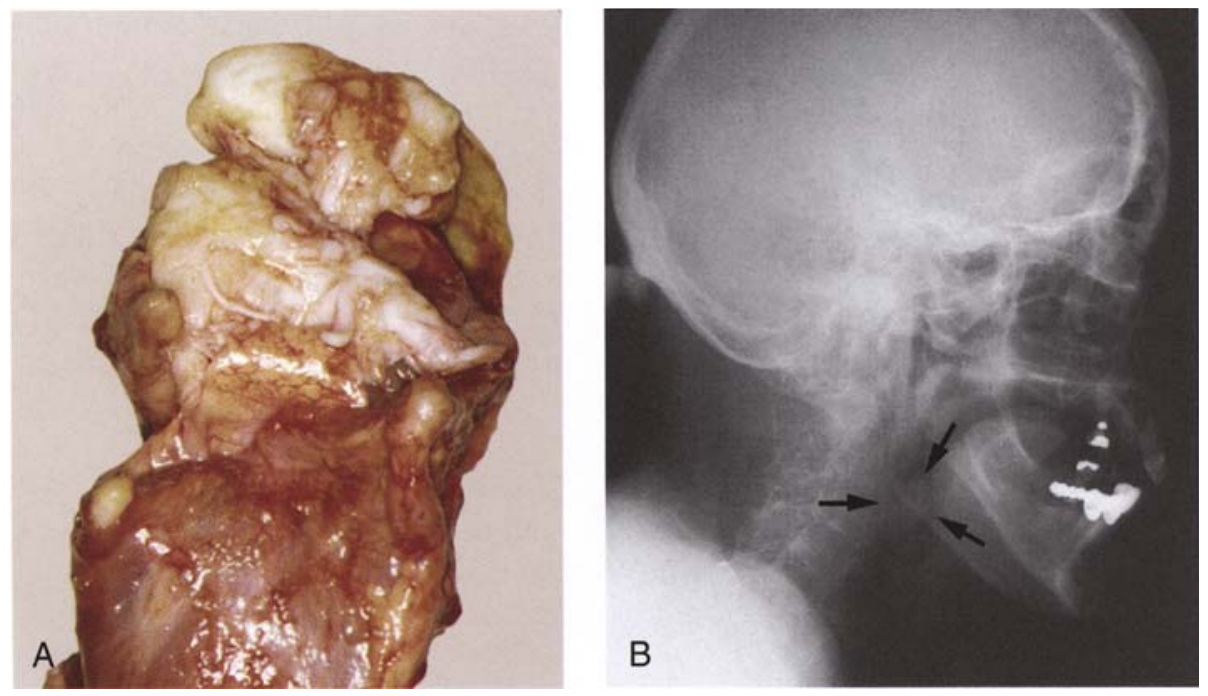

Figure 31.37. Choking. A. Bolus of boiled shrimp occludes the laryngeal orifice. B. Neck and skull radiograph depicting obstructing bolus in area of epiglottis (arrows). 
course of the fatal incident is also potentially different. It is important to attempt to quantify the amount of pepper (dry weight or volume) within the airways and elsewhere. The presence of preexisting conditions such as reactive airway disease can also influence the outcome of pepper inhalation. In a series of eight lethal pepper aspiration cases reported by Cohle et al., ${ }^{317}$ seven were the result of child abuse. The victims are characteristically young children who are administered pepper as punishment by their caregivers. ${ }^{317,320,321}$ The death of the other child in the Cohle et al. series was accidental and occurred in a child with documented pica, as was the child reported by Sheahan et al. ${ }^{318}$

\section{Gastric Aspiration}

Aspiration of other nondiscrete materials also occurs. Perhaps the most common of these is aspiration of gastric contents, which, although frequently observed in decedents, is an unusual cause of death. Aspiration of gastric contents most commonly occurs as an agonal or postmortem event in individuals dying of unrelated conditions (Fig. 31.39). Agonal gastric aspiration may hinder resuscitation efforts and may lead to severe respiratory problems in surviving patients. When gastric aspiration occurs as an antemortem clinically significant event, it is usually in the setting of impaired consciousness with inadequate airway protection or an ineffective swallowing mechanism. The administration of anesthesia is a major risk for

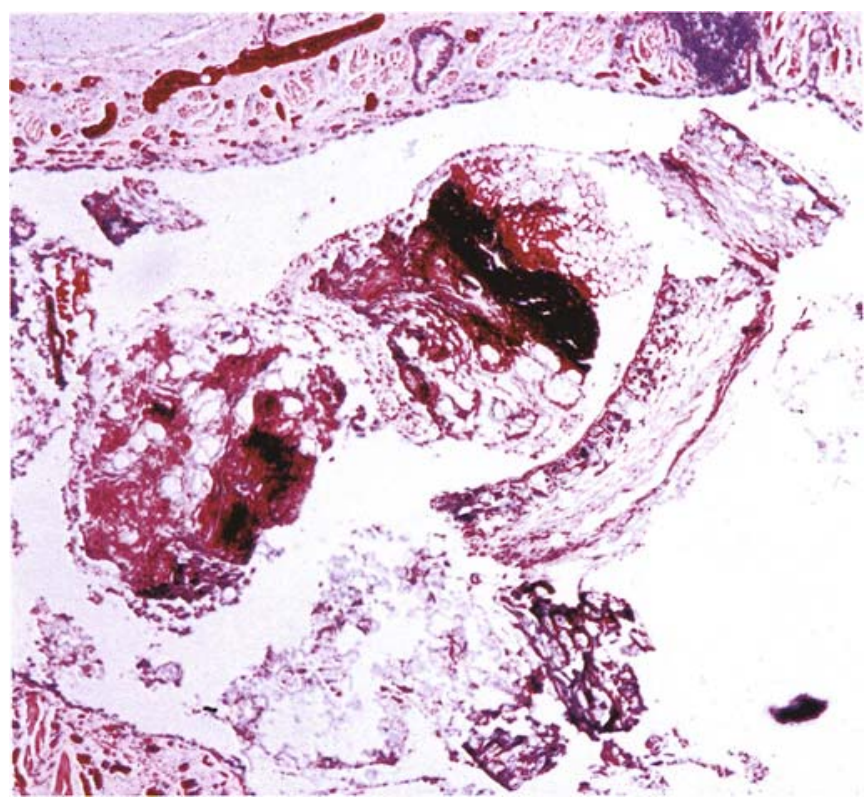

Figure 31.39. Agonal aspiration of gastric content that lies loosely in the airway (cause of death: gunshot wound of head).

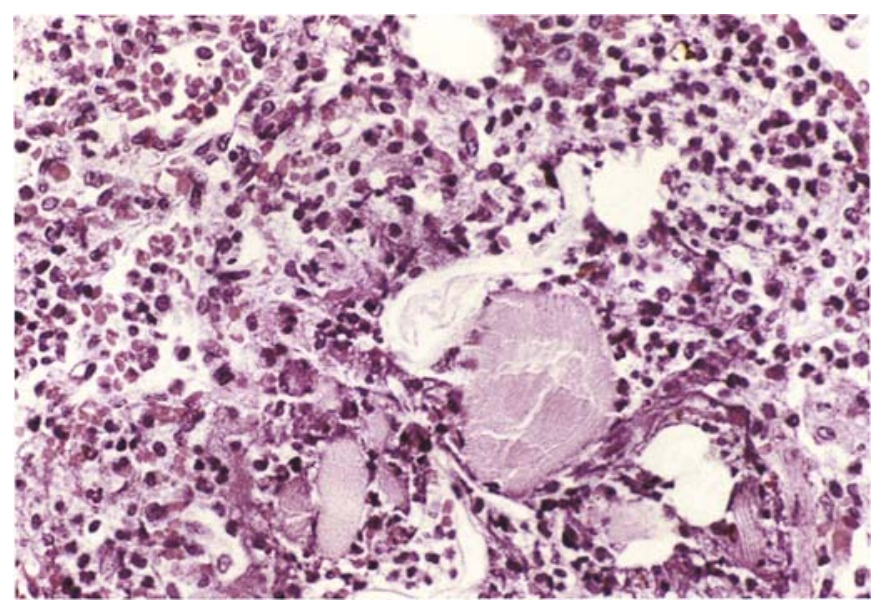

FIGURE 31.40. Aspiration pneumonia. Note fragment of food material in center of acute inflammatory exudate.

developing aspiration, especially in emergency situations where anesthesia is being administered to a patient with a full stomach.

The respiratory injury that results from aspiration depends on the acidity of the material, its amount, the presence and characteristics of any particulate material, and the introduction of bacteria. ${ }^{322}$ The aspiration of acidic gastric material $(\mathrm{pH}<2.5)$ is associated with immediate damage to the lung parenchyma resulting in diffuse alveolar damage. It is has been estimated that at least $0.4 \mathrm{~mL} / \mathrm{kg}$ must be aspirated to cause significant lung injury. ${ }^{323}$ The effects of aspirating gastric material having a $\mathrm{pH}$ in excess of 2.5 will depend on the volume, tonicity, and the presence and characteristics of particulate material. ${ }^{322}$ Aspiration of clear non-acidic liquid such as water results in hemorrhage, pulmonary edema, separation of endothelial cells from basement membranes, and peribronchial acute inflammation. ${ }^{324-326}$ Little necrosis of alveolar cells takes place. ${ }^{326}$ If the aspirated material contains large particles, obstruction of large airways occurs and death may rapidly ensue from asphyxia. ${ }^{322}$ Aspiration of material containing small particles results in extensive, prolonged pneumonia. Early in its course the inflammatory infiltrate contains neutrophils (Fig. 31.40) and has a foreign body or granulomatous component (see Fig. 5.7 in Chapter 5). Later, the infiltrate is mononuclear. Foreign body granulomas persist. ${ }^{322}$

Infection accompanying aspiration falls into two general scenarios. Aspiration of a small heavily contaminated inoculum results in necrotizing bronchopneumonia, often with abscess formation (Fig. 31.41). Empyema may also develop. These infections are usually caused by oropharyngeal bacterial flora, typically anaerobic organisms. ${ }^{322}$ The development of these infections is affected by the person's dental hygiene, overall health status, and 


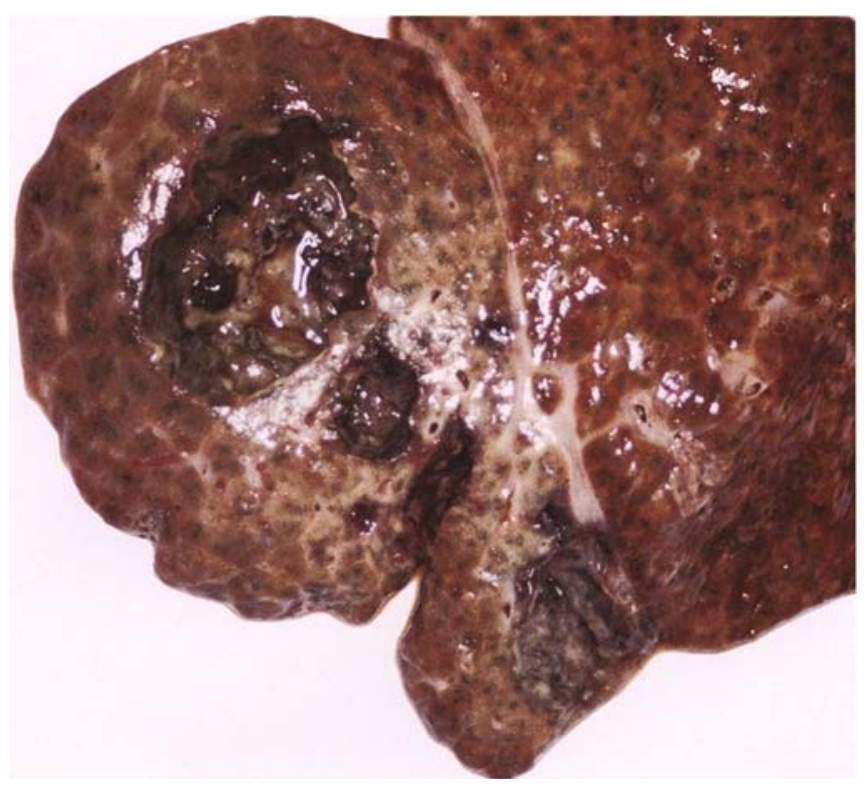

FIGURE 31.41. Lung abscess as a complication of aspiration of orogastric material.

the effectiveness of the cough reflex and other pulmonary defense mechanisms (see Chapter 5). ${ }^{322}$ Some patients who have sustained damage from large aspiration episodes, primarily of the acid type, develop superimposed bacterial pneumonia. Aerobic bacteria are typically the responsible agents. ${ }^{322}$

Not all aspirated material is foreign to the body, although it may be foreign to the airway. Aspiration of blood from injuries or diseases stemming from the head or neck may block the airway. Similarly, hemorrhage from pulmonary lesions such as tumors, cavitary tuberculosis, and other necrotizing inflammatory processes may erode vessels of substantial size and result in either airway obstruction or, in some cases, exsanguination.

\section{Drowning}

Drowning has traditionally been considered as death due to respiratory impairment from immersion in a liquid, usually water. The definition of drowning was updated at the 2002 World Congress on Drowning:

\footnotetext{
"Drowning" is a process resulting in primary respiratory impairment from submersion/immersion in a liquid medium. Implicit in this definition is that a liquid/air interface is present at the entrance of the victim's airway, preventing the victim from breathing air. The victim may live (with or without sequelae) or die after this process, but whatever the outcome, he or she has been involved in a drowning incident. ${ }^{327}$
}

In most cases the person is submerged in the water. However, total submersion is not required and drowning can result when only part of the body is in the liquid as may occur, for example, to a person face-down in shallow water. Approximately 9000 persons drown annually in the United States, ${ }^{328}$ making it second to motor vehicle accidents as the most common cause of accidental death and in some areas it is the most common cause of death among children. ${ }^{329-331}$

Although considerable variations exist, most drowning victims and drowning episodes fit within a limited number of well-recognized scenarios. Most drowning victims are male and are most likely to be either toddlers or teenagers. ${ }^{332,333}$ Healthy adults who can swim rarely drown unless there is an intervening factor making them less likely to survive in the water, such as natural disease, alcohol/ drugs, injury, or engaging in activity beyond their capabilities or in a dangerous environment.

Not unexpectedly, detailed observations of humans undergoing the drowning process are lacking. Much of our understanding of the drowning process comes from animal studies carried out many years ago. Based on these animal studies, drowning has been divided into five steps. $^{334}$

Drowning is characterized initially, especially in cold water, by a period of panic and hyperventilation. Breathholding may be attempted and is usually followed by a period of involuntary laryngospasm due to the presence of liquid in the oropharynx or larynx. ${ }^{335}$ The victim develops hypoxia, hypercarbia, and acidosis ${ }^{336}$ and, at some point, laryngospasm abates. ${ }^{337}$ Uncontrollable hyperventilation may lead to aspiration of variable, sometimes large, amounts of water that may increase the risk of vomiting and aspiration of gastric contents. Many drowning victims exhibit violent struggling prior to losing consciousness. ${ }^{335}$ Once unconsciousness occurs, water can passively enter the airway.

Noble and Sharpe ${ }^{338}$ have attempted to reconstruct the events that occur for human drowning victims; however, this reconstruction is not from direct observation but rather extrapolated from animal experiments. This reconstruction is reasonable for a human who suddenly is submerged while conscious and cannot break the surface of the water. However, Modell et al. ${ }^{334}$ have reported that this sequence of events is rarely observed by witnesses to a drowning. In some cases, such as in shallow water blackout due to presubmersion hyperventilation, unconsciousness precedes the onset of the drowning process and breathing underwater occurs without evidence of panic or struggle. In most cases of human drowning the body of the drowning victim is found either underwater or floating on the surface without an eyewitness to the immediately preceding events. ${ }^{334}$

The major pathophysiologic consequence of drowning is prolonged hypoxia with resultant metabolic acidosis. ${ }^{339,340}$ The hypoxia is due predominately to the aspiration of water. However, the amount of liquid inhaled varies considerably from individual to individual. ${ }^{337}$ In 
humans, aspiration of 1 to $3 \mathrm{~mL} / \mathrm{kg}$ has been associated with profound alterations in pulmonary gas exchange and decreased pulmonary compliance. ${ }^{341-344}$ In most cases of drowning it is possible to demonstrate the presence of the characteristic foamy/frothy fluid seen after water aspiration, ${ }^{334}$ a condition often referred to as "wet" drowning.

It has often been written that no significant water aspiration occurs in $10 \%$ to $15 \%$ of cases $, 334,335$ so-called "dry" drowning. This estimate is primarily based on animal studies reported in the 1930s and 1940s. ${ }^{345-347}$ Theories advanced to explain these cases include laryngospasm, vagus-mediated cardiac rhythm disturbances, pulmonary reflex, and absorption of fresh water into the systemic circulation. ${ }^{345,348-350}$

However, establishing the presence of dry drowning is problematic. ${ }^{334,351}$ No specific diagnostic criteria are available, different studies are based on ill-defined or unstated criteria, and, in some cases, the diagnosis appears based on the weight of the lungs. Reviews of the experimental data and human case reviews have led to either questioning or discounting the concept of dry drowning. Review of the original animal experiments of $\operatorname{Cot}^{346}$ indicates that all animals subjected to the complete drowning process demonstrated the foamy/frothy fluid characteristic of water aspiration. ${ }^{334}$ In some cases Cot could not retrieve water from the lungs, a situation that suggested the lack of aspiration. However, in other studies using water with some visible marker as the drowning medium, all animals demonstrated diffuse distribution of the water throughout the lungs. Other authors have observed that in cases of fresh water drowning in dogs in which no water could be drained from the lungs, there was evidence of strong hemodilution attributed to rapid admittance of water from the lungs into the blood with resultant ventricular fibrillation. ${ }^{334}$ Some authors consider dry drowning as either cases of natural death occurring in the water or disposal of dead bodies in water. ${ }^{334,352}$

The author agrees with the conclusions reached at the 2002 World Congress on Drowning that "all drownings are wet" and that the terms wet drowning and dry drowning should be abandoned. ${ }^{327}$

Drowning can occur in all types of water-fresh, salt, or brackish. When fresh water is aspirated, the water rapidly moves from the air spaces into the blood vessels, removes surfactant, and damages the pneumocytes. ${ }^{335} \mathrm{In}$ contrast, when salt water is inhaled, fluid moves from the blood vessels into the lung tissue and air spaces, leading to the rapid onset of protein-rich pulmonary edema. Salt water also leads to the destruction of surfactant and capillary damage. ${ }^{353}$ The loss of surfactant leads to decreased compliance and atelectasis and marked ventilation-perfusion mismatch. ${ }^{335}$

Although movement of water into or out of the circulation (fresh versus salt water) conceivably could alter the blood's electrolyte concentrations, in most cases this does not seem to be a major issue from a clinical standpoint except in water having a high electrolyte content, such as the Dead Sea. ${ }^{335,354}$ However, the role, if any, of electrolyte changes in persons dead in the water is not known. It is generally agreed that the amount of water aspirated is more significant than the type of water. ${ }^{337,355,356}$

The temperature of the water can also have an effect on the drowning process, especially when the water is cold. Cold water, especially $10^{\circ} \mathrm{C}$ or colder, has significant cardiovascular effects and may also trigger a gasp reflex with subsequent hyperventilation. ${ }^{357}$

Aspiration of particulate, chemical, or biologic contaminants in the water may further compromise the lungs, as may the aspiration of gastric contents. Some individuals who survive the initial submersion incident develop pneumonia or diffuse alveolar damage hours to days later.

The investigation of the circumstances and the scene of submersion are paramount in properly establishing not only the cause of death but also the manner of death since the autopsy findings of a drowning victim may well be identical whether the victim jumped, fell, or was pushed into the water. Since humans by nature are not aquatic animals, human excursions into water may be viewed as humans versus a hostile environment. Investigating drowning involves determining what human and environmental factors prevented the victim from leaving the water alive and healthy. In addition, the fact that a body was found in water does not necessarily indicate that death was due to drowning or that, in fact, the person was alive when he or she entered the water.

There is no diagnostic anatomic finding conclusive of drowning. Most of the findings at autopsy are due to the body being in water, regardless of whether or not death was caused by drowning. In addition, the typical postmortem changes that occur may be markedly altered due to water immersion. Petechiae on the surface of the body can occur in drowning but are uncommon, nonspecific, and, when present, are usually not very plentiful. Pleural and epicardial petechiae are not unusual, but are also nonspecific. The finding that perhaps is the most suggestive indication that a person was alive while in the water is the presence of foam emanating from the nose or mouth (Fig. 31.42). ${ }^{334}$ The foam is caused by admixing moving air with water. The foam is often white, off-white, or blood-tinged. The foam cone is often quite prominent in drowning victims and will often re-form when wiped away. However, foam emanating from the nasal and oral apertures is not always present in drownings. Lunetta et al. ${ }^{358}$ reported its presence in $17 \%$ of bodies recovered from water. Froth is common within the trachea and more distal portions of the airways.

It should be recognized that the pulmonary foam/froth is not specific for drowning or breathing while submerged and may be seen in other conditions with copious pulmo- 
nary edema during agonal breathing (e.g., heroin intoxication). However, in the absence of other potential causes, the presence of a foam cone is a strong indication that the victim was breathing while in the water. The absence of foam does not necessarily indicate that the person was dead when submerged. However, the author agrees with Modell et al. ${ }^{334}$ that "one is on shaky ground by making a diagnosis of drowning at autopsy in the absence of the characteristic foamy/frothy fluid seen after water aspiration."

Other more nonspecific findings may be found in the lungs. The lungs of drowning victims are usually heavy because of the presence of pulmonary edema and liquid aspirated from the drowning medium. The lungs may appear hyperexpanded ${ }^{358,359}$ (they often bulge from the open chest cavity during autopsy) due to the presence of water and entrapped air (Fig. 31.43). Pleural effusion is also a common finding in drownings, being reported in $53 \%$ of cases occurring in individuals at least 16 years of age. ${ }^{360}$

The presence of water, gastric contents, plant material, or sand/silt in the mouth, airways, lung, or stomach is of no diagnostic significance since these materials can passively reach these locations after death. The presence of water in the sinuses is also not useful diagnostically in determining drowning since water can also reach these areas passively while the body is submerged. The presence of congestion or hemorrhage in the mastoid air cells is also not specific for drowning.

There are no microscopic features that are diagnostic of drowning. Common findings include alveolar distention, alveolar septal thinning and disruption, capillary congestion, air-space edema fluid/hemorrhage, and, on occasion, exogenous particulate material in the airways. ${ }^{344}$

The diagnosis of drowning is further complicated by the fact that there are no specific laboratory tests to prove

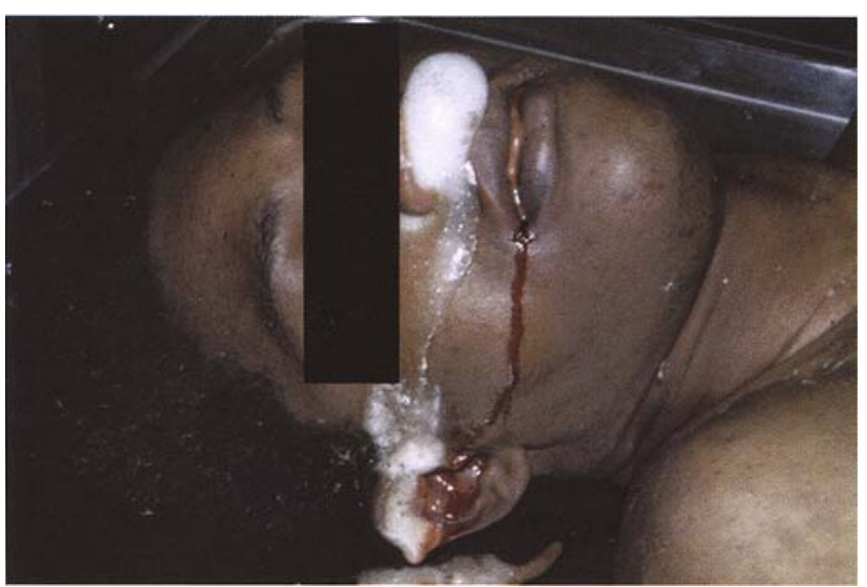

Figure 31.42. Drowning. Foam cone.

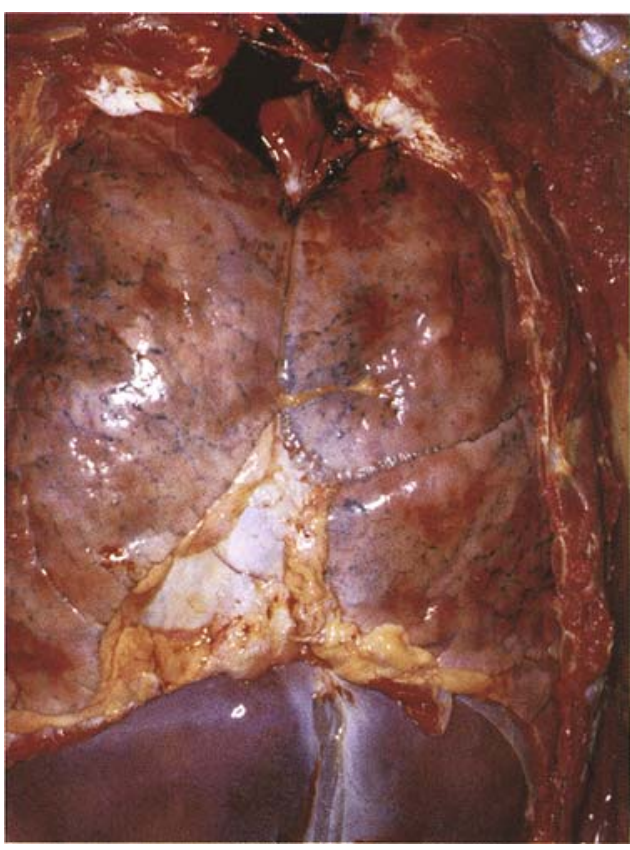

Figure 31.43. Drowning. Hyperexpanded lungs.

drowning. ${ }^{334}$ Some studies have been performed to assess the absorption and circulation of diatoms found within the water aspirated during drowning. At this time, these studies have not been fully validated, ${ }^{334,344,361,362}$ require special expertise, and are not generally available. Other so-called drowning tests have been proposed from time to time and have proven unreliable. ${ }^{334}$

Drowning can be difficult to diagnose. However, when appropriate investigative information about the victim, circumstances, and scene are coupled with the findings of the postmortem examination, the correct diagnosis can usually be made.

\section{Dysbarism}

Dysbarism encompasses a variety of pathologic changes caused by the effects of altered environmental pressure on closed air spaces (barotrauma) or problems caused by breathing gases at elevated partial pressures (decompression illness). Most changes are acute but occasionally may be delayed for months or years (e.g., dysbaric osteonecrosis $^{363,364}$ and dysbaric retinopathy ${ }^{365,366}$ ). Most commonly seen in scuba divers ("scuba" is an acronym for self-contained underwater breathing apparatus) and compressed-air workers (e.g., tunnel workers laboring under increased atmospheric pressure), dysbarism is occasionally seen in situations where there is a sudden reduction in pressure at conditions of high altitude. ${ }^{367}$

In 2001, there were 9 million certified scuba divers in the United States. ${ }^{368}$ During 1980 to 1996,1290 deaths 
were associated with scuba activities. Drowning is the most common cause of death during diving and reflects a number of factors including inexperience, entrapment, entanglement, injury, inadequate air supply, and natural disease. Among divers over the age of 35 years, cardiovascular disease is the second most common cause of death during diving. ${ }^{369}$ In a series of 60 diving fatalities during 1996 in which autopsy reports were available for review, decompression illness caused or contributed to 11 of the deaths-10 due to arterial gas embolism (AGE) and one to decompression sickness. ${ }^{369}$ Although decompression sickness is an uncommon cause of scuba-related death, it is an important contributor to morbidity. ${ }^{369}$

During descent while diving, the volume of gas decreases as it is compressed by the pressure of the water. Normally, gas pressure in the various air-filled spaces of the body and equipment is in equilibrium with the environment. ${ }^{367}$ If the passages for gas exchange are blocked and a change in ambient pressure occurs, pressure disequilibria occur. Discomfort due to pressure disequilibria during descent mainly affect the ears, face (a face mask's entrapped air), sinuses, and lungs. ${ }^{367,368}$ The latter, known as lung squeeze, is often manifest by pulmonary edema and frothy hemoptysis. ${ }^{367}$

Serious pulmonary dysbarism is most often related to ascent ${ }^{359}$ and reflects the expansion of gas volume as the ambient pressure decreases toward the surface of the water. ${ }^{367}$ Decompression illness encompasses two entities-pulmonary barotrauma of ascent (pulmonary overpressurization syndrome [POPS]) and the decompression syndrome. . $^{367,369}$

Overdistention and resultant tearing of alveolar septa by entrapped expanding gas allow the gas to reach extraalveolar sites (Fig. 31.44). Pulmonary overpressurization syndrome most commonly is associated with rapid uncon-

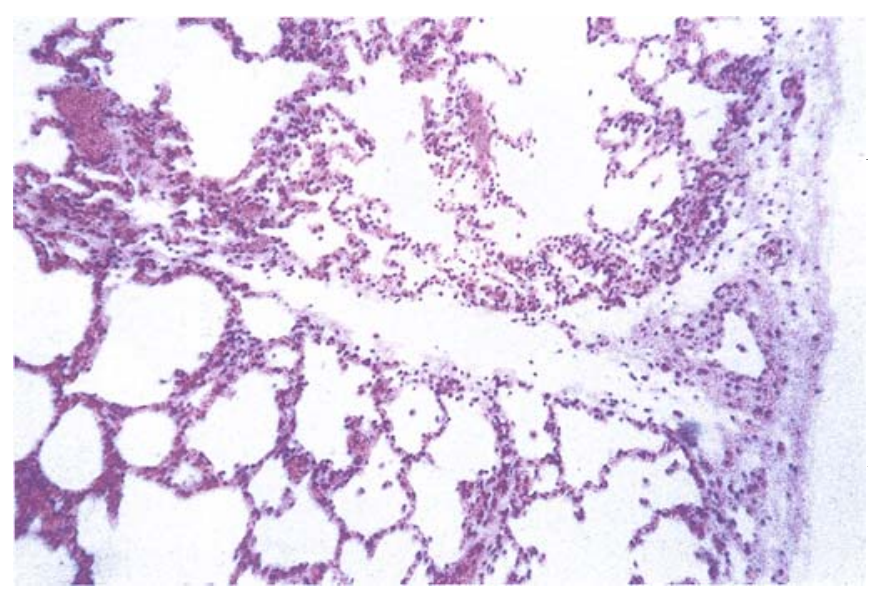

FIGURE 31.44. Extraalveolar syndrome. Interlobular septum is widened by dissecting air. trolled ascent and may occur during ascent from as shallow as 6 feet, especially if the diver is breath holding. Other factors favoring air entrapment include bronchospasm/reactive airway disease, structural lesions impeding egress of air (bullae, endobronchial mass lesions), and too-rapid ascent. ${ }^{367}$

The most serious and feared manifestation of POPS is AGE. Other manifestations include pneumothorax, pneumomediastinum, subcutaneous emphysema, alveolar hemorrhage, and venous gas embolism. Signs and symptoms of POPS occur almost immediately upon surfacing, an important point to remember when evaluating scuba deaths. ${ }^{367,370}$

The other main category of decompression illness is the decompression syndrome. It is a multisystem disorder caused by rapid decrease in ambient atmospheric pressure causing inert gas (usually nitrogen) to come out of solution and form bubbles in tissues and venous blood.

The clinical manifestations of decompression syndrome are often categorized as follows: type I, which entails cutaneous, lymphatic, or musculoskeletal manifestations; and type II, which entails focal neurologic or cardiopulmonary signs and symptoms. ${ }^{367}$ Pulmonary manifestations ("chokes") are those of venous gas embolism. Patients may have cough, dyspnea, chest pain, cyanosis, and pulmonary edema. ${ }^{371}$

The onset of signs and symptoms usually occurs within 1 hour of surfacing. Most other cases become manifest within 6 hours. Infrequently, the onset may be delayed 24 to 48 hours. ${ }^{367}$ Also, high-altitude flying may precipitate signs and symptoms of the decompression syndrome, such as may occur in a commercial aircraft even though the cabin is pressurized. Treatment of all forms of decompression illness is recompression and supportive. ${ }^{372}$

The evaluation of a death associated with compressed gas diving requires, in addition to the usual components, investigation of the person's dive history (past and current) and equipment. Consultation with someone knowledgeable about diving and dive physiology/ medicine may be very helpful in the correct resolution of these cases. A protocol for evaluating recreational scuba diving fatalities is available from Divers Alert Network (DAN). ${ }^{369}$

\section{Smoke Inhalation}

There are a wide variety of toxicants capable of damaging respiratory structures when inhaled. In general, these agents cause airway epithelial necrosis or pulmonary parenchymal damage often leading to pulmonary edema or diffuse alveolar damage. One of the most common pulmonary toxicants is smoke.

Approximately 10,000 persons die annually in the United States due to fires. The vast majority of the deaths 
is due to the effects of thermal burns and smoke inhalation. At least half of the deaths are attributable to the effects of smoke inhalation, the primary cause of death in structural fires. Patients hospitalized with smoke inhalation have an overall mortality of $5 \%$ to $8 \% \cdot{ }^{373}$ However, the overall mortality due to smoke inhalation is higher since many victims of lethal smoke inhalation do not reach the hospital alive. If the smoke inhalation is accompanied by major burns, the mortality is greater than that of either one alone. The inhalation of hot gases can also play a major role damaging the airway. Morbidity and mortality associated with structural fires strongly reflect the effects of smoke inhalation.

Smoke is a complex mixture of water vapor, other combustion gases and chemicals (carbon monoxide, carbon dioxide, cyanide, acids, aldehydes, oxidants), and particulates. The exact composition of smoke depends on the fuel sources, amount of heat generated by the fire, and the completeness of combustion. The irritants, asphyxiants, and systemic toxicants found in smoke can all have deleterious effects on a person. Factors affecting injury due to inhalation include particle size, water solubility of chemicals in the smoke (either as gases or associated with particulates), and the heat capacity of inspired gases.

The inhalation of heated air is capable of causing significant damage to the airway. Pure hot air inhalation injury is occasionally the only respiratory tract damage seen in some fires, notably flash fires. Airway injury due to heated air is generally confined to the supraglottic tissues due to the rapid cooling it undergoes as it flows down the airway. An exception to this is the inhalation of steam. The heat carrying capacity of steam is 4000 times greater than that of hot air. ${ }^{374}$ Steam is capable of maintaining its heat as it passes down the airway and is capable of causing bronchial damage. Signs of potential inhalation injury include singed facial or nasal hair, carbonaceous sputum, facial burns, and extensive burns, in general. The manifestations of inhalation injury may be delayed for up to 24 hours.

As a general irritant, smoke causes bronchorrhea, bronchoconstriction, and edema of the airway mucosa. It may contain toxic gases of variable water solubility that cause epithelial damage and necrosis on contact. Some of these toxic chemicals are attached to particulates and exert their effect when the particulates come to rest along the airway. Water-soluble chemicals and large particulates predominately affect the proximal airway, whereas insoluble chemicals and small particulates can travel to the distal portions of the respiratory tract and predominately affect the lung parenchyma.

The mechanisms by which smoke damages the lung parenchyma are varied and complex. Recent work has implicated nitric oxide (NO) (loss of pulmonary vasoconstriction leading to ventilation-perfusion mismatch), peroxynitrite (tissue injury), poly(adenosine diphosphate
[ADP]-ribose)-polymerase, and airway obstruction in causing pulmonary parenchymal damage. ${ }^{375}$ The development of smoke-induced diffuse alveolar damage is associated with enhanced mortality. A further increase in mortality occurs if bacterial pneumonia is superimposed on the smoke-related damage.

Cutaneous burns can affect the respiratory system. Patients with greater than $30 \%$ body surface area burns are susceptible to the development of capillary increased permeability at sites remote from the burned areas. ${ }^{376,377}$ This can lead to the development of pulmonary edema. The development of the increased permeability is markedly increased if there is also smoke inhalation. ${ }^{378}$

Asphyxia may also have a role, often the primary role, in causing fire-related morbidity and mortality. Asphyxia may be due to a fire-induced vitiated atmosphere. Air normally has an oxygen content of approximately $21 \%$. Consumption of oxygen during combustion may lower the oxygen content to as little as $10 \%$. Breathing oxygendeficient air may lead to rapid unconsciousness and can eventuate in death.

The asphyxiant gas that is the primary toxicant during a fire is carbon monoxide (CO), which avidly binds to hemoglobin (240 times stronger than hemoglobin). It not only causes decreased oxygen carrying capacity but also shifts the oxygen dissociation curve to inhibit release of oxygen. In addition, it acts as a cellular poison by its binding to the cytochrome system. Individuals dying of $\mathrm{CO}$ toxicity, even if no fire is involved, often demonstrate widespread loosening and slippage of epidermal (Fig. 31.45) and mucosal surfaces. These areas of epidermal slippage are difficult, if not impossible, to distinguish from partial thickness burns. Often the pattern of the slippage and lack of associated burning of hair and clothing facilitates correct attribution to $\mathrm{CO}$. Heat generated by the fire may exacerbate the epidermal slippage.

Other toxic gases such as cyanide $(\mathrm{CN})$ produced during a fire may contribute to death or debility. These other gases are often produced during the burning of plastics or other synthetic materials. Significant elevations in $\mathrm{CN}$ derived from smoke inhalation rarely occur in the absence of elevations of blood CO.

One of the most important issues that arise in relation to persons who are dead at the fire scene is whether or not they were alive during the fire. The demonstration of active uptake of smoke or gases produced during the fire reliably establishes breathing, and therefore life, during the fire.

Carbon monoxide, a product of incomplete combustion of carbonaceous materials, is prominent in most structural fires. The environment of some fires may contain up to $10 \% \mathrm{CO}$. Breathing smoke from a structural fire usually results in a rapid increase in carbon monoxide hemoglobin ( $\mathrm{HbCO}$ ) saturation. The rate of elevation is dependent on the concentration of $\mathrm{CO}$ in the 

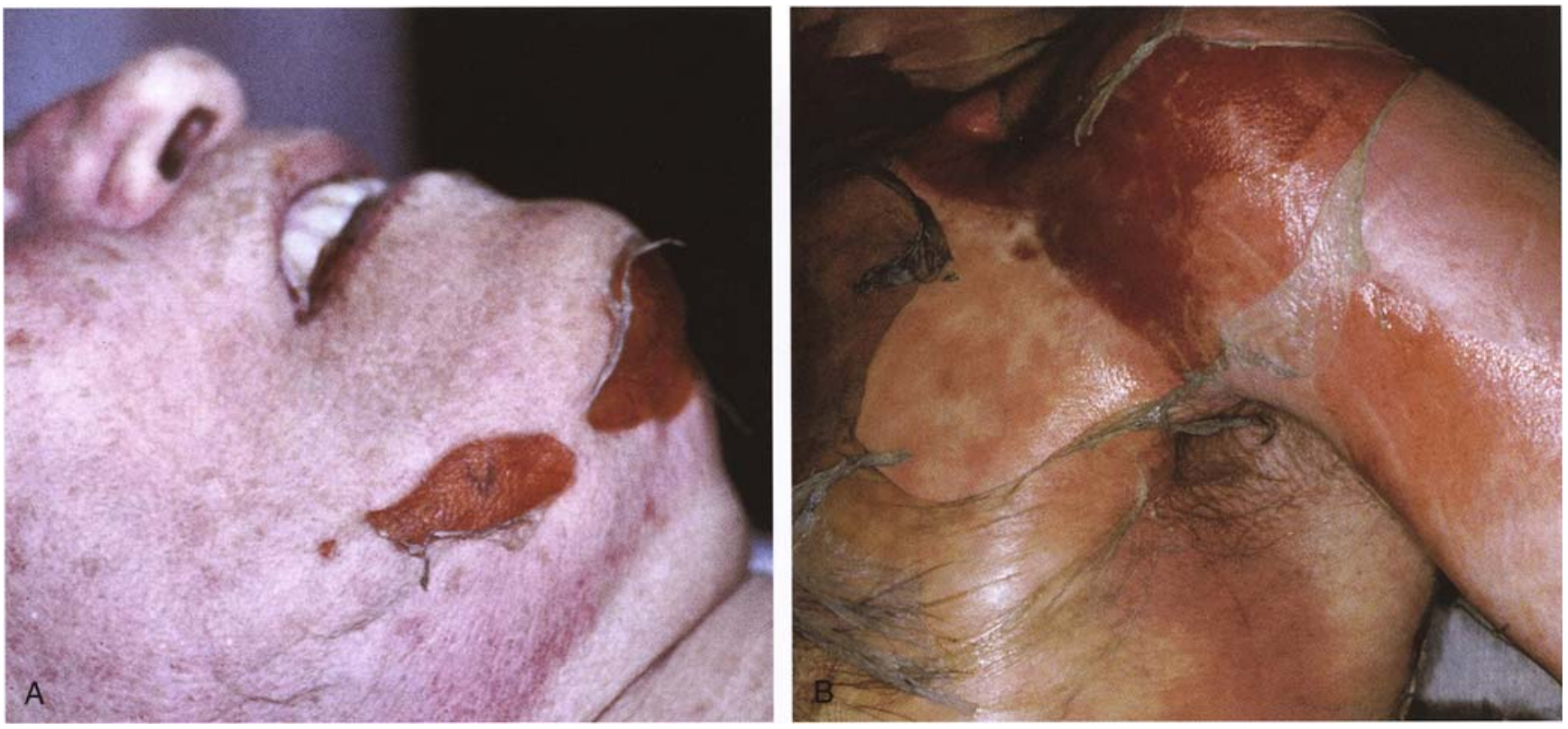

Figure 31.45. Carbon monoxide intoxication. A,B. Epidermal slippage resembling burns.

smoke. Carbon monoxide is not absorbed passively after death. Elevated concentrations of $\mathrm{HbCO}$ are indicative of breathing in the presence of $\mathrm{CO}$. Most fire victims dying of smoke inhalation will have $\mathrm{HBCO}$ concentrations in excess of $40 \%$. However, this is not always the case. Debilitated persons or those suffering from ischemic heart disease may succumb when $\mathrm{CO}$ concentrations are in the range of $25 \%$.

Isolated elevation of $\mathrm{CO}$ is not absolute proof that the victim was alive during the fire. Not all $\mathrm{HbCO}$ is necessarily derived from smoke from structural fires. The inhalation of smoke or exhaust from other sources can also elevate $\mathrm{HbCO}$. People exposed to tobacco smoke often show mild degrees of $\mathrm{HbCO}$ elevation, usually less than $12 \%$ and almost always less than $20 \%$. A person could have a lethal elevation of $\mathrm{CO}$ from some other source, such as the exhaust from an old car (prior to efficient catalytic converters) in a closed garage with the alreadydead body exposed to fire when the garage ignites.

Breathing during the fire is established by demonstrating soot in the lower airway. Soot can reach the nasal cavity, oral cavity, and throat passively during a fire in the absence of breathing. However, an intact airway with soot distal to the vocal cords is indicative of breathing during the fire (Fig. 31.46). Some fires produce minimal smoke that is not apparent during examination of the airways. Infrequently, a person reliably witnessed to be alive in a residential fire with copious smoke production will fail to have demonstrable soot in the airway. Some of these cases may be due to the rapid onset of laryngospasm; however, the author has seen rare cases in which the victim was heard screaming while in the smoky house, yet no soot was seen in the airway (and no $\mathrm{CO}$ was demonstrable in the blood).

The best assessment of breathing during the fire is made by combining the examination for $\mathrm{HbCO}$ with the examination for airway soot. The presence of soot in the distal airway and a significantly elevated $\mathrm{HbCO}$ is a reliable indication of recent smoke inhalation.

Although the presence of significant elevation of $\mathrm{HbCO}$ or soot in the distal airway establishes that the victim was alive during the fire, the lack of one or both of these findings does not definitively establish death prior to the fire. Some fires, such as flash fires, explosions, or fires in open areas may not produce large amounts or high concentrations of $\mathrm{CO}$ or smoke.

The demonstration that someone was alive during the fire does not necessarily indicate that he or she died as a result of the fire. Occasionally, a victim dying of a non-fire-related injury (e.g., gunshot wound of head) will die during the early stages of a concealment fire after some nonlethal amount of smoke has been inhaled. Similarly, some victims with potentially lethal nonfire-related injuries will be killed by the fire. Being alive during the fire does not equate with being conscious or nondebilitated.

As with almost all types of death, careful evaluation of the history, scene, and body (including toxicologic studies) will give the pathologist the best opportunity to correctly assess the death. 

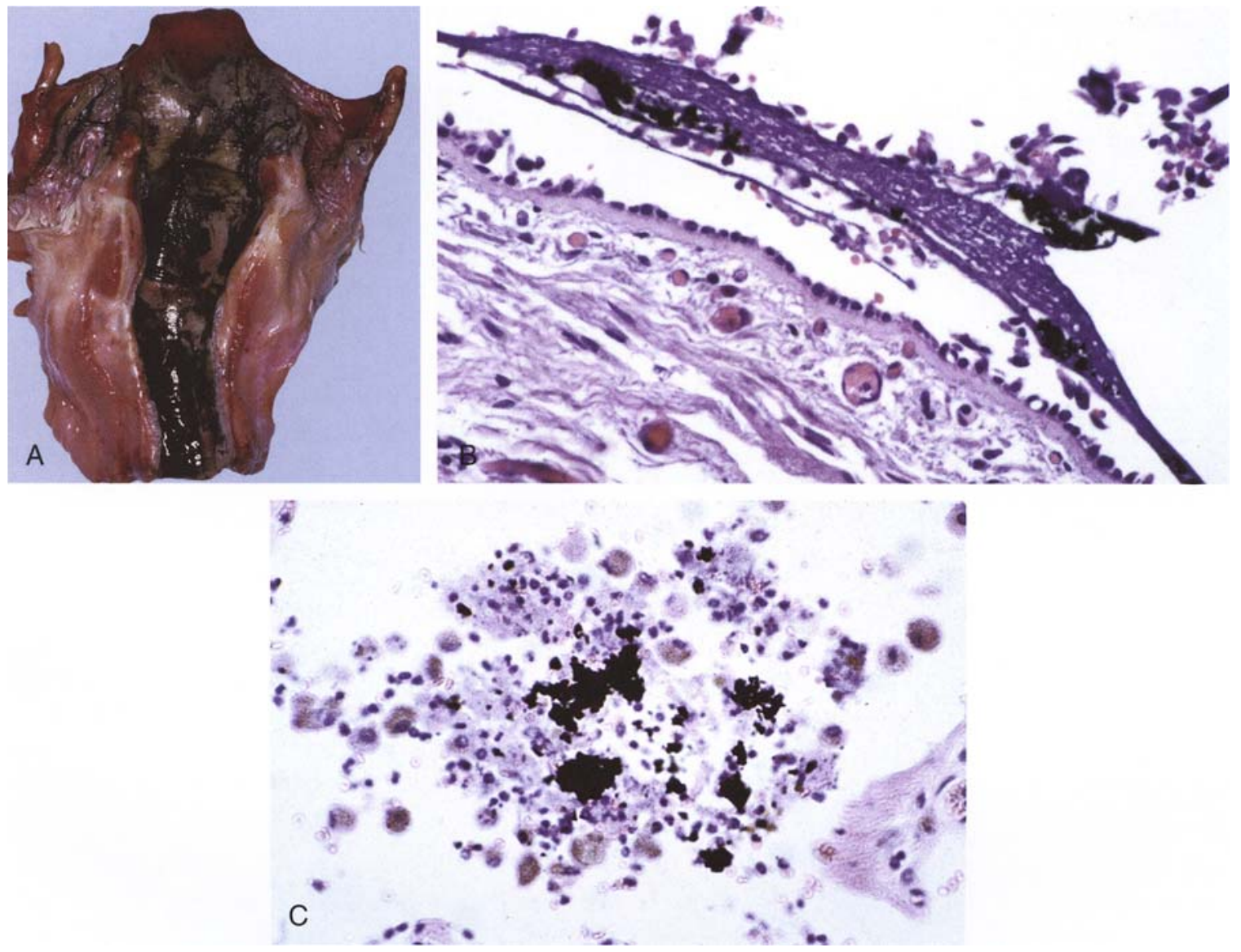

Figure 31.46. Smoke inhalation. A. Soot in airway. B. Soot particles mixed with mucus overlying partially denuded airway mucosa. C. Soot particles in alveolar space with macrophages and neutrophils.

\section{Terrorism}

Whereas in the past, the evaluation of persons affected by biologic or chemical warfare agents was essentially restricted to military personnel, it is now apparent that civilian pathologists, both hospital and forensic, will be faced with evaluating persons who have become ill or have died as the result of what may be suspected to have been exposure to biologic or chemical agents released during terrorist activity. Although there are almost countless chemicals, organisms, and toxins that may harm humans, those that are most likely to be adaptable to nefarious widespread dissemination are limited. The use of some of these agents is intended to kill, while others are intended to incapacitate. Portals of entry are typically respiratory, cutaneous/mucousmembrane,orgastrointestinal.Although exposure may be inhalational in many instances, the clinical and pathologic manifestations may primarily involve other systems, with respiratory lesions being minor. These agents and agents causing death through central nervous system (CNS) or neuromuscular respiratory depression or paralysis (e.g.,nerve agents) will not be discussed. However, several chemical and biologic agents result in lesions that prominently involve the chest.

Some agents typically cause the rapid development of respiratory casualties (Table 31.5), while casualties caused by other agents are usually delayed (Table 31.6). Overall, the rapid onset of respiratory casualties is seen following exposure to nerve agents, vesicants, respiratory agents, and staphylococcal enterotoxin B (SEB) inhalation. Delayed-onset respiratory casualties are caused by inhalational anthrax, pneumonic plague, pneumonic tularemia, Q fever, brucellosis, melioidosis, SEB inhalation, ricin inhalation, vesicants, and phosgene. ${ }^{379}$

TABLE 31.5. Rapid onset respiratory casualties-chemical and biologic agents

Nerve agents

Vesicants

Respiratory agents

Staphylococcal enterotoxin inhalation

Source: Data from U.S. Army Medical Research Institute of Infectious Diseases. $^{379}$ 
TABLE 31.6. Delayed onset respiratory casualties-chemical and biologic agents

Inhalational anthrax

Pneumonic plague

Pneumonic tularemia

Q fever

Brucellosis

Melioidosis

Staphylococcal enterotoxin inhalation

Ricin inhalation

Vesicants

Phosgene

Source: Data from U.S. Army Medical Research Institute of Infectious Diseases. ${ }^{379}$

Detailed discussion of each of the chemical and biologic agents is beyond the scope of this chapter. For detailed description and discussion of particular agents, refer to the appropriate chapters in this book or other publications. However, brief mention of some of these agents is warranted.

\section{Chemical Agents}

Among the chemical agents that damage the respiratory tract (Table 31.7), perhaps we have the most historical experience with exposure to vesicant agents, notably sulfur and nitrogen mustard. Other vesicant agents include Lewisite and phosgene oxime. ${ }^{380}$ These alkylating agents are slowly soluble in water but then rapidly form cyclic ethylene sulfonium ions that are highly reactive with cell proteins, cell membranes, and DNA in rapidly dividing cells. ${ }^{380}$ The result is direct exposure dose-dependent epithelial necrosis. For this reason, airway damage is typically most severe in the proximal airway. Examination of the airway demonstrates necrosis of the epithelium. Inflammatory pseudomembrane formation is prominent. ${ }^{380}$ The lung parenchyma is usually initially normal unless exposure was heavy. ${ }^{381}$ The death rate is $3 \%$. Severe exposures may result in deaths in the first 24 hours caused by airway obstruction due to the pseudomembranes and laryngospasm. Most deaths occur after the fourth day and are caused by bacterial superinfection with pneumonia, or, 1 to 3 weeks after exposure, by sepsis related to agent-induced bone marrow suppression.

A host of respiratory sequelae $e^{382-386}$ to mustard inhalation have been reported including tracheobronchial stenosis $^{386}$ and long-term sensitivity to respiratory irritants. ${ }^{387,388}$ Inhalational and systemic exposure to phosgene oxime have each been associated with the development of pulmonary edema. This agent has been reported to cause pulmonary venule thrombosis and necrotizing bronchiolitis. ${ }^{380,389}$

In contrast to the highly reactive vesicants, other agents are relatively insoluble and nonreactive with moist epi- thelial surfaces. These agents relatively spare the proximal airway and exert their effects in the lung parenchyma. Phosgene is the prototypical respiratory agent. Other agents that may be encountered primarily on the battlefield include perfluoroisobutylene (PFIB) and nitrogen oxides $\left(\mathrm{NO}_{\mathrm{x}}\right)$. Phosgene is inhaled and carried to the alveolar-capillary membrane where acylation reactions cause damage that leads to the development of pulmonary edema. The proximal airway is usually spared unless there has been heavy exposure. Proximal airway epithelial damage during heavy exposure is related to the release of $\mathrm{HCl}$ by the phosgene.

\section{Biologic Agents}

Biologic agents potentially useful for terrorism or warfare include a variety of bacteria, viruses, rickettsia, and toxins (bacterial and fungal). Most of these agents and the diseases they cause are discussed in the chapters on infectious diseases and are not discussed here except for a brief discussion of two toxins. There are a variety of publications offering comprehensive discussions of the biologic warfare and terrorism aspects of these agents. ${ }^{390,391}$

\section{Staphylococcal Enterotoxin B}

Staphylococcal enterotoxin B (SEB) is one of seven enterotoxins produced by Staphylococcus aureus. Although commonly a causative agent in food poisoning, nonenteric exposure to staphylococcal enterotoxins can result in serious illness. ${ }^{380}$ The SEB disorders are mediated by massive release of cytokines including interferon$\gamma$, IL-6, and TNF- $\alpha$. The effects of inhaling SEB are more likely to be incapacitating than lethal. There are limited data describing the effects of SEB inhalation in humans. A laboratory SEB inhalation accident resulted in exposure of at least nine persons. These individuals became ill 5 to 16 hours following exposure. Signs and symptoms persisted for 50 to 130 hours and included cough, shortness of breath, rales, substernal pleuritic chest pain, fever, and headache. Some chest radiographs demonstrated pulmonary edema. Severity of illness was rated as mild

TABLE 31.7. Chemical agents having pulmonary effects

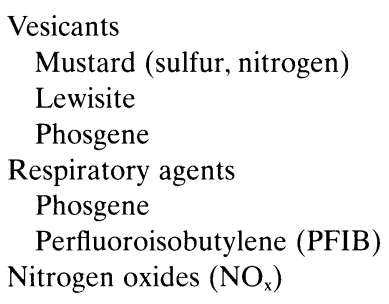

Source: Data from Sidell et al. ${ }^{380}$ 
(four cases), moderate (two cases), or severe (three cases). All of the individuals recovered with sequelae. ${ }^{380}$

Pulmonary findings were evaluated in a series of monkeys with inhalational exposure to SEB. The animals developed gastrointestinal dysfunction within 24 hours of exposure followed by the sudden onset of lethargy, dyspnea, and pallor at 48 hours. The animals died or were euthanized within the subsequent 4 hours. Respiratory tract necropsy findings included edema, acute alveolitis, diffuse alveolar damage, and inconsistent loss of airway epithelium. Respiratory lymphoid tissue showed B-cell depletion and T-cell hyperplasia. ${ }^{380}$

\section{Ricin}

Ricin is a lectin found in the castor plant bean (Ricinis communis) ${ }^{380}$ Descriptions of the effects of acute inhalation by humans are lacking. ${ }^{380}$ Inhalation studies in monkeys feature the development of extensive fibrinopurulent pneumonia, pulmonary edema, and variable airway necrosis and inflammation. Mediastinal purulent lymphadenitis was severe. ${ }^{392}$ Some workers in castor plant commercial processing facilities who are chronically exposed to inhaled ricin have developed an allergic syndrome. ${ }^{393}$

\section{Substance Abuse}

The use of illicit psychoactive substances may result in a variety of primary and secondary lesions involving all components of the respiratory tract. ${ }^{394}$ The extensive highly absorptive surface area of the lung makes it an ideal entry portal for aerosolized, volatilized, and pyrolized substances. As the first capillary bed encountered following systemic venous injection, the lung serves as the major entrapment point of insoluble particulates.

Some effects of abused substances on the respiratory structures are dictated by the pharmacologic properties of the chemical and are influenced by the route, rate, and chronicity of administration. Additional effects may reflect the presence and characteristics of filler/ carrier material, adulterants, or contaminants (chemical or biological); methods of preparation; and route of administration.

Recognition of changes indicative of a particular route of administration, even though they do not establish the cause of death, may be important in explaining the significance of other findings or to corroborate statements by witnesses. The presence of certain findings can be useful in establishing a particular mode of administration, but the lack of these findings is not particularly helpful in excluding potential routes of administration. It must also be recognized that a particular person may employ multiple routes of administration.

As with any administered substance, the presence of the substance or its metabolites only establishes exposure and does not necessarily indicate the presence of drugrelated disease or that the drug caused or contributed to death. The significance of the drug or drug-related disease in a particular person can be established only by interpreting the findings in the context of the circumstances of death.

Infectious complications commonly arise as a consequence of substance abuse, especially when the route of administration is parenteral. Infectious complications are not discussed in this chapter, and the reader is referred to the appropriate chapters on lung infections for discussions of these particular diseases (e.g., HIV-related conditions, pneumonia).

Currently, the most commonly abused substances having pulmonary effects are the stimulants (cocaine, amphetamines), opiates/narcotics (primarily heroin, oxycodone, fentanyl), and cannabinoids (marijuana). The remainder of this section discusses features of each of these groups of substances, followed by a discussion of the lesions attributed to them.

\section{Cocaine}

Cocaine, derived from the coca plant, is the most common cause of illicit substance abuse deaths in the United States and, except for marijuana, is arguably the most popular of the illicit substances. ${ }^{395}$ In 1999 there were an estimated 1.5 million cocaine users in the United States, of which 413,000 used the "crack" form of the substance. ${ }^{395}$ The rate of use was highest among the 18- to 25-year age group.

Cocaine is obtainable in a number of forms depending on processing and packaging techniques. Among these cocaine compounds are coca leaf, coca paste, cocaine hydrochloride, and the cocaine alkaloid (freebase or crack). While cocaine hydrochloride has been the most common form of the drug available in the United States in the past, crack cocaine is currently the most widely found form in many parts of the country.

In past years, cocaine hydrochloride bought on the street was diluted (cut) with a variety of compounds (generally water soluble), resulting in relatively low concentrations of cocaine in the retail product. The product currently sold at street level generally contains $50 \%$ to $80 \%$ cocaine. ${ }^{395}$ The crack form of cocaine available on the street is essentially pure cocaine base.

Processing of cocaine involves taking the cocaine leaf and submitting it to a number of steps involving the use of water, gasoline, and acid to form a paste that is then further processed to cocaine hydrochloride. The cocaine hydrochloride may be further processed to yield the alkaloid (base) form.

There are two general methods for transforming the cocaine hydrochloride into its alkaloid (base) form: freebase or crack. Although these forms are chemically 
identical, these terms traditionally have been applied to the end result of different processing techniques. Freebase cocaine results from extraction of the alkaloid by using ammonia and ether. The crack cocaine technique involves the use of water, baking soda, and heat. ${ }^{395}$

Cocaine can be administered in a variety of ways. Coca leaf is usually chewed and coca paste can be smoked. These forms of cocaine are unusual in the United States. Typically, in the United States, cocaine is inhaled (generally by smoking), insufflated, or injected (usually intravenously). The preferred route of administration is generally inhalation or insufflation. In addition to personal preference, the form of cocaine being used influences the route of administration. Cocaine hydrochloride is water soluble and undergoes thermal decomposition, thus making smoking ineffective. In contrast, the water-insoluble alkaloid forms of cocaine vaporize when heated and are preferentially administered by smoking. The effects of smoking the alkaloid forms of cocaine are akin to those seen following intravenous injection of the drug. ${ }^{1}$

Respiratory signs and symptoms have been reported in $20 \%$ to $60 \%$ of alkaloid cocaine smokers. The respiratory effects of cocaine include local airway irritation and vasoconstriction, barotrauma, parenchymal lesions, and vascular adaptations. ${ }^{395}$

\section{Other Stimulants}

Methamphetamine is a synthetic stimulant responsible for a small, but growing percentage of drug-related emergencies and deaths. Much of the drug is synthesized in small clandestine "meth labs." More recently, a significant amount of the drug originates from large "super labs" or from overseas production facilities. ${ }^{395}$

In the United States, most methamphetamine is synthesized from either ephedrine or pseudoephedrine by reductive halogenation using red phosphorus as a catalyst. ${ }^{395}$ Until recently, ephedrine was easily obtained from a variety of vitamin, dietary supplement, or organic food vendors. Currently, the demand for ephedrine to be used in the production of methamphetamine has resulted in the government restricting sales and access to ephedrine. Similarly, strict controls have been placed on the sales of a number of the other chemicals needed in the synthesis process. Lack of availability of ephedrine has led the "meth cooks" to substitute a variety of cold remedies for the ephedrine-mixtures of diphenhydramine, chlorpheniramine, dextromethorphan, doxylamine, and guaifenesin. Methamphetamine production generates many products, some of which are strong stimulants and others having various toxic effects. ${ }^{395}$

Methamphetamine may be successfully administered by swallowing, injection, smoking, or snorting. Most users either inject intravenously or swallow methamphetamine ${ }^{396}$ However, crystalline methamphetamine ("ice") formed by slow crystallization of methamphetamine oil by refrigeration may also be smoked (often by inhaling vapors emanating from the screen of a "speed pipe"). ${ }^{395}$

\section{Heroin/Narcotics}

Heroin toxicity accounts for about $40 \%$ of reported drug deaths. ${ }^{395}$ Recently, usage of and deaths due to previously uncommon narcotics have been increasing-notably hydrocodone, oxycodone, methadone, and fentanyl. While common in the past, deaths due to the injection of pentazocine have all but disappeared due, in part, to pharmacologic engineering that incorporated a narcotic antagonist that is rapidly hydrolyzed by gastric acid into the oral formulation of the drug. Appropriate administration of the drug (oral) resulted in absorption of the drug and hydrolysis of the antagonist in the stomach, allowing the drug to be systemically effective. Inappropriate administration (injection) negated the effect of the drug due to the concomitant presence of the functional antagonist.

Heroin (diacetyl morphine) can be insufflated (snorted), inhaled (either smoked or via inhaling the vapors, the latter known as "chasing the dragon"), or injected (subcutaneous or intravenous) (Fig. 31.47). Heroin is ineffective when ingested due to rapid hydrolysis in the stomach. Other narcotics such as morphine and methadone are effective when ingested.

Preparations designed for transdermal use (e.g., fentanyl transdermal patches) may be abused in that fashion but can also be ingested (chewed) or smoked. Extracts prepared from transdermal systems are often injected. ${ }^{397}$

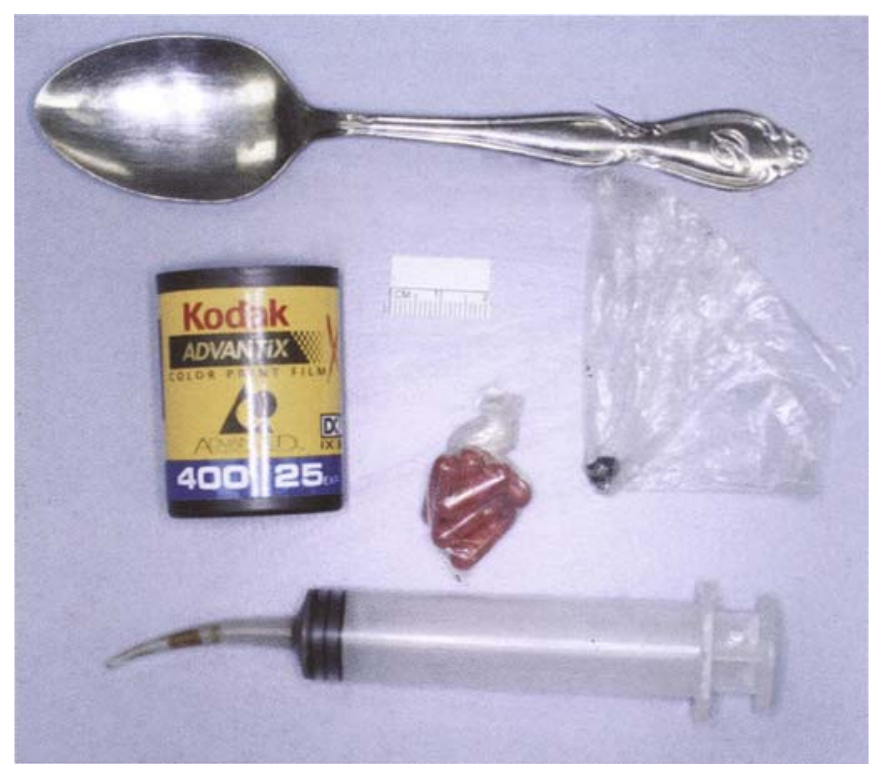

FIGURE 31.47. Kit to prepare heroin for injection. 
Oral preparations of various types can be ingested or injected.

\section{Cannabinoids}

The most widely used illicit substances in the world are cannabinoids. These are most commonly obtained in preparations of marijuana and, to a lesser extent, hashish. The primarily psychoactive agent, $\delta$-9-tetrahydrocannabinol $(\delta-9-\mathrm{THC})$ is found in the flower or fruiting tops, leaves, and resin of the Cannabis sativa plant. The plant components are dried and then usually consumed by smoking. Occasionally, the plant components may be made into foodstuffs and ingested (e.g., "hash brownies").

As noted above, there are several respiratory tract lesions associated with substance abuse. None of these are specific for any particular drug, although some substances or routes of administration are more commonly associated with particular lesions.

\section{Respiratory Manifestations}

\section{Airways}

Upper airway lesions are primarily associated with insufflation of vasoactive substances, notably cocaine, and smoking. Perforation of the nasal septum is a wellrecognized complication of chronic insufflation of cocaine presumably mediated through vasoconstriction-induced ischemia. Submucosal arteriolar intimal hyperplasia and fibrosis and perivascular fibrosis have been observed in the nasal septa of cocaine abusers. ${ }^{395}$ Upper respiratory epithelial necrosis, also presumably related to ischemia, has also been associated with the inhalation of cocaine. ${ }^{395}$

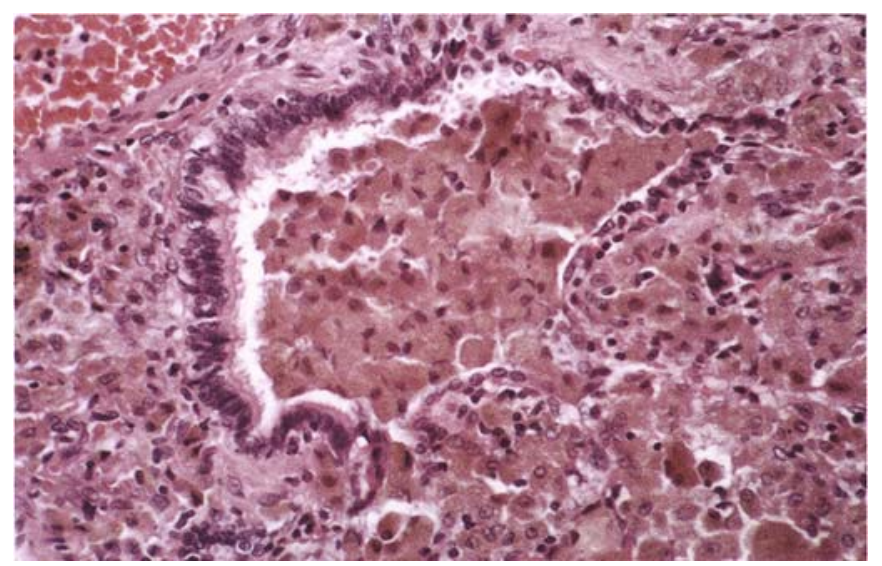

FiguRE 31.48. Pigmented macrophages in and around respiratory bronchiole in a marijuana smoker. (From Tomashefski and Felo, ${ }^{394}$ with permission from Elsevier. Copyright $\odot$ 2004.)

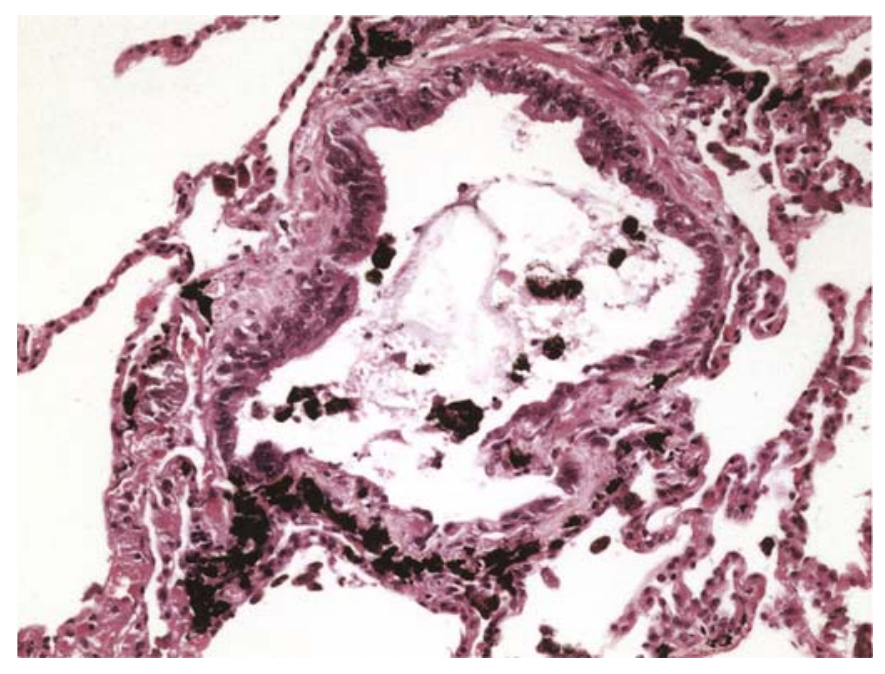

Figure 31.49. Black particulate material in membranous bronchiole of a cocaine smoker.

Thermal burns have infrequently complicated crack smoking. Extensive burns to the face and upper aerodigestive tract have occurred with freebase smoking. These are likely caused by the ignition of residual ether remaining from processing. Thermal burns have also been attributed to hot particulates escaping from inadequately filtered pipes and from aspiration of dislodged hot wire mesh or steel wool used for filtering in crack pipes. ${ }^{398-402}$

Combustion products of Cannabis sativa, and to a lesser extent cocaine, are respiratory irritants. Many of the changes attributed to marijuana smoking are similar to those associated with tobacco smoke and are difficult to assess in individual cases due to the common habit of marijuana smokers of also abusing tobacco. However, it appears that marijuana smoking alone causes significant pulmonary changes faster than tobacco smoking alone. Marijuana smoke surpasses tobacco smoke in carboxyhemoglobin production and in both the inhalation and retention of tar. ${ }^{403-407}$ The proximal airways of marijuana and cocaine smokers have shown basal and goblet cell hyperplasia, squamous metaplasia, epithelial atypia, and basement membrane thickening. Marijuana smokers also developed lower airway changes similar to those of tobacco smokers-increased pigmented alveolar macrophages (Fig. 31.48) ${ }^{408}$ respiratory bronchiolitis, and goblet cell hyperplasia. Chronic marijuana smoking has been associated with chronic bronchitis and declines in pulmonary function.

The sputum and lungs of crack smokers often contain coarse, irregular carbonaceous particulates that are extracellular and within alveolar macrophages (Fig. 31.49). These may impart a black color to the sputum, a phenomenon reported in nearly $44 \%$ of crack smokers. Much of 
this material may reflect the habit of some crack smokers to remove and smoke the black residual material that accumulates in the crack pipe. ${ }^{409}$ Some crack smokers are under the mistaken notion that this residual material contains concentrated cocaine.

\section{Asthma}

A relationship between cocaine, especially when inhaled, and asthma has been described. ${ }^{410-412}$ Crack smoking is occasionally accompanied by wheezing. ${ }^{413,414}$ It appears that the irritant effect of cocaine smoke can exacerbate preexisting asthma. ${ }^{42,415}$ Cocaine does not appear to cause nonspecific airway hyperresponsiveness ${ }^{416}$ or chronic asthma.

There is no specific feature that distinguishes a cocaine-related asthma death from a death unrelated to cocaine. Because it is the irritant effect of cocaine smoke that appears to be responsible for initiating asthma attacks, the attack should begin within a relatively short time following the inhalation of the cocaine. Information from the history or toxicologic information may establish the temporal relationship between the cocaine use and the asthma attack. ${ }^{1}$ Heroin has occasionally also been associated with exacerbation of asthma. ${ }^{417}$ This may be due to release of histamine or central respiratory depression.

\section{Allergic Bronchopulmonary Fungal Disease}

Smoking mold-contaminated marijuana may lead to allergic bronchopulmonary fungal disease. Aspergillus, mucor, penicillium, and thermophilic actinomycetes have been recovered from marijuana samples. ${ }^{418,419}$ Kagen et al. ${ }^{418}$ reported finding precipitating antibodies to aspergillus in $57 \%$ of marijuana smokers and blastogenesis in $13 \%$. Fungal elements have been observed in marijuana smokers who have developed allergic bronchopulmonary aspergillosis and necrotizing granulomas. ${ }^{419-421}$

\section{Bronchiectasis}

Diffuse or localized cylindrical and varicose bronchiectasis has been reported as a late sequela of heroin use. ${ }^{422}$ Although the bronchiectasis is related to aspiration and bronchiolitis in some cases ${ }^{423}$ in other cases its development has been reported to be unrelated to aspiration. ${ }^{422}$

\section{Neoplasia}

Predisposition to respiratory neoplasia may be related to marijuana and, to a lesser extent, cocaine smoking. ${ }^{424,425}$ The type and amount of carcinogens in marijuana smoke are equivalent to those present in tobacco smoke. ${ }^{424,425}$ Marijuana and cocaine smokers demonstrate significant molecular alterations that are linked to an increased cancer risk in the bronchial mucosa. ${ }^{425}$

\section{Pulmonary Edema}

Pulmonary edema is a common nonspecific finding at autopsy, especially if cardiopulmonary resuscitation has been attempted. However, the development of pulmonary edema is a well-recognized consequence of narcotic intoxication and has been associated less frequently with the use of stimulants.

Marked pulmonary congestion, often, but not invariably, associated with exuberant pulmonary edema, is a hallmark of fatal narcotic intoxication and is primarily caused by profound respiratory depression with associated hypoxia. ${ }^{395}$ Other proposed but not generally accepted mechanisms include direct toxicity and hypersensitivity. ${ }^{426}$ The pulmonary edema may be exacerbated by gastric aspiration that frequently complicates narcoticinduced unconsciousness. ${ }^{426}$ Pulmonary edema fluid resulting from opiate intoxication characteristically is protein-rich ${ }^{394}$ and is often whipped into a prominent froth during agonal breathing.

The lungs of persons dying of opiate intoxication are typically heavy (in excess of $1400 \mathrm{~g}$ in toto), voluminous, congested, and edematous. ${ }^{427}$ The edema is usually, but not always, prominent. Foamy edema fluid often fills the airways and may exude from the nose and mouth (Fig. 31.50A). It may be blood-tinged (Fig. 31.50B). Histologically, there is interlobular septal and perivascular expansion with edema fluid. Intravascular leukostasis and acute alveolitis have been described in some cases. ${ }^{427,428}$

Pulmonary edema associated with stimulant use is not clearly defined. In some cases, if not most, it is related to left ventricular dysfunction or increased pulmonary vascular pressure. ${ }^{409,429}$ Other proposed mechanisms include increased permeability and increased central nervous system activation (neurogenic pulmonary edema). Few cases in which noncardiogenic pulmonary edema has been suggested have supporting hemodynamic data.

\section{Pulmonary Hemorrhage}

Pulmonary hemorrhage is a well-recognized complication of both alkaloid cocaine use $e^{409,430}$ and opiate intoxication. Acute hemorrhage and hemosiderin-laden macrophages (Fig. 31.51) have been observed in 58\% and $40 \%$, respectively, of individuals in an autopsy study including persons testing positive for cocaine. ${ }^{431}$ Crack users occasionally develop hemoptysis. Hemosiderinladen macrophages have been observed in BAL fluid and open lung biopsy in a crack smoker. ${ }^{431,432}$ Recurrent hemoptysis temporally related to multiple episodes of freebase smoking has been described in an individual. ${ }^{433}$

Potential mechanisms for cocaine-induced alveolar hemorrhage include reperfusion hemorrhage following vasoconstriction-induced ischemic damage, direct toxicity, and hypersensitivity reaction. ${ }^{430,431}$ Activation of neutrophils and enhanced production of IL-8 following 

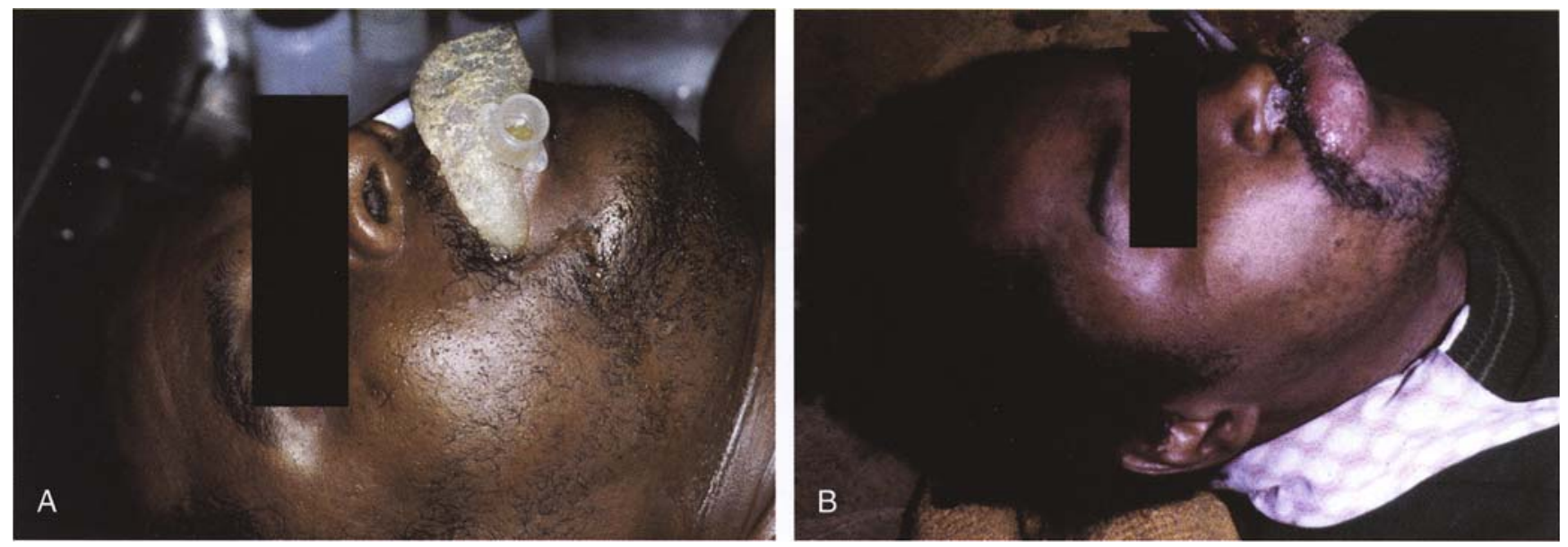

Figure 31.50. Heroin-associated pulmonary edema. A. Foam cone. B. Blood-tinged foam cone.

short-term cocaine use suggests a role for acute inflammation in the development of lung injury. ${ }^{434}$ However, capillaritis is not a prominent histologic feature. ${ }^{409}$ Cardiac compromise may also play a role in cocaine-induced pulmonary hemorrhage. ${ }^{431}$

Although gross hemoptysis is not frequent in opiate intoxication, some pulmonary hemorrhage and hemosiderin- laden macrophages can be seen in deaths due to opiate intoxication. This likely reflects extravasation associated with episodes of pulmonary congestion.

\section{Interstitial Lung Disease}

Occasional instances of interstitial lung disease unrelated to embolic particulates are associated with substance abuse, primarily alkaloid cocaine smoking. ${ }^{410,424,435,436}$

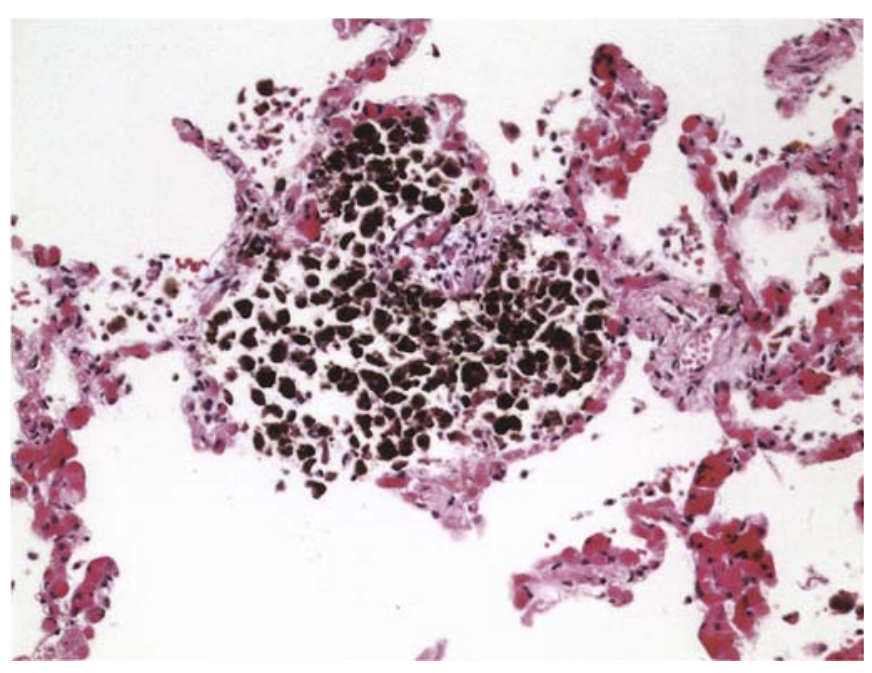

FIGURE 31.51. Cocaine-associated lung hemorrhage. Numerous alveolar hemosiderophages.
Inflammatory infiltrates associated with fever, hypoxia, hemoptysis, and, occasionally, respiratory failure following alkaloid cocaine inhalation have been referred to as "crack lung." ${ }^{436}$ Most of these are self-limited, of short duration, and resolve without serious sequelae. The cases originally described feature mild interstitial pneumonitis, rare eosinophils, and type II pneumocyte hyperplasia. ${ }^{436}$ Subsequently, a broader spectrum of changes has been described: nonspecific interstitial pneumonia and fibrosis, diffuse alveolar damage, alveolar hemorrhage, bronchiolitis obliterans-organizing pneumonia (BOOP), and a pattern resembling acute eosinophilic pneumonia. ${ }^{430,431,437}$

\section{Aspiration}

The effects of gastric aspiration are commonly seen in cases of opiate use in which the user becomes unconscious. Occasionally, milk aspiration may be seen in heroin users to whom the milk has been administered due to the mistaken notion that it is an effective oral antidote for opiate intoxication. ${ }^{394}$ Purulent bronchitis, bronchopneumonia (suppurative or granulomatous), bronchiolitis obliterans, and bronchiectasis may be seen in individuals who survive the initial aspiration episode. ${ }^{423,427}$

\section{Vascular}

Vascular lesions associated with substance abuse are most commonly due to the introduction of insoluble particulates during intravenous injection. The direct effects of some drugs, notably sympathomimetic agents, may also affect the vasculature.

\section{Embolic Lesions}

The appearance of particulate material and other pulmonary findings associated with substance abuse may be 

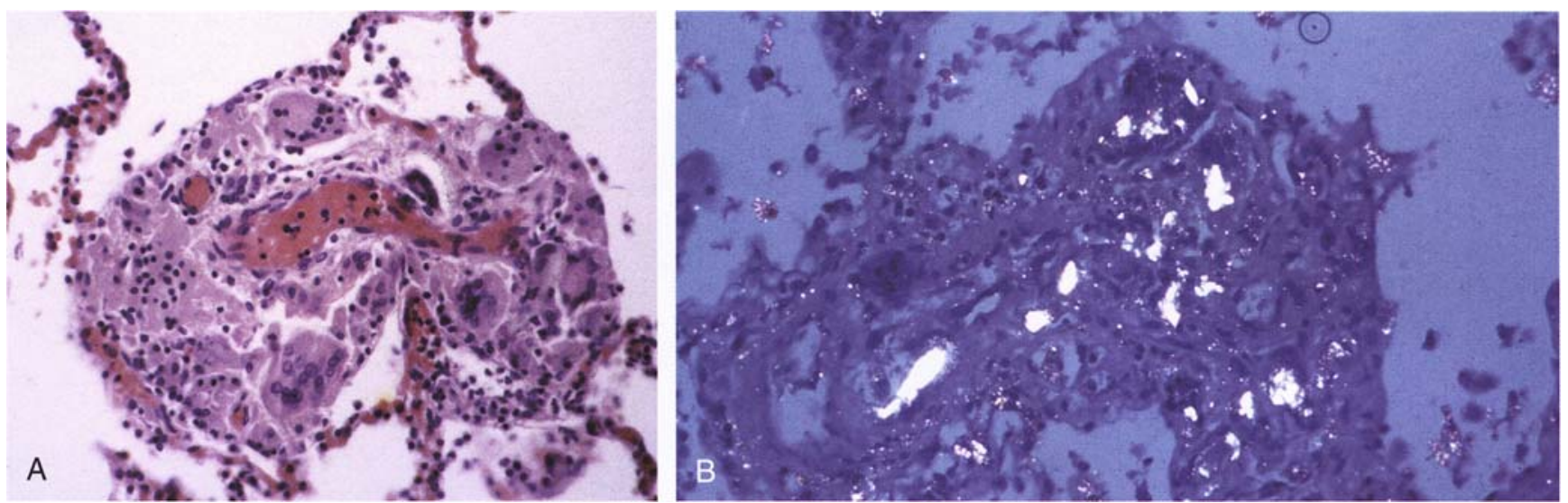

Figure 31.52. Chronic intravenous narcotism. A. Vascular effects of foreign material injection. Foreign-body giant cells surround and infiltrate the wall of pulmonary arteriole. B. Vas-

cular and perivascular deposition of birefringent oral pharmaceutical filler material (polarized light). route of administration is known either by information from the history or through other findings (e.g., injection sites). Occasionally, the presence of substance abuse or the route of exposure is unsuspected, concealed, or in dispute. Once the presence of material that may be medication filler is established, a major issue that needs resolution is whether the material was inhaled or injected (usually intravenously). A number of factors generally allow this distinction to me made. These factors include the location of the material and any reaction to it, the state of ferrugination among certain types of particles, and the size distribution of the particles. The presence of a foreign-body reaction within the blood vessel wall or

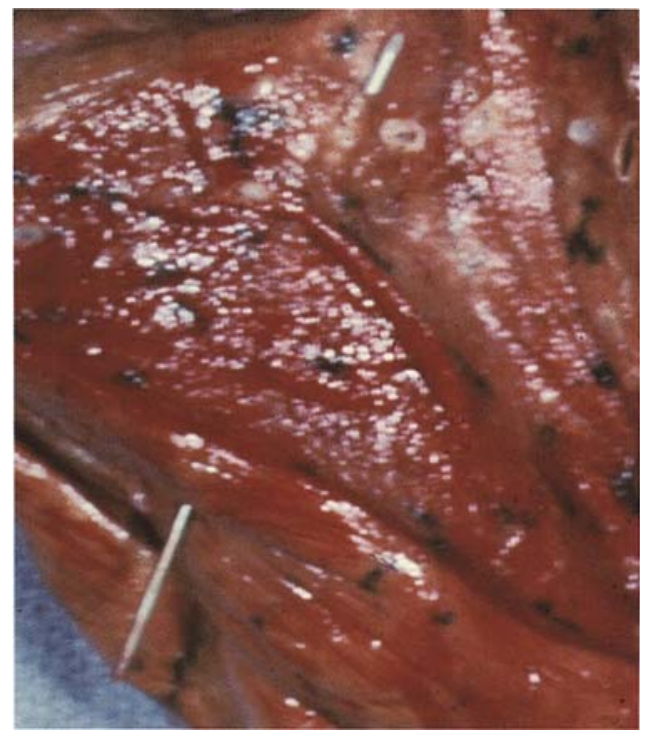

FIGURE 31.53. Lung with migratory needle that broke off during central venous injection. may be a critical issue in a particular case. Usually, the 
lumen is indicative of injection. In the case of talc, inhaled particles often show some evidence of ferrugination, whereas injected particles do not tend to ferruginate. Uncoated talc particles are generally absent from air spaces in cases of intravenous injection. ${ }^{1}$ Inhaled particles are generally no more than $5 \mu \mathrm{m}$ in diameter, whereas injected substances have a substantial population of larger particles. ${ }^{442}$ Further discussion of the effects of insoluble particulate emboli is presented in Chapter 26.

\section{Nonembolic Lesions}

Nonembolic vascular changes may also occur. Murray et al. ${ }^{443}$ identified muscular pulmonary artery medial hypertrophy in $20 \%$ of individuals whose deaths were associated with alkaloid cocaine. It has been suggested that crack smokers may develop pulmonary vasospasm. Pulmonary artery smooth muscle hypertrophy and elastic fiber proliferation have been observed and attributed to excessive stimulation of vascular $\alpha_{1}$-adrenergic receptors. Clarification of this issue awaits further data.

It is unclear if substance abuse is associated with the development of plexiform pulmonary hypertension. ${ }^{444}$ The anorexogenic agent aminorex fumarate has been associated with an epidemic of plexiform pulmonary hypertension. This agent has structural similarities to cocaine and amphetamines. Schaiberger et al. ${ }^{445}$ reported a case of pulmonary hypertension developing in a long-term methamphetamine inhaler; however, this case lacks histologic confirmation. If stimulant abuse is associated with the development of plexiform pulmonary arteriopathy, the incidence must be quite low and other causes should be scrupulously excluded prior to attributing the disease to the pharmacologic effects of stimulant abuse. ${ }^{395}$

\section{Barotrauma}

Pulmonary barotrauma can occur as a consequence of substance abuse. It is most commonly associated with cocaine and marijuana smoking. ${ }^{446-448}$ Increases in alveolar pressure or decreases in interstitial pressure lead to alveolar rupture and dissection of air into the mediastinum or pleura.

Increased alveolar pressure is usually due to coughing or performing the Valsalva maneuver. ${ }^{446}$ Occasionally, positive pressure ventilation is provided by a companion forcibly breathing more smoke into the recipient's lungs. ${ }^{447}$ Other proposed mechanisms include inflammation leading to weakened alveolar walls and decreased interstitial pressure related to vasoconstriction.

Drug-related barotrauma is consistently associated with pleuritic chest pain and dyspnea. Barotrauma usually spontaneously resolves and treatment is symptomatic. The author is not aware of any deaths that have been attributed to marijuana or cocaine-induced barotrauma.

\section{Bullous Lesions}

Giant bullae have been observed in marijuana and cocaine smokers as well as in intravenous substance abusers. ${ }^{44-451}$ The bullae are usually apical and have the same appearance as those associated with idiopathic spontaneous pneumothorax. ${ }^{395}$ Mechanisms proposed to explain the formation of these bullae include tobacco and possibly non-tobacco-related emphysema, talc-induced emphysema, and recurrent barotrauma. ${ }^{449-451}$

\section{Other Issues}

A variety of medicolegal issues may arise regarding exposures to potentially injurious agents encountered in the workplace or during medical care (e.g., drug toxicity, radiation exposure). Issues in these cases typically center around worker/patient safety, personal injury liability, and wrongful death.

A discussion of these entities is beyond the scope of this chapter. The reader is referred to the appropriate chapters in this text for more detailed discussion.

\section{Testifying as a Witness}

The pathologist may be called to testify in a number of capacities in legal proceedings, most often as an expert witness. As an expert due to his or her special knowledge, experience, training, or education, the pathologist's opinions are sought to help the trier of fact (jury or judge) understand the pathologic evidence or issues pertinent to the case.

A discussion of the pathologist's role and duties in the legal system is beyond the scope of this chapter. There are a number of publications devoted to this topic. Some of these publications are written by and for pathologists. ${ }^{452,453}$

\section{Conclusion}

The pathology of the respiratory tract plays an important role in many forensic issues greatly affecting decedents' families and estates, public health, civil litigation, and the criminal justice system.

\section{References}

1. Graham MA, Hutchins G. Pulmonary Disease. Forensic pathology. Clin Lab Med [Part II] 1998;18(2):241-262.

2. Hanzlick R, Hunsaker JC, Davis GJ. A guide for manner of death classification. Atlanta: National Association of Medical Examiners, 2002.

3. Shah RK, Roberson DW, Jones DT. Epiglottitis in the Haemophilus influenzae type $\mathrm{B}$ vaccine era: changing trends. Laryngoscope 2004 114(3):557-560. 
4. Wenger JD. Epidemiology of Haemophilus influenzae type $B$ disease and impact of Haemophilus influenzae type B conjugate vaccines in the United States and Canada. Pediatr Infect Dis J 1998;17:S132-136.

5. Howell NR. Sudden death from laryngeal papillomatosis. Am Soc Clin Pathol Check Sample 2005;47(4):41-51.

6. Fyfe B, Mittleman R. Hypopharyngeal lipoma as a cause of sudden asphyxial death. Am J Forensic Med Pathol 1991;12:82-84.

7. Dada MA. Laryngeal cyst and sudden death. Med Sci Law 1995;35:72-74.

8. Gardner P, Jentzen J, Komorowski R et al. Asphyxial death caused by a laryngeal schwannoma: a case report. J Laryngol Otol 1997;111:1171-1173.

9. Taff M, Schwartz I, Boglioli L. Sudden asphyxial death due to a prolapsed esophageal fibrolipoma. Am J Forensic Med Pathol 1991;12:85-88.

10. DiMaio VJ, DiMaio D. Forensic pathology, 2nd ed. Boca Raton, FL: CRC Press, 2001.

11. Berry CL. Causes of sudden natural death in infancy and childhood. In: Mason JK, ed. Paediatric forensic medicine and pathology. London: Chapman and Hall Medical, 1989.

12. O'Shea PA. Congenital and acquired systemic infectious diseases. In: Stocker JT, Dehner LP, eds. Pediatric Pathology. Philadelphia: JP Lippincott, 1992.

13. Valdes-Dapena M, McFeeley PA, Hoffman HJ, et al. Histopathology atlas for the sudden infant death syndrome. Washington, DC: Armed Forces Institute of Pathology, 1993.

14. Handy T, Buchino J. Sudden natural death in infants and young children. In: Froede RC, ed. Clin Lab Med 1998; 18(2):323-338.

15. Church NR, Anas NG, Hall CB, Brooks JG. Respiratory syncytial virus-related apnea in infants. Am $\mathrm{J}$ Dis Child 1984;138:247-250.

16. Anas N, Boettrich C, Hall CB, Brooks JG. The association of apnea and respiratory syncytial virus infection in infants. J. Pediatr 1982;101:65-68.

17. Bruhn FW, Mokrohisky ST, McIntosh K. Apnea associated with respiratory syncytial virus infection in young infants. J Pediatr 1977;90:382-386.

18. Adams JM. Primary virus infection with cytoplasmic inclusion bodies. Study of an epidemic involving thirty-two infants, with nine deaths. JAMA 1947;116:925-933.

19. Adams JM, Imagawa DT, Zike K. Epidemic bronchiolitis and pneumonitis related to respiratory syncytial virus. JAMA 1967;176:1037-1039.

20. Neilson KA, Yunis EJ. Demonstration of respiratory syncytial virus in an autopsy series. Pediatr Pathol 1990; 10:491-502.

21. Sidebotham HJ, Roche WR. Asthma deaths: persistent and preventable mortality. Histopathology 2003;43:105117.

22. Weitzman JB, Kanarek NF, Smialek JE. Medical examiner asthma death autopsies; a distinct subgroup of asthma deaths with implications for public health preventive strategies. Arch Pathol Lab Med 1998;122:691-699.

23. Turner-Warwick M. Epidemiology of nocturnal asthma. Am J Med 1988:85(suppl 1B):6-8.
24. Greenberger PA, Miller TP, Lifschultz B. Circumstances surrounding deaths from asthma in Cook County (Chicago) Illinois. Allergy Proc 1993;14:321-326.

25. Campbell S, Hood I, Ryan D. Death as as result of asthma in Wayne County medical examiner cases, 1975-1987. J Forensic Sci 1990;35:356-364.

26. Preston HV, Bowen DAL. Asthma deaths: a review. Med Sci Law 1987;27:89-94.

27. Weiss KB. Seasonal trends in US asthma hospitalizations and mortality. JAMA 1990;263:2323-2328.

28. Jones K, Berrill WT, Bromly CL, Hendrick DJ. A confidential enquiry into certified asthma deaths in the North of England, 1994-96: influence of co-morbidity and diagnostic inaccuracy. Respir Med 1999;93:923-927.

29. Spitzer WO, Suissa S, Ernst P. The use of beta agonists and the risk of death and near death from asthma. $\mathrm{N}$ Engl $\mathrm{J}$ Med 1992:326:501-506.

30. Wilson JD, Sutherland DC, Thomas AC. Has the change to beta-agonists combined with oral theophylline increased cases of fatal asthma? Lancet 1981;1:1235-1237.

31. Kurland G, Williams J, Lewiston NJ. Fatal myocardial toxicity during continuous infusion intravenous isoproterenol therapy of asthma. J Allergy Clin Immunol 979;63: 407-411.

32. Wasserfallen J-B, Schaller M-D, Feihl F, Perret CH. Sudden asphyxic asthma: a distinct entity? Am Rev Respir Dis 1990;142:108-111.

33. Benatar SR. Fatal Asthma. N Engl J Med 1986;314(7): 423-429.

34. Macdonald JB, Seaton A, Williams DA. Asthma deaths in Cardiff 1963-74: 90 deaths outside hospital. Br Med J 1976; 1:1493-1495.

35. Messer JW, Peters GA, Bennett WA. Causes of death and pathologic findings in 304 cases of bronchial asthma. Chest 1960;38:616-624.

36. Sur S, Crotty TB, Kephart GM, et al. Sudden-onset fatal asthma. A distinct entity with few eosinophils and relatively more neutrophils in the airway submucosa? Am Rev Respir Dis 1993;148:713-719.

37. Reid LM. The presence or absence of bronchial mucus in fatal asthma. J Allergy Clin Immunol 1987;80:415-416

38. Awadh N, Muller NL, Park CS, Abboud RT, FitzGerald JM. Airway wall thickness in patients with near fatal asthma and control groups; assessment with high resolution computed tomographic scanning. Thorax 1998;53:248-253.

39. Kuwano K, Bosken CH, Pare PD, Bai TR, Wiggs BR, Hogg JC. Small airways dimensions in asthma and in chronic obstructive pulmonary disease. Am Rev Respir Dis 1993; 148:1220-1225.

40. Heard BE, Hossain S. Hyperplasia of bronchial muscle in asthma. J Pathol 1973;110:319-331.

41. Dunnill MS. The pathology of asthma, with special reference to changes in the bronchial mucosa. J Clin Pathol 1960;13:27-33.

42. Li X, Wilson JW. Increased vascularity of the bronchial mucosa in mild asthma. Am J Respir Crit Care Med 1997; 156:229-233.

43. Laitinen A, Laitinen LA. Vascular beds in the airways of normal subjects and asthmatics. Eur Respir J 1990;3: S658-S662. 
44. Carroll N, Elliot J, Morton A, James A. The structure of large and small airways in nonfatal and fatal asthma. Am Rev Respir Dis 1993;147:405-410.

45. Roche WR, Beasley R, Williams JH, Holgate ST. Subepithelial fibrosis in the bronchi of asthmatics. Lancet 1989; 1:520-524.

46. Ward C, Pais M, Bish R, et al. Airway inflammation, basement membrane thickening and bronchial hyperresponsiveness in asthma. Thorax 2002;57:309-316.

47. Ordonez CL, Khashayer R, Wong HH, et al. Mild and moderate asthma is associated with airway goblet cell hyperplasia and abnormalities in mucin gene expression. Am J Respir Crit Care Med 2001;163:517-523.

48. Groneberg DA, Eynott PR, Lim S, et al. Expression of respiratory mucins in fatal status asthmaticus and mild asthma. Histopathology 2002;40:367-373.

49. Cluroe A, Holloway L, Thomson K, Purdie G, Beasley R. Bronchial gland duct ectasis in fatal bronchial asthma: association with interstitial emphysema. J Clin Pathol 1989;42:1026-1031.

50. Dail DH, Hammer SP. Pulmonary pathology, 2nd ed. New York: Springer-Verlag, 1995:537-563.

51. Kepley, CL, McFeeley, PJ, Oliver, JM, Lipscomb, MF. Immunohistochemical detection of human basophils in postmortem cases of fatal asthma. Am J Respir Crit Care Med 2001;164(6):1053-1058.

52. Tatum AM, Greenberger PA, Mileusnic D, Donoghue ER, Lifschultz BD. Clinical, pathologic, and toxicologic findings in asthma deaths in Cook County, Illinois. Allergy Asthma Proc 2001;22(5):285-291.

53. Naylor B. The shedding of the mucosa of the bronchial tree in asthma. Thorax 1962;17:69-72.

54. Borchers AT, Naguwa SM, Keen CL, Gershwin ME. The diagnosis and management of anaphylaxis. Comp Ther 2004;30(2):111-120.

55. Kemp SF, Lockey RF. Anaphylaxis: A review of causes and mechanisms. J Allergy Clin Immunol 2002;110(3):341-348.

56. Yocum MW, Butterfield JH, Klein JS, Volcheck GW, Schroeder DR, Silverstein MD. Epidemiology of anaphylaxis in Olmsted County: a population-based study. J Allergy Clin Immunol 1999;104:452-456.

57. Kemp SF. Anaphylaxis: current concepts in pathophysiology, diagnosis, and management. Immunol Allergy Clin North Am 2001;21:611-634.

58. Edston E, van Hage-Hamstem M. Postmortem diagnosis of anaphylaxis. In: Tsokos M, ed. Forensic pathology reviews, vol 3. Totowa, NJ: Humana Press, 2005:267-281

59. Brown AFT, McKinnon D, Chu K. Emergency department anaphylaxis: a review of 142 patients in a single year. J Allergy Clin Immunol 2001;108:861-868.

60. Lieberman P. Anaphylaxis and anaphylactoid reactions. In: Middleton E Jr, Reed CE, Ellis EF, et al., eds. Allergy: principles and practice, 5th ed. St. Louis: Mosby-Year Book, 1998:1079-1092.

61. Stewart GE, Lockey RF. Systemic reactions from allergen immunotherapy. J Allergy Clin Immunol 1992;90:567578.

62. Veien M, Szlam F, Holden JT, et al. Mechanisms of nonimmunological histamine and tryptase release from human cutaneous mast cells. Anesthesiology 2000;92:1074-1081.
63. Edston E, van Hage-Hamsten M. Anaphylactoid shock: a common cause of death in heroin addicts? Allergy 1997; 52:950-954.

64. Lin RY, Schwartz LB, Curry A, et al. Histamine and tryptase levels in patients with acute allergic reactions: an emergency department-based study. J Allergy Clin Immunol 2000;106:65-71.

65. Smith PL, Kagey-Sobotka A, Bleecker ER, et al. Physiologic manifestations of human anaphylaxis. J Clin Invest 1980;66:1072-1080.

66. Lockey RF, Bukantz SC. Allergic emergencies. Med Clin North Am 1974;58:147-156.

67. Kemp SF, Lockey RF, Wolf BL, Lieberman P. Anaphylaxis: a review of 266 cases. Arch Intern Med 1995;155:1749-54.

68. Douglas DM, Sukenick E, Andrade WP, Brown JS. Biphasic systemic anaphylaxis: an inpatient and outpatient study. J Allergy Clin Immunol 1994;93:977-985.

69. Brazil E, MacNamara AF. Not so immediate hypersensitivity-the danger of biphasic allergic reactions. J Accid Emerg Med 1998;15:252-253.

70. Randall B, Butts J, Halsey JF. Elevated postmortem tryptase in the absence of anaphylaxis. J Forensic Sci 1995; 40(2):208-211.

71. Pumphrey RS, Roberts IS. Postmortem findings after fatal anaphylactic reactions. J Clin Pathol 2000;53(4):273-276.

72. Horn KD, Halsey JF, Zumwalt RE. Utilization of serum tryptase and immunoglobulin E assay in the postmortem diagnosis of anaphylaxis. Am J Forensic Med Pathol 2004; 25(1):37-43.

73. Schartz LB, Yunginger JW, Miller J, et al. Time course of appearance and disappearance of human mast cell tryptase in the circulation after anaphylaxis. J Clin Invest 1989; 83:1551-1555.

74. Edston E, van Hage-Hamsten M. beta-Tryptase measurements postmortem in anaphylactic deaths and in controls. Forensic Sci Int 1998;93:135-142.

75. Riches KJ, Gillis D, James RA. An autopsy approach to bee sting-related deaths. Pathology 2002;34:257-262.

76. Becker AB, Mactavish G, Frith E, Desiardins PRE. Postmortem stability of serum tryptase and immunoglobulin E. J Allergy Clin Immunol 1995;95:369(abstr).

77. Yunginger JW, Nelson DR, Squillace DL, et al. Laboratory investigation of deaths due to anaphylaxis. J Forensic Sci 1991;36(3):857-865.

78. Schwartz HJ, Squillace DL, Sher TH, et al. Studies in stinging insect hypersensitivity: postmortem demonstration of antivenom IgE antibody in possible sting-related sudden death. Am J Clin Pathol 1986;85:607-610.

79. Dantzker RS, Ayres SM, et al. Primary pulmonary hypertension: a national prospective study. Ann Intern Med 1987;107:216-223.

80. Simonneau G, Galie NL, Rubin J, et al. Third World Symposium on pulmonary arterial hypertension (in Venice, June 23-25, 2003). Clinical classification of pulmonary hypertension. J Am Coll Cardiol 2004;43:4s-12s.

81. Rubin LH. Primary pulmonary hypertension. N Engl J Med 1997;336:111-117.

82. Ackermann DM, Edwards WD. Sudden death as the initial manifestation of primary pulmonary hypertension. Report of four cases. Am J Forensic Med Pathol 1987;8(2):97-102. 
83. Ghamra ZW, Dweik RA. Primary pulmonary hypertension: an overview of epidemiology and pathogenesis. Cleve Clin J Med 2003;70(suppl 1):S2-8.

84. Abenhaim L, Moride Y, Brenot F, et al. Appetite-suppressant drugs and the risk of primary pulmonary hypertension. N Engl J Med 1996;335:609-616.

85. Rubin LJ, Barst RJ, Kaiser LR, et al. Primary pulmonary hypertension. Chest 1993;104:236-250.

86. Brown DL, Wetli CV, Davis JH. Sudden unexpected death from primary pulmonary hypertension. J Forensic Sci 1981;26(2):381-386.

87. Arena V, DeGiorgio F, Abbate A, Capelli A, DeMercurio D, Carbone A. Fatal pulmonary arterial dissection and sudden death as initial manifestation of primary pulmonary hypertension: a case report. Cardiovasc Pathol 2004; 13(4):230-232.

88. Consensus Conference: Prevention of venous thrombosis and pulmonary embolism. JAMA 1986;256:744-749.

89. Mortenthaler TI, Ryu JH. Clinical characteristics of fatal pulmonary embolism in a referral hospital. Mayo Clin Proc 1995;70:417-424.

90. Carson JL, Kelley MA, Duff A, et al. The clinical course of pulmonary embolism. N Engl J Med 1992;326:1240-1250.

91. Weg JG. Pulmonary embolism: diagnosis and treatment. Appl Cardiopulm Pathophysiol 1988;2:23-25.

92. Dalen JE, Alpert JS. Natural history of pulmonary embolism. Prog Cardiovasc Dis 1975;17:259-270.

93. Coon WW: Venous thromboembolism, prevalence, risk factors and prevention. Clin Chest Med 1984 3:391-401.

94. Escoffery CT, Shirley SE. Causes of sudden natural death in Jamaica: a medicolegal (coroner's) autopsy study from the University Hospital of the West Indies. Forensic Sci Int 2002;129(2):116-121.

95. Ely SF, Gill RJ. Fatal pulmonary thromboembolism and hereditary thrombophilias. J Forensic Sci 2005;50(2):411418.

96. Martinelli I. Risk factors in venous thromboembolism. Thromb Haemost 2001;86:395-403.

97. Turpie AG, Chin BS, Lip GY. Venous thromboembolism: pathophysiology, clinical features, and prevention. (Clinical review: ABC of antithrombotic therapy.) Br Med J 2002;325(7369):887-890.

98. Stein PD, Terrin ML, Hales CA, et al. Clinical laboratory roentgenographic and electrocardiographic findings in patients with acute pulmonary embolism and no preexisting cardiac or pulmonary disease. Chest 1991;100:598-603.

99. Stein PD, Saltzman HA, Weg JG. Clinical characteristics of patients with acute pulmonary embolism. Am J Cardiol 1991;68:1723-1724.

100. The PIOPED Investigators. Value of the ventilation/perfusion scan in acute pulmonary embolism: result of the prospective investigators of pulmonary embolism diagnosis (PIOPED). JAMA 1990;263:2753-2759.

101. The Urokinase Pulmonary Embolism Trial: a national cooperative study. Circulation 1973;45(suppl):1-108.

102. Urokinase-Streptokinase Pulmonary Embolism Trial: Phase 2 results. A cooperative study. JAMA 1974;229: 1606-1613.

103. Rodger M, Wells PS. Diagnosis of pulmonary embolism. Thromb Res 2001;103:225-238.
104. Anderson FA Jr, Wheeler HB. Venous thromboembolism: risk factors and prophylaxis. Clin Chest Med 1995;16: 235-251.

105. Miller EJ, Marques MB, Simons GT. Etiology of pulmonary thromboembolism in the absence of commonly recognized risk factors. Am J Forensic Med Pathol 2003;24: 329-333.

106. Seligsohn U, Lubetsky A. Genetic susceptibility to venous thrombosis. N Engl J Med 2001;344:1222-1231.

107. Andrew TA, Fairweather R. Prothrombin G20210A mutation and sudden death (case reports). Am J Forensic Med Pathol 2003;24(4):377-380.

108. Price DT, Ridker PM, Factor V. Leiden mutation and the risks for thromboembolic disease: A clinical perspective. Ann Intern Med 1997;127:895-903.

109. Nicolaides AN, O'Connell JD. Origin and distribution of thrombi in patients presenting with clinical deep venous thrombosis. In: Nicolaides AN, ed. Thromboembolism etiology: advances in prevention and management. Baltimore: University Park Press, 1975:177-180.

110. Fowler EF, Bollinger JA. Pulmonary embolism. Surgery 1954;36:650-663.

111. Edlow JA. Emergency department management of pulmonary embolism. Emerg Med Clin North Am 2001;19: 995-1011.

112. Stein PD, Henry JW. Clinical characteristics of patients with acute pulmonary embolism stratified according to their presenting syndromes. Chest 1997;112:974-979.

113. Sadosty AT, Boie ET, Stead LG. Pulmonary embolism. Emerg Med Clin North Am 2003;21:363-384.

114. Rippe JM, Irwin RS, Fink MP, Cerra FB. Intensive care medicine, 3rd ed. Boston: Little, Brown, 1996.

115. Presti B, Berthrong M, Sherwin RM. Chronic thrombosis of major pulmonary arteries. Hum Pathol 1990;21(6): 601-606.

116. Stebbens WE, Lie JT. Vascular pathology. London: Chapman \& Hall Medical, 1995:501.

117. Dunnill MS. Pulmonary embolism. Br J Surg 1968;55(10): 790-794.

118. Ro A, Kageyama N, Tanifuji T, et al. Histopathological study of pulmonary arteries in 14 autopsy cases with massive pulmonary thromboembolism. Legal Med 2003; 5(suppl 1):S315-317.

119. Zenker FA. Beitrage zur normalen und pathologischen anatomic der lunge. Dresden: J Braunsdorf, 1862;31

120. Chan KM, Tham KT, Chiu HS, Chow YN, Leung PC. Post-traumatic fat embolism-its clinical and subclinical presentations. J Trauma 1984;24:45-49.

121. Glas WW, Musselmann Davis HL. The source of fat in fat embolism. Arch Surg 1955;71:600-604.

122. Mudd KL, Hunt A, Matherly RC, et al. Analysis of pulmonary fat embolism in blunt force fatalities. J Trauma 2000; 48(4):711-715.

123. Levy D. The fat embolism syndrome. Clin Orthop 1990; 261:281-286.

124. ten Duis HJ. The fat embolism syndrome. Injury 1997;28(2): 77-85.

125. Dudney TM, Elliott CG. Pulmonary embolism from amniotic fluid, fat and air. Prog Cardiovasc Dis 1994;36: 447-474. 
126. Sevitt S. Fat embolism. London: Butterworth, 1962.

127. Baltensweiler J. Fettemboliesyndrom, Klinik und Prophylaxe. Bern, Stuttgart, Wein: Hans Humber Verlag, 1977.

128. Beck JP, Collins JA. Theoretical and clinical aspects of posttraumatic fat embolism syndrome. In: Calandruccio RA ed. The American Academy of Orthopedic Surgeons. Instr Course Lect. Mosby, St. Louis. MO. 1973;22:38-87.

129. Herndon JH. The syndrome of fat embolism. South Med J 1975;68:1577-1584.

130. Sevitt S. Pathophysiology of systemic embolism - the brain. In: Sevitt S, ed. Fat embolism. London: Butterworth, 1962: 143.

131. Gurd AR, Wilson RI. The fat embolism syndrome. J Bone Joint Surg (Br) 1974;56:408-416.

132. Peltier LF, Collins JA, Evart CM, et al. Fat embolism. Arch Surg 1974;109:12-16.

133. Ross APJ. The fat embolism syndrome: with special reference to the importance of hypoxia in the syndrome. Ann R Coll Surg Engl 1970;46:159-171.

134. Jacobson DM, Terrence CF, Reinmuth OM. The neurological manifestations of fat embolism. Neurology 1986;36: 847-851.

135. Peltier LF. The diagnosis of fat embolism. Surg Gynecol Obstet 1965;121:630-635.

136. Evarts CM. The fat embolism syndrome: a review. Surg Clin North Am 1970;50:493-507.

137. Bhasrakan CS. Bone marrow embolism in traumatic death. Pathol Microbiol 1969;34:105-111.

138. Weisz GM. Fat embolism. Curr Probl Surg 1974;1-54.

139. Weinberg H, Finsterbush A. Fat embolism: vascular damage to bone due to blunt trauma. Clin Orthop 1972;83: 273-285.

140. Hiss J, Kahana T, Kugel C. Beaten to death: why do they die? J Trauma 1996;40:27-30.

141. Kerstell J. Pathogenesis of post-traumatic fat embolism. Am J Surg 1971;121:712-715.

142. Adar R. Pathogenesis and treatment of fat embolism. Harefuah J Isr Med Assoc 1972;83:491-493.

143. Deland FH. Bone marrow embolism and associated fat embolism to the lungs. Thesis, Graduate School, University of Minnesota, 1956.

144. James ES. Fat embolism. Can Med Assoc J 1959;62:548550.

145. Peltier LF. An appraisal of the problem of fat embolism. Int Abst Surg 1957;104:313.

146. Lehman, EP, Moore, RM. Fat embolism: Including experimental production without trauma. Arch Surg 1927;14: 621-662.

147. Tracy RE, Walia P. A method to fix lipids for staining fat embolism in paraffin sections. Histopathology 2002;41: 75-79.

148. Chastre J, Fagon JY, Soler P, et al. Bronchoalveolar lavage for rapid diagnosis of the fat embolism syndrome in trauma patients. Ann Intern Med 1990;113:583-588.

149. Masson RG, Ruggieri J, Siddiqui MM. Pulmonary microvascular cytology. A new diagnosis application of the pulmonary artery catheter. Chest 1985;88:908-914.

150. Castella X, Valles J, Cabezuelo MA, et al. Fat embolism syndrome and pulmonary microvascular cytology. Chest 1992;101:1710-1711.
151. Bannier B, Poirer T, Viaud JY, et al. Fat embolism diagnostic interest of the bronchoalveolar lavage. Intensive Care Med 1992;18:59-60.

152. Francis TJ, Dutka, AJ, Hallenbeck JM. Pathophysiology of decompression sickness. In: Bove AA, Davis JC, eds. Diving medicine. Philadelphia: Saunders, 1990:170-187.

153. Orebaugh SL. Venous air embolism: clinical and experimental considerations. Crit Care Med 1992;20(8):11691177.

154. Borja A. Current status of intraclavicular subclavian vein catheterization. Ann Thorac Surg 1972;13:615-622.

155. Matjasko J, Petrozza P, Cohen M, et al. Anesthesia and surgery in the seated position: analysis of 554 cases. Neurosurgery 1985;17:695-702.

156. Standefer M, Bay JW, Trusso R. The sitting position in neurosurgery: a retrospective analysis of 488 cases. Neurosurgery 1984;14:649-658.

157. Bray P, Myers RAM, Cowley, RA. Orogenital sex as a cause of nonfatal air embolism in pregnancy. Obstet Gynecol 1983; 61:653-657.

158. Martland HS. Air embolism: fatal air embolism due to powder insufflators used in gynecological treatments. Am J Surg 1945;68:194-169.

159. Yeakel AE. Lethal air embolism from plastic blood-storage container. JAMA 1968;204:267-269.

160. Flanagan JP, Gardisar IA, Gross RJ, Kelly TR. Air embolus: a lethal complication of subclavian venipuncture. $\mathrm{N}$ Engl J Med 1969;281:488-489.

161. Toung TJK, Rossberg RI, Hutchins GM. Volume of air in a lethal venous air embolism (case reports). Anesthesiology 2001;94(2):360-361.

162. Hollingsworth HM, Irwin RS. Acute respiratory failure in pregnancy. Clin Chest Med 1992;13(4):723-740.

163. Kashuk JL, Penn I. Air embolism after central venous catheterization. Surg Gynecol Obstet 1984;159:249-252.

164. Finkbeiner WE, Ursell PC, Davis RL. Autopsy pathology: a manual and atlas. Philadelphia: Churchill Livingstone, 2004:86-90.

165. Baker RD. Postmortem examination: specific methods and procedures. Philadelphia: WB Saunders, 1967.

166. Kulka W. A practical device for demonstrating air embolism. Arch Pathol 1949;48:366-369.

167. Van Ieperen L. Venous air embolism as a cause of deatha method of investigation. S Afr Med J 1983;63:442-443.

168. Bajanowski T, West W, Brinkmann B. Proof of fatal air embolism. Int J Legal Med 1998;111:208-211.

169. Buchanan D, Mason JK. Occurrence of pulmonary fat and bone marrow embolism. Am J Forensic Med Pathol 1982; 3(1):73-78.

170. Schinella RA. Bone marrow emboli. Arch Pathol 1973;95: 386-391.

171. Tozzini S, Anichini C, Gori F, Bartolini G. Pulmonary embolism by liver tissue. Am J Forensic Med Pathol 2004; 25(1):87

172. Michalodimitrakis M, Tsatsakis A. Massive pulmonary embolism by liver tissue. Med Sci Law 1998;38:85-87.

173. Walz MV, Walz MK. Pulmonary embolism caused by liver tissue. Aktuelle Traumatol 1992;22:102-103.

174. Voitk AJ, Munkittrich RC.A fatal pulmonary embolus composed of gross liver tissue. Can J Surg 1986;29:218-219. 
175. Moar JJ. Pulmonary embolism due to hepatic tissue: a forensic vital sign? A case report. S Afr Med J 1984;66:699-700.

176. Chan GS, Ng WK, Ng IO, Dickens P. Sudden death from massive pulmonary tumor embolism due to hepatocellular carcinoma. Forensic Sci Int 2000;108(3):215-221.

177. Sherwood SF, Hartsock RL. Thoracic injuries. In: McQuillian KA, Von Rueden KT, Hartstock RL, Flynn $\mathrm{MB}$, Whalen E, eds. Trauma nursing from resuscitation through rehabilitation, 3rd ed. Philadelphia: Saunders, 2002:543-590.

178. Jantz MA, Pierson DJ. Pneumothorax and barotraumas. Clin Chest Med 1994;15:75-91.

179. Putukian M. Pneumothorax and pneumomediastinum. Clin Sports Med 2004;23:443-454.

180. Marnejon T, Sarac S, Cropp AJ. Spontaneous pneumothorax in weightlifters. J Sports Med Phys Fitness 1995;35: 124-126.

181. Simons S. Pneumothorax and exercise. Sports Med Consult 2000;1:1-6.

182. Boschat J, Blanc JJ, Clavier J, Kerbrat G, N'guyen-Huu. Spontaneous pneumothorax in the adult. Problems of its development and treatment. Poumon Coeur 1976;32:43-46 (in French).

183. Sawyer MAJ. Blunt chest trauma. EMed J (serial online) 2004. http://www.emedicine.com/med/topic3658.htm.

184. Volk CP, McFarland EG, Horsmon G. Pneumothorax: on field recognition. Phys Sportsmed 1995;23:43-46.

185. Yamamoto L, Schroeder C, Morley D, Beliveau C. Thoracic trauma: the deadly dozen (major trauma). Crit Care Nursing Q 2005;28(1):22-40.

186. Ludwig J, Miller WE, Sessler AD. Clinically unsuspected pneumothorax: a postmortem roentgenographic study. Arch Pathol 1970;90:274-277.

187. Box CR. Post-mortem technique. London: J\&A Churchill, 1910.

188. Pralow JA, Barnard JJ. Pregnancy-related maternal deaths. Am J Forensic Med Pathol 2004;25(3):220-236.

189. Grimes DA. The morbidity and mortality of pregnancy: Still risky business. Am J Obstet Gynecol 1994;170: 1489-1494.

190. Chatelain SM, Quirk G. Amniotic and thromboembolism. Clin Obstet Gynecol 1990;33(3):473-481.

191. Stone SE, Morris TA. Pulmonary embolism and pregnancy. Crit Care Clin 2004;20:661-677.

192. Gherman RB, Goodwin TM, Leung B, Byrne JD, Hethumumi R, Montoro M. Incidence, clinical characteristics, and timing of objectively diagnosed venous thromboembolism during pregnancy. Obstet Gynecol 1999;94(5 pt 1): 730-734.

193. Toglia MR, Weg JG. Venous thromboembolism during pregnancy. N Engl J Med 1996;335(2):108-114.

194. Clark P, Brennand J, Conkie JA, McCall F, Greer IA, Walker ID. Activated protein $\mathrm{C}$ sensitivity, protein C, protein $\mathrm{S}$ and coagulation in normal pregnancy. Thromb Haemost 1998;79(6):1166-1170.

195. Brown HL, Hiett AK. Deep venous thrombosis and pulmonary embolism. Clin Obstet Gynecol 1996;39:87-100.

196. Atrash HK, Koonin LM, Lawson HW, et al. Maternal mortality in the United States, 1979-1986. Obstet Gynecol 1990;76:1055-1060.
197. Rutherford SE, Phelan JP. Thromboembolic disease in pregnancy. Clin Perinatol 1986;13:719-739.

198. Rutherford SE, Phelan JP. Deep venous thrombosis and pulmonary embolism in pregnancy. Obstet Gynecol Clin North Am 1991;18(2):345-370.

199. Kakkar VV, Sasahara AA. Diagnosis of venous thrombosis and pulmonary embolism. In: Bloom AL, Thomas DP, eds. Haemostasis and thrombosis. Edinburgh: Churchill Livingstone, 1987:779.

200. Weiner CP. Diagnosis and management of thromboembolic disease during pregnancy. Clin Obstet Gynecol 1985; 28:107-118.

201. Dawkins KD, Burke CM, Billingham ME, Jamieson SW. Primary pulmonary hypertension and pregnancy. Chest 1986;89:383-388.

202. Kiss H, Egarter C, Asseryanis E, et al. Primary pulmonary hypertension in pregnancy: a case report. Am J Obstet Gynecol 1995;172:1052-1054.

203. Feijen HWH, Hein PR, van Lakwijk-Vondrovicova EL, Nijhuis GMM. Primary pulmonary hypertension and pregnancy. Eur J Obstet Gynecol Reprod Biol 1983;15:159164.

204. McCaffrey RM, Dunn LJ. Primary pulmonary hypertension in pregnancy. Obstet Gynecol Surv 1964;19:567-591.

205. Roberts NV, Keast PJ. Pulmonary hypertension and pregnancy-a lethal combination. Anaesth Intensive Care 1990;18:366-374.

206. Takeuchi T, Nishii O, Okamura T, Yaginuma T. Primary pulmonary hypertension in pregnancy. Int $\mathrm{J}$ Gynecol Obstet 1988;26:145-150.

207. Meyer JR. Embolia pulmonary amnio-caseosa. Braz J Med Biol Res 1926;1:301-303.

208. Steiner PE, Lushbaugh CC. Landmark Article. Maternal pulmonary embolism by amniotic fluid as a cause of obstetric shock and unexpected deaths in obstetrics. JAMA 1986;255:2187-2203.

209. Tuffnell DJ, Johnson H. Amniotic fluid embolism: the UK register. Hosp Med 2000;61(8):532-534.

210. Morgan M. Amniotic fluid embolism. Anaesthesia 1979; 34:20-32.

211. Prahlow JA, Lantz PE. Amniotic fluid embolism with paradoxical embolization. Am Soc Clin Pathol Check Sample Program. Forensic Pathol 1993;35:1-4.

212. Tuffnell DJ. Amniotic fluid embolism. Curr Opin Obstet Gynecol 2003;15(2):119-122.

213. Burrows A, Khoo,SK. The amniotic fluid embolism syndrome: 10 years' experience at a major teaching hospital. Aust NZ J Obstet Gynaecol 1995;35:245-250.

214. Michigan State Medical Society. Maternal Mortality Study, 1960-1964.

215. Philip RS. Amniotic fluid embolism. N Y J Med 1967;67: 2085-2088.

216. Clark SL, Hankins GDV, Dudley DA, Dildy GA, Porter TF. Amniotic fluid embolism: analysis of the national registry. Am J Obstet Gynecol 1995;172:1158-1169.

217. Gilbert WM, Danielsen B. Amniotic fluid embolism: decreased mortality in a population-based study. Obstet Gynecol 1999;93(6):973-977.

218. Tuffnell DJ. Amniotic fluid embolism. Maternal mortality and morbidity. In: Proceedings of the 43rd Royal College 
of Obstetricians and Gynaecologists Study Group. London: Royal College of Obstetricians and Gynaecologists Press, 2002.

219. Locksmith GJ. Amniotic fluid embolism. Obst Gynecol North Am 1999;26(3):435-444.

220. Davies S. Obstetrical and pediatric anesthesia. Amniotic fluid embolus: a review of the literature. Can J Anesth 2001;48(1):88-98.

221. Aurangzeb I, George L, Raoof S. Amniotic fluid embolism. Crit Care Clin 2004;20:643-650.

222. Clark SL. Amniotic fluid embolism. Crit Care Clin 1991; 7:877-882.

223. Masson RG. Amniotic fluid embolism. Clin Chest Med 1992;13:657-665.

224. McDougall RJ, Duke GJ. Amniotic fluid embolism syndrome: case report and review. Anaesth Intensive Care 1995;23:735-740.

225. Meier PR, Bowes WA Jr. Amniotic fluid embolus-like syndrome presenting in the second trimester of pregnancy. Obstet Gynecol 1983;61:31s-34s.

226. Clark SL. New concepts in amniotic fluid embolism: a review. Obstet Gynecol Surv 1990;45:360-368.

227. Nishio H, Matsui K, Miyazaki T, et al. A fatal case of amniotic fluid embolism with elevation of serum mast cell tryptase. Forensic Sci Int 2002;126:53-56.

228. Farrar SC, Gherman RB. Serum tryptase in a woman with amniotic fluid embolism: a case report. J Reprod Med 2001;46:926-928.

229. Dorne R, Pommier C, Emery JC, et al. Embolie de liquide amniotique: evolution favorable après embolisation therapeutique des arteres uterines. Ann Fr Anesth Reanim 2002;21:431-435.

230. Benson MD, Kobayashi H, Silver RK, et al. Immunologic studies in presumed amniotic fluid embolism. Obstet Gynecol 2001;97:510-514.

231. Clark SL, Montz FJ, Phelan JP. Hemodynamic alterations association with amniotic fluid embolism: a reappraisal. Am J Obstet Gynecol 1985;151(5):617-621.

232. Attwood HD. Amniotic fluid embolism. In: Sommers SC, ed. Pathol Annu 1972;7:145-172.

233. Kobayashi $\mathrm{H}$, Ooi H, Hayakawa $\mathrm{H}$, et al. Histological diagnosis of amniotic fluid embolism by monoclonal antibody TKH-2 that recognizes NeuAc alpha 2-6GalNAc epitope. Hum Pathol 1997;28(4):428-433.

234. Wasser WG, Tessler S, Kamath C, Sackin AJ. Nonfatal amniotic fluid embolism: a case report of post-partum respiratory distress with histopathologic studies. Mt Sinai J Med 1979;46(4):388-391.

235. Stromme WB, Fromke VL. Amniotic fluid embolism and disseminated intravascular coagulation after evacuation of a missed abortion. Obstet Gynecol 1978;52:76s-80s.

236. Dolyniuk M, Orfei E, Vania H, et al. Rapid diagnosis of amniotic fluid embolism. Obstet Gynecol 1983;61:28s-30s.

237. Lee W, Ginsburg KA, Cotton DB, Kaufman RH. Squamous and trophoblastic cells in the maternal pulmonary circulation identified by invasive hemodynamic monitoring during the peripartum period. Am J Obstet Gynecol 1986:155:999-1001.

238. Attwood HD, Park WW. Embolism to the lungs by trophoblast. J Obstet Gynecol 1961;68:611-617.
239. Batman PA, Thomlinson J, Moore VC, et al. Death due to air embolism during sexual intercourse in the puerperium. Postgrad Med J 1998;74:612-613.

240. Bray P, Myers RAM, Cowley RA. Orogenital sex as a cause of nonfatal air embolism in pregnancy. Obstet Gynecol 1983;61:653-657.

241. Fatteh A, Leach WB, Wilkinson CA. Fatal air embolism in pregnancy resulting from orogenital sex play. Forensic Sci 1973;2:247-250.

242. Fyke FE III, Kazmier FJ, Harms RW. Venous air embolism: life-threatening complication of orogenital sex during pregnancy. Am J Med 1985;78:333-336.

243. Kwon HL, Belanger K, Bracken MB. Asthma prevalence among pregnant and childbearing aged women in the United States: estimates from national health surveys. Ann Epidemiol 2003;13(5):317-324.

244. Venkataraman MT, Shanies HM. Pregnancy and asthma. J Asthma 1997;34:265-271.

245. White RJ, Coutts II, Gibbs CJ, MacIntyre C. A prospective study of asthma during pregnancy and the puerperium. Respir Med 1989;83(2):103-106.

246. Turner ES, Greenberger PA, Paterson R. Management of the pregnant asthmatic. Ann Intern Med 1980;103:563573.

247. Kircher S, Schatz M, Long L. Variables affecting asthma course during pregnancy. Ann Allergy Asthma Immunol 2002;89(5):463-465.

248. Stenius-Aarniala B, Piirila P, Teramo K. Asthma and pregnancy: a prospective study of 198 pregnancies. Thorax 1988;43(1):12-18.

249. Schatz M, Harden K, Forsythe A, et al. The course of asthma of during pregnancy, postpartum and with successive pregnancies: a prospective analysis. J Allergy Clin Immunol 1988;81:509-517.

250. Guy ES, Kirumaki A, Hanania NA. Acute asthma in pregnancy. Crit Care Clin 2004;20:731-745.

251. Cohle SD, Petty CS. Sudden death caused by embolization of trophoblast from hydatidiform mole. J Forensic Sci 1985;30:1279-1283.

252. Kingston NJ, Baillie T, Chan YF, Reddy DJ, Stables SR. Pulmonary embolization by chorionic villi Causing maternal death after a car crash. Am J Forensic Med Pathol 2003;24:193-197.

253. Saukko P, Knight B. Knight's forensic pathology, 3rd ed. London: Arnold, 2004.

254. Adelson L. The pathology of homicide. Springfield, IL: Charles C. Thomas, 1974.

255. Centers for Disease Control. Guidelines for death scene investigation of sudden, unexpected infant deaths: Recommendations of the interagency panel on sudden infant death syndrome. MMWR 1996;45(RR-10):1-22.

256. Beckwith JB. Defining the sudden infant death syndrome. Arch Pediatr Adolesc Med 2003;157:286-290.

257. Byard RW. Sudden infant death syndrome. In Byard RW, Cohle SD, eds. Sudden death in infancy, childhood and adolescence. New York: Cambridge University Press, 1994: 417-497.

258. Goldberg J, Hornung R, Yamashita T, et al. Age at death and risk factors in sudden infant death syndrome. Aust Paediatr J 1986;22(suppl):21-28. 
259. Ford KM, Linker LA. Compliance of licensed child-care centers with the American Academy of Pediatrics' recommendation for infant sleep positions. J Comm Health Nurs 2002;19:83-91.

260. Campbell Daley K. Update on sudden infant death syndrome. Curr Opin Pediatr 2004;16(2):227-232.

261. Naeye RL. Pulmonary arterial abnormalities in the sudden infant death syndrome. N Engl J Med 1973;289:11671170.

262. Naeye RL. Sudden infant death. Sci Am 1980;242:56-62.

263. Valdes-Dapena M. The morphology of the sudden infant death syndrome-an update, 1984. In: Harper RM, Hoffman HJ, et al., eds. Sudden infant death syndrome: risk factors and basic mechanisms. New York: PMA, 1987:143.

264. Valdes-Dapena M. Sudden infant death syndrome: Morphology update for forensic pathologists-1985. Forensic Sci Int 1986;30:177-186.

265. Beckwith JB. Chronic hypoxia in the sudden infant death syndrome: a critical review of the data base. In: Tildon JJ, Roeder LM, Steinschneider A, eds. Sudden infant death syndrome. Proceedings of the 1982 International Research Conference on the Sudden Infant Death Syndrome. New York: Academic Press, 1983:144.

266. Singer DB, Tilley E. Pulmonary arteries and arterioles normal in the sudden infant death syndrome. In: Harper RM, Hoffman HJ, eds. Sudden infant death syndrome: risk factors and basic mechanisms. New York: PMA, 1987:101.

267. Kinney HC, Burger PC, Harrell FE, Hudson RP. Reactive gliosis in the medulla oblongata of victims of sudden infant death syndrome. Pediatrics 1983;72:181-187.

268. Kinney HC, Filiano JJ. Brainstem research in sudden infant death syndrome. Pediatrician 1988;15:240-250.

269. Cote A, Gerea T, Brouillette RT, Laplante S. Circumstances leading to a change to prone sleeping in sudden infant death syndrome victims. Pediatrics 2000;106:E86.

270. Hauck FR, Herman SM, Donovan M, et al. Sleep environment and the risk of sudden infant death syndrome in an urban population: the Chicago Infant Mortality Study. Pediatrics 2003 111(5 pt 2):1207-1214.

271. Kemp J, Kowalski R, Burch P, et al. Unintentional suffocation by rebreathing: A death scene and physiologic investigation of a possible cause of sudden infant death. J Pediatr 1993;122:874-880.

272. Kinney HC, Filiano JJ, Sleeper LA et al. Decreased muscarinic receptor binding in the arcuate nucleus in sudden infant death syndrome. Science 1995;269:1446-1450.

273. Kahn A, Groswasser J, Franco P, et al. Sudden infant deaths: stress, arousal and SIDS. Early Hum Dev 2003; 75(suppl):S147-166.

274. Horne RS, Parslow PM, Harding R. Respiratory control and arousal in sleeping infants. Paediatr Respir Rev 2004;5(3):190-198.

275. AAP task force on infant positioning and SIDS: positioning and SIDS. Pediatrics 1992;89:1120-1126.

276. Malloy MH, Freeman DH. Age at death, season, and day of death as indicators of the effect of the back to sleep program on sudden infant death syndrome in the United States, 1992-1999. Arch Pediatr Adolesc Med 2004;158(4): 359-365.
277. Malloy MH, MacDorman M. Changes in the classification of sudden unexpected infant deaths: USA, 1992-2001. Pediatrics 2005;115:1247-1253.

278. Beckwith JB. Sudden infant death syndrome. Curr Probl Pediatr 1973;3:1-36.

279. Fearn S. Sudden and unexplained death of children. Lancet 1834;1:246-248.

280. Krous HF, Jordan J. The microscopic distribution of intrathoracic petechiae in sudden infant death syndrome. Arch Pathol Lab Med 1984;108:77-79.

281. Krous HF, Nadeau JM, Silva PD, Blackbourne BD. A comparison of respiratory symptoms and inflammation in sudden infant death syndrome and in accidental or inflicted infant death. Am J Forensic Med Pathol 2003; 24(1):1-8.

282. Bamford FN, MacFayden UM, Meadow SR, et al. Investigation. In: Evaluation of suspected imposed upper airway obstruction: report of a working party. London: Royal Society of Medicine Press, 1994.

283. Wanek S, Mayberry, JC. Blunt thoracic trauma: flail chest, pulmonary contusion, and blast injury. Crit Care Clin 2004; 20:71-81.

284. Injury Facts. National Safety Council; 2002.

285. Newman RJ, Jones IS. A prospective study of 413 consecutive car occupants with chest injuries. J Trauma 1984;24(2): 129-35.

286. Shorr RM, Crittenden M, Indeck M, Hartunian SL, Rodriguez A. Blunt thoracic trauma. Analysis of 515 patients. Ann Surg 1987;206(2):200-205.

287. LoCicero J III, Mattox KL. Epidemiology of chest trauma. Surg Clin North Am 1989;69(1):15-19.

288. Zuidemia GD, Rutherford RB, Ballinger WF II. The management of trauma. Philadelphia: WB Saunders, 1985.

289. Carrero R, Wayne, M. Chest trauma emergency. Med Clin North Am 1989;7:389-418.

290. Rippe JM, Irwin RS, Fink MP, Cerra FB. Intensive care medicine, 3rd ed. Philadelphia: Lippincott Williams \& Wilkins, 1995.

291. Hubble MW, Hubble JP. Principles of advanced trauma care. Albany, NY: Delmar, 2002.

292. Clark CG, Schecter WP, Trunkey DD. Variables affecting outcome in blunt chest trauma: flail chest vs. pulmonary contusion. J Trauma 1988;28(3):298-304.

293. Stevens E, Templeton AW. Traumatic nonpenetrating lung contusion. Radiology 1965;85:247-252.

294. Wiot JF. The radiologic manifestations of blunt chest trauma. JAMA 1975;231:500-503.

295. Fraser RS, Pare JAP, Fraser RG, Pare PD. Synopsis of disease of the chest, 2nd ed. Philadelphia: WB Saunders, 1979:1572-1577.

296. Johnson JM, Steinberg SR. Diaphragmatic hernias, acquired. Emed: http:/www.emeducube/, cin/med/ topics3487.htm.

297. Holm A, Bessy PQ, Aldrete JS. Diaphragmatic rupture due to blunt trauma: morbidity and mortality in 42 cases. South Med J 1988;81:956-962.

298. Bernatz PE, Burnside AF Jr, Clagett OT. Problem of the ruptured diaphragm. JAMA 1958;168:877-881.

299. Mosesso VJ. Penetrating chest trauma. In: Ferrera PC, Colucciello SA, Marx JA, Verdile VP, Gibbs MA, eds. 
Trauma management: an emergency medicine approach. St. Louis: Mosby, 2001:259-278.

300. Brooks JW. Blunt traumatic rupture of the diaphragm. Ann Thorac Surg 1978;26:199-203.

301. Bayley EW, Turcke SA. A comprehensive curriculum for trauma nursing. Boston: Jones \& Bartlett, 1992.

302. Collins JP, Ketharanathan V, McConchie I. Rupture of major bronchi resulting from closed chest injuries. Thorax 1973;28:371-375.

303. Mulder DS, Barkin JS. Injury of the trachea, bronchus, and esophagus. Trauma 1991;2:343-355.

304. Cooper GJ, Maynard RL, Cross NL, Hill JF. Casualties from terrorist bombings. J Trauma 1983;23(11):955-967.

305. Mayorga MA. The pathology of primary blast overpressure injury. Toxicology 1997;121:17-28.

306. DePalma RG, Burris DG, Champion HR, Hodgson MJ. Current concepts: blast injuries. N Engl J Med 2005;353: 1335-1342.

307. Covello VT, Peters RG, Wojtecki JG, Hyde RC. Risk communication, the West Nile virus epidemic, and bioterrorism: responding to the communication challenges posed by the intentional or unintentional release of a pathogen in an urban setting. J Urban Health 2001;78:382-391.

308. Brown RF, Cooper GJ, Maynard RL. The ultrastructure of rat lung following acute primary blast injury. Int $\mathbf{J}$ Exp Pathol 1993;74:151-162.

309. Jensen HJ, Bonding P. Experimental pressure induced rupture of the tympanic membrane in man. Acta Otolaryngol 1993;113:62-67.

310. Kratz E, Ofek B, Adler J, Abramowitz HB, Krausz MM. Primary blast injury after a bomb explosion in a civilian bus. Ann Surg 1989;209:484-448.

311. Tsokos M, Paulsen F, Petri S, Madea B, Puschel K, Turk E. Histologic, immunohistochemical, and ultrastructural findings in human blast lung injury. Am J Res Crit Care Med 2003; 168:549-555.

312. Elsayed NM. Toxicology of blast overpressure. Toxicology 1997;121:1-15.

313. Gorbunov NV, Elsayed NM, Kisin ER, Kozlov AV, Kagan VE. Air blast-induced pulmonary oxidative stress: interplay among hemoglobin, antioxidants, and lipid peroxidation. Am J Physiol 1997;272:L320-L334.

314. Elsayed NM, Armstrong KL, William MT, Cooper MF. Antioxidant loading reduces oxidative stress induced by high-energy impulse noise (blast) exposure. Toxicology 2000;155:91-99.

315. Elsayed NM, Gorbunov NV, Kagan VE. A proposed biochemical mechanism involving hemoglobin for blast overpressure-induced injury. Toxicology 1997;121:81-90.

316. Szokol JW, Alspach D, Mehta MK, Parilla BV, Liptay MJ. Intermittent airway obstruction and superior vena cava syndrome in a patient with an undiagnosed mediastinal mass after cesarean delivery. Anesth Analg 2003;97(3): 883-884.

317. Cohle SD, Trestrail JD, Graham MA, Oxley DW, Walp B, Jachimczyk J. Fatal pepper aspiration. Am J Dis Child 1998;142:633-636.

318. Sheahan K, Page DV, Kemper T, Suarez R. Childhood sudden death secondary to accidental aspiration of black pepper. Am J Forensic Med Pathol 1988;9(1):51-53.
319. Flintoff WM, Poushter DL. Aspiration of black pepper. Arch Otolaryngol Head Neck Surg 1974;100:375-376.

320. Cohle SD. Homicidal asphyxia by pepper aspiration. J Forensic Sci 1986;17:1475-1478.

321. Adelson L. Homicide by pepper. J Forensic Sci 1964:9(3); 391-395.

322. Wynne JW, Modell JH. Respiratory aspiration of stomach contents. Ann Intern Med 1977;87:466-474.

323. Roberts RB, Shirley MA. Reducing the risk of acid aspiration during cesarean section. Anesth Analg 1974;53: 859-868.

324. Davidson JT, Rubin S, Eyal Z, Polliack A. A comparison of the pulmonary response to the endotracheal instillation of $0.1 \mathrm{~N}$ hydrochloric acid and Hartmann's solution in the rabbit. Br J Anaesth 1974;46:127-132.

325. Harbord RP, Homi J. Microscopic changes in living lung after fluid aspiration. Anesth Analg (Cleve) 1970;49: 835-843.

326. Alexander IGS. The ultrastructure of the pulmonary alveolar vessels in Mendelson's (acid pulmonary aspiration) syndrome. Br J Anaesth 1968;40:408-414.

327. Idris AH, Berg RA, Bierens J, et al. Recommended guidelines for uniform reporting of data from drowning: the "Utstein style." Resuscitation 2003;108:2565-2574.

328. Baker SP, O'Neill B, Karpf RD. The injury fact book. Lexington, MA. Lexington Books. 1984.

329. Centers for Disease Control. Drownings in a private lake-North Carolina, 1981-1990. MMWR 1992;41(19): 329.

330. Anonymous. Child drowning and near drownings associated with swimming pools, Maricopa County, Arizona, 1988-1989. JAMA 1990;264(6):680.

331. Mebane GY. Drowning in a sea of tears. Alert Diver 1993;4:8.

332. Jensen LR. Submersion injuries in children younger than 5 yrs in urban Utah. West J Med 1992;157(6):641-644.

333. Spyker DA. Submersion injury: epidemiology, prevention and management. Pediatr Clin North Am 1985;32:113-135.

334. Modell JH, Bellefleur M, Davis JH. Drowning without aspiration: is this an appropriate diagnosis? J Forensic Sci 1999;44(6):1119-1123.

335. Newman AB. Submersion incidents. In: Auerbach P, ed. Wilderness medicine, 3rd ed. St. Louis: Mosby, 1995: 1209-1233.

336. Modell JH, Gaub, M, Moya, F. Physiological effects of near drowning with chlorinated fresh water, distilled water and isotonic saline. Anesthesiology 1966;27:33-41.

337. Modell JH, Moya F. Effects of volume of aspirated fluid during chlorinated fresh water drowning. Anesthesiology 1966;27:662-672.

338. Noble CS, Sharpe N. Drowning: its mechanism and treatment. Can Med Assoc J 1963;89:402-405.

339. Modell JH, Moya F, et al. Changes in blood gases and AaDO 2 during near-drowning. Anesthesiology 1968;29: 456-465.

340. Orlowski JP, Szpilman D. Drowning: rescue, resuscitation and reanimation. Pediatr Clin North Am 2001;48:627-646.

341. Modell JH, Gaub M, et al. Physiologic effects of near drowning with chlorinated fresh water, distilled water and isotonic saline. Anesthesiology 1966;27:33-41. 
342. Giammona ST, Modell JH. Drowning by total immersion: effects on pulmonary surfactant of distilled water, isotonic saline and sea water. Am J Dis Child 1967;114:612-616.

343. Orlowski JP, Abulleil MM, Phillips JM. Effects of tonicities of saline solutions on pulmonary injury in drowning. Crit Care Med 1987;15;126-130.

344. Lunetta P, Modell JH. Macroscopical, microscopical, and laboratory findings in drowning victims. In: Tsokos, M, ed. Forensic pathology reviews, vol 3. Totowa, NJ: Humana Press, 2005:3-77.

345. Swann HG. Resuscitation in semi-drowning. In: Whittenberger JL, Artificial respiration: therapy and application. Harper \& Row, New York, 1962:202-224.

346. Cot C. Les asphyxies accidentelles (submersion, electrocution, intoxication oxycarbonique) etude clinique, therapeautique et preventive.Paris, Maloine:Editions Medicales, 1931.

347. Moritz AR. Chemical methods for the determination of death by drowning. Phys Rev 1944;24:70-88.

348. Giammona ST. Drowning: pathophysiology and management. Curr Probl Pediatr 1971;3:3.

349. Modell JH. Pathophysiology and treatment of drowning and near drowning. Springfield, Ill, Thomas. 1971.

350. Knight B. Immersion deaths. In: Knight B, ed. Forensic pathology. London: Edward Arnolds, 1991.

351. Lunetta P, Modell J, Sajantila A. What is the incidence and significance of "dry-lungs" in bodies found in water? Am J Forensic Med Pathol 2004;25(4):291-301.

352. Orlowski JP, Szpilman D. Drowning: rescue, resuscitation and reanimation. Pediatr Clin North Am 2001;48:627-646.

353. Hunter TB, Whitehouse WM. Freshwater near drowning: radiological aspects. Radiology 1974;112:51-56.

354. Westacott P. A most unlikely patient. Med J Aust 1980; 2:157-158.

355. Modell JH. The effects of fluid volume in seawater drowning. Ann Intern Med 1967;67:68.

356. Modell JH, Graves SA, Ketover A. Clinical course of 91 consecutive near drowning victims. Chest 1976;10:231238.

357. Tipton, MJ. The initial responses to cold-water immersion in man. Clin Sci 1989;77:581-588.

358. Lunetta P, Penttila A, Sajantila A. Circumstances and macropathologic findings in 1590 consecutive cases of bodies found in water. Am J Forensic Med Pathol 2002;23: 371-376

359. Fuller RH. The 1962 Wellcome Prize Essay. Drowning and the postimmersion syndrome. A clinicopathologic study. Mil Med 1963;128:22-36.

360. Morild I. Pleural effusion in drowning. Am J Forensic Med Pathol 1995;16:253-256

361. Thomas F, Van Hecke W, Timperman J. The medicolegal diagnosis of death by drowning. J Forensic Sci 1963;8: $1-14$.

362. Spitz WU, Schneider V. The significance of diatoms in the diagnosis of death by drowning. J Forensic Sci 1964;9: 11-18.

363. Gregg PJ,Walder DN. Caisson disease of bone. Clin Orthop 1986;210:43-54.

364. Van Blarcom ST, Czarnecki, DJ, Fueredi, GA, Wenzel, MS. Does dysbaric osteonecrosis progress in the absence of further hyperbaric exposure? A 10-year radiologic followup of 15 patients. Am J Roentgenol 1990;155:95-97.

365. Butler FK. Decompression sickness presenting as optic neuropathy. Aviat Space Environ Med 1991;62:346-350.

366. Butler FK. Ocular manifestations of decompression sickness. In: Gold DH, Weingeist TA, eds. The eye in systemic disease. Philadelphia: Lippincott Williams \& Wilkins, 1990.

367. Kizer K. Scuba diving and dysbarism. In: Auerbach P, ed. Wilderness medicine, 3rd ed. St. Louis: Mosby, 1995: 1176-1208.

368. DeGorordo A, Vallejo-Manzur F, Chanin K, Varon J. Diving emergencies. Resuscitation 2003;59:171-180.

369. Anonymous. Divers Alert Network. Report on decompression illness and diving fatalities. Flagstaff, AZ. Best Publishing, 1998.

370. Melamed Y, Shupak A, Bitterman H. Medical problems associated with underwater diving. N Engl J Med 1992;326: 30-35.

371. Kizer KW, Goodman PG. Radiographic manifestations of venous air embolism. Radiology 1982;144:35-39.

372. Loewenherz JW. Pathophysiology and treatment of decompression sickness and gas embolism. J Fla Med Assoc 1992;79:620-624.

373. Miller K, Chang A. Acute inhalation injury. Emerg Med Clin North Am 2003;21:533-557.

374. Walker JE,Wells RE, Merrill EW. Heat and water exchange in the respiratory tract. Am J Med 1961;30:259-267.

375. Enkhbaatar P, Traber DL. Pathophysiology of acute lung injury in combined burn and smoke inhalation injury. Clin Sci 2004;107:137-143.

376. Bowen BD, Bert JL, Gu X, Lund T, Reed RK. Microvascular exchange during burn injury. Shock 1989;28:221-233.

377. Lund T, Bert JL, Onarheim H, Bowen BD, Reed RK. Microvascular exchange during burn injury. Shock 1989;28: 179-197.

378. Soejima K, Schmalstieg FC, Sakurai H, Traber LD, Traber DL. Pathophysiological analysis of combined burn and smoke inhalation injuries in sheep. Am J Physiol Lung Cell Mol Physiol 2001;280:L1233-L1241.

379. U.S. Army Medical Research Institute of Infectious Diseases. Medical management of biological casualties handbook, 4th ed. U.S. Army Medical Research Institute of Infectious Diseases, 2001.

380. Sidell FR, Takafuji ET, Franz DR. Medical aspects of chemical and biological warfare. Washington, DC: Office of the Surgeon General, 1997.

381. Gilchrist, HL. Medical aspects of gas warfare. The Medical Department of the United States Army in the World War. 1926;14:250-272.

382. Willems JL. Clinical management of mustard gas casualties. Ann Med Mil Belg 1989;3(suppl):1-6.

383. Urbanetti, JS. Battlefield chemical inhalation injury. In: Loke J, ed. Pathophysiology and treatment of inhalation injuries. New York: Marcel Dekker, 1988.

384. Balali M. Clinical and laboratory findings in Iranian fighters with chemical gas poisoning. Proceedings of the $1^{\text {st }}$ World Congress on New Compounds in Biological and Chemical Warfare: Toxicological Evaluation, May 21-23, 1984, Part III:254-259. 
385. Balali M. First report of delayed toxic effects of yperite poisoning in Iranian fighters. Proceedings of the $1^{\text {st }}$ World Congress on New Compounds in Biological and Chemical Warfare: Toxicological Evaluation, Industrial Chemical Disasters, Civil Protection and Treatment, August 24-27, 1986, Part I:489-496.

386. Freitag L, Firusian N, Stamatis G, Greschuchna D. The role of bronchoscopy in pulmonary complications due to mustard gas inhalation. Chest 1991;100:1436-1441.

387. Buscher H, Conway N. Green and yellow cross. Kettering Laboratory of Applied Physiology, University of Cincinnati, 1944.

388. Morgenstern P, Koss FR, Alexander WW. Residual mustard gas bronchitis: effects of prolonged exposure to low concentrations of mustard gas. Ann Intern Med 1947;26: 27-40.

389. McAdams AJ Jr, Joffe MH. A toxico-pathologic study of phosgene oxime. Medical Laboratories Research Report 381; Army Chemical Center, MD: 1955.

390. Franz DR, Jahrling PB, Friedlander AM, et al. Clinical recognition and management of patients exposed to biological warfare agents. JAMA 1997;278(5):399-411.

391. Lamps LW, Havens JM, Sjostedt A, Page DL, Scott MA. Histologic and molecular diagnosis of tularemia: a potential bioterrorism agent endemic to North America. Mod Pathol 2004;17:489-495.

392. Wilhelmsmen C, Pitt L. Lesions of acute inhaled lethal ricin intoxication in rhesus monkeys. Vet Pathol 1996;33: 296-302.

393. Brugsch HG. Toxic hazards: the castor bean. Mass Med Soc 1960;262:1039-1040.

394. Tomashefski JF Jr, Felo JA. The pulmonary pathology of illicit drug and substance abuse. Curr Diagn Pathol 2004; 10:413-426.

395. Karch SB. Karch's pathology of drug abuse. Boca Raton, FL: CRC Press, 2002.

396. Hall W, Hando J. Illicit amphetamine use as a public health problem in Australia. Med J Aust 1993;159(10): 643-644.

397. Tharp AM, Winecker RE, Winston DC. Fatal intravenous fentanyl abuse: four cases involving extraction of fentanyl from transdermal patches. Am J Forensic Med Pathol 2004;25(2):178-181.

398. Bezmalinovic Z, Gonzalez M, et al. Orophayngeal injury possibly due to free-base cocaine. N Engl J Med 1988; 319(21):1420-1421.

399. Snyderman C, Weissmann J, et al. Crack cocaine burns of the larynx. Arch Otolaryngol Head Neck Surg 1991;117(7): 792-795.

400. Reino AJ, Lawson W. Upper airway distress in crackcocaine users. Otolaryngol Head Neck Surg 1993;109(5): 937-940.

401. McQueen CT, Yarbroug, WG, et al. Unilateral supraglottitis in adults: fact or fiction. J Otolaryngol 1995;24(4): 255-257.

402. Moettus A, Tandberg D. Brillo pad crack screen aspiration and ingestion. J Emerg Med 1998;16(6):861-863.

403. Morris RR. Human pulmonary histopathological changes from marijuana smoking. J Forensic Sci 1985;30(2): 345-349.
404. Tennant FS Jr. Histopathologic and clinical abnormalities of the respiratory system in chronic hashish smokers. National Institute Drug Abuse Research Monogram 1980;series 27:309-315.

405. Council on Scientific Affairs. Marijuana: its health hazards and therapeutic potentials. JAMA 1981;246(16):18231827.

406. Patrick GB. Marijuana and the lung. Postgraduate Medicine 1980;67(5):110-118.

407. Tashkin DP, Calvarese MPH, Simmons MS, Shapiro BJ. Respiratory status of seventy-four habitual marijuana smokers. Chest 1980;78(5):699-706.

408. Klinger JR, Bensadown E, Corrao WM. Pulmonary complications from alveolar accumulation of carbonaceous material in a cocaine smoker. Chest 1992;101(4):1171-1173.

409. Laposata EA, Mayo GL. A review of pulmonary pathology and mechanisms associated with inhalation of freebase cocaine ("crack"). Am J Forensic Med Pathol 1993;14(1): 1-9.

410. Haim DY, Lippmann ML, Goldberg SK, Walkenstein MD. The pulmonary complications of crack cocaine. A comprehensive review. Chest 1995;107:233-240.

411. Rao AN, Polos PG, Walther FA. Crack abuse and asthma: a fatal combination. NY State J Med 1990;90:511-512.

412. Rebhun J. Association of asthma and freebase smoking. Ann Allergy 1988;60:339-342.

413. Kinmouth JB. The lymphatics: surgery, lymphography, and diseases of the chyle and lymph systems. 2nd ed. London, Arnold, 1982:252.

414. Suhl J, Gorelick DA. Pulmonary function in male freebase cocaine smokers. Am Rev Respir Dis 1988;137:A488.

415. Rubin RB, Neugarten J. Cocaine-associated asthma. Am J Med 1990;88:438-439.

416. Tashkin DP, Simmons MS, Chang P. Effects of smoked substance abuse on nonspecific airway hyperresponsiveness. Am Rev Respir Dis 1993;147:97-103.

417. Hughes S, Calvery PMA. Heroin inhalation and asthma. Br Med J 1988;297:1511-1512.

418. Kagen SL, Kurup VP, Sohnle PG, Fink JN. Marijuana smoking and fungal sensitization. J Allerg Clin Immunol 1983;71:389-393.

419. Llamas R, Hart DR, Schneider NS. Allergic bronchopulmonary aspergillosis associated with smoking moldy marijuana. Chest 1978;73:871-872.

420. Cunning D, Teichtahl $\mathrm{H}$. Necrotizing pulmonary granulomata in a marijuana smoker. Chest 2000;117:1511-1514.

421. Chusid MJ, Gelford JA, Nutter C, Fauci AS. Pulmonary aspergillosis, inhalation of contaminated marijuana smoke, chronic granulomatous disease. Ann Intern Med 1975;82: 682-683.

422. Banner AS, Rodriguez J, Sunderrajan EV. Bronchiectasis: a cause of pulmonary symptoms in heroin addicts. Respiration 1979;37:232-237.

423. Warnock ML, Ghahremani GG, Rattenborg C. Pulmonary complication of heroin intoxication. Aspiration pneumonia and diffuse bronchiectasis. JAMA 1972;219:1051-1053.

424. Tashkin DP. Pulmonary complications of smoked substance abuse. West J Med 1990;152:525-530.

425. Barsky SH, Roth MD, Kleerup EC. Histopathological and molecular alterations in bronchial epithelium in habitual 
smokers of marijuana, cocaine and tobacco. J Natl Cancer Inst 1998;90:1198-1205.

426. Duberstein JL, Kaufman DM. A clinical study of an epidemic of heroin Intoxication and heroin-induced pulmonary edema. Am J Med 1971;51:704-714.

427. Siegel H. Human pulmonary pathology associated with narcotic and other addictive drugs. Hum Pathol 1972; 3:55-66.

428. Byers JM, Soin JS, Fisher RS, Hutchins GM. Acute pulmonary alveolitis in narcotics abuse. Arch Pathol 1975;99: 273-277.

429. Cucco RA, Yoo OH, Cregler L, Chang JC. Nonfatal pulmonary edema after "freebase" cocaine smoking. Am Rev Respir Dis 1987;136:179-181.

430. Murray RJ, Albin RJ, Mergner W, Criner GJ. Diffuse alveolar hemorrhage temporarily related to cocaine smoking. Chest 1988;93:427-429.

431. Bailey ME, Fraire AE, Greenberg SD. Pulmonary histopathology in cocaine abusers. Hum Pathol 1994;25: 203-207.

432. Heffner JE, Harley RA, Schabel S. Pulmonary reactions from illicit substance abuse. Clin Chest Med 1990;11: 151-162.

433. Godwin JE, Harley RA, Miller KS, Heffner JE. Cocaine, pulmonary hemorrhage, and hemoptysis. Ann Intern Med 1989;110:843.

434. Baldwin GC, Buckley DM, Roth MD. Acute activation of circulating polymorphonuclear neutrophils following in vivo administration of cocaine. A potential etiology for pulmonary injury. Chest 1997;111:698-705.

435. Kissner DG, Lawrence WD, Selis JE, Flint A. Crack lung: pulmonary disease caused by cocaine abuse. Am Rev Respir Dis 1987;136:1250-1252.

436. Forrester JM, Steele AW, Waldron JA, Parsons PE. Crack lung: an acute pulmonary syndrome with a spectrum of clinical and histopathologic findings. Am Rev Respir Dis 1990;142:462-467.

437. Patel RC, Dutta D, Schonfeld SA. Freebase cocaine use associated with bronchiolitis obliterans organizing pneumonia. Ann Intern Med 1987;107:186-187.

438. Kringholm B, Christoffersen P. The nature and the occurrence of birefringent material in different organs in fatal drug addiction. Forensic Sci Int 1987;34:53-62.
439. Ganesan S, Felo J, Saldana M, et al. Embolized crospovidone(poly(N-vinyl-2-pyrrolidone)) in the lungs of intravenous drug users. Mod Pathol 2003;16:286292.

440. DeRuggiere MA, Pampiglione E, et al. A case of embolism caused by metallic mercury in a drug addict. Ann Ig 1989; 1(3-4):673-678.

441. Haffner HT, Erdelkamp J, et al. Morphological and toxicological findings after intravenous injection of metallic mercury. Dtsch Med Wochenschr 1991;116(36):1342-1346.

442. Abraham J, Brambilla C. Particle size for differentiation between inhalation and injection pulmonary talcosis. Env Res 1980;21:94-96.

443. Murray RJ, Smialek JE, Golle M, Albin RJ. Pulmonary artery medial hypertrophy in cocaine users without foreign particle microembolization. Chest 1989;96:1050-1053.

444. Abenhaim L, Moride Y, Brenot F, et al. Appetite-suppressant drugs and the risk of primary pulmonary hypertension. N Engl J Med 1996;335(9):609-616.

445. Schaiberger PH, Kennedy TD, Miller FC. Pulmonary hypertension associated with long-term inhalation of 'crank' methamphetamine. Chest 1993;104:614-616.

446. Shesser R, Davis C, Edelstein S. Pneumomediastinum and pneumothorax after inhaling alkaloidal cocaine. Ann Emerg Med 1981;10:213-215.

447. Adrouny A, Magnusson P. Pneumopericardium from cocaine inhalation. N Engl J Med 1985;313:48-49.

448. Miller WE, Spiekerman RE, Hepper NC. Pneumomediastinum resulting from performing Valsalva maneuvers during marijuana smoking. Chest 1972;62:233-234.

449. Johnson MK, Smith RP, Morrison D. Large lung bullae in marijuana smokers. Thorax 2000;55:340-342.

450. Van de Klooster JM, Grootendorst AF. Severe bullous emphysema associated with cocaine smoking. Thorax 2001;56:982-983.

451. Goldstein DS, Karpel JP, Appel D, Williams MH. Bullous pulmonary damage in users of intravenous drugs. Chest 1986;89:266-269.

452. Forensic Pathology Committee College of American Pathologists. The pathologist in court. College of American Pathologists, 2003.

453. Davis GG. Pathology and law: a practical guide for the pathologist. New York: Springer-Verlag, 2004. 